The psychophysiology primer : A guide to methods and a broad review with a focus on human-computer interaction

\title{
Cowley, Benjamin
}

2016

Cowley , B , Filetti , M , Lukander , K, Torniainen , J, Henelius , A, Ahonen , L, Barral , O , Kosunen , I, Valtonen , T, Huotilainen, M , Ravaja, N \& Jacucci , G 2016 , ' The psychophysiology primer : A guide to methods and a broad review with a focus on human-computer interaction ', Foundations and Trends in Human-Computer Interaction , vol. 9 , no. 3-4 , pp. 151-308 . https://doi.org/10.1561/1100000065

http://hdl.handle.net/10138/172968

https://doi.org/10.1561/1100000065

other

acceptedVersion

Downloaded from Helda, University of Helsinki institutional repository.

This is an electronic reprint of the original article.

This reprint may differ from the original in pagination and typographic detail.

Please cite the original version. 
Foundations and Trends ${ }^{\circledR}$ in Human-Computer Interaction

Vol. 9, No. 3-4 (2015) 151-308

(C) 2016 B. Cowley, M. Filetti, K. Lukander,

J. Torniainen, A. Henelius, L. Ahonen, O. Barral,

I. Kosunen, T. Valtonen, M. Huotilainen,

N. Ravaja, G. Jacucci

DOI: $10.1561 / 1100000065$ now

the essence of knowledge

\title{
The Psychophysiology Primer: A Guide to
} Methods and a Broad Review with a Focus on Human-Computer Interaction

\author{
Benjamin Cowley ${ }^{1,2}$, Marco Filetti ${ }^{3,4}$, Kristian Lukander ${ }^{1}$, \\ Jari Torniainen ${ }^{1}$, Andreas Henelius ${ }^{1}$, Lauri Ahonen ${ }^{1}$, \\ Oswald Barral $^{4}$, Ilkka Kosunen ${ }^{4}$, Teppo Valtonen ${ }^{1}$, \\ Minna Huotilainen ${ }^{7}$, Niklas Ravaja ${ }^{3,5,6}$, and Giulio Jacucci ${ }^{3,4}$ \\ 1. Quantified Employee Unit, Finnish Institute of Occupational Health, \\ Helsinki, Finland \\ 2. Cognitive Brain Research Unit, Institute of Behavioral Sciences, \\ University of Helsinki, Helsinki, Finland \\ 3. Helsinki Institute for Information Technology HIIT, \\ Department of Computer Science, Aalto University, Espoo, Finland \\ 4. Helsinki Institute for Information Technology HIIT, \\ Department of Computer Science, University of Helsinki, \\ Helsinki, Finland \\ 5. Helsinki Collegium for Advanced Studies, University of Helsinki, \\ Helsinki, Finland \\ 6. Finland School of Business, Aalto University, Helsinki, Finland \\ 7. Finnish Institute of Occupational Health, Helsinki, Finland
}




\section{Contents}

1 Introduction 152

1.1 Related reviews . . . . . . . . . . . . . . 155

2 Definitions 157

3 The state of the art $\quad 166$

3.1 Cardiovascular signals . . . . . . . . . . . . . . . 168

3.2 Electrodermal activity . . . . . . . . . . . . . . . 176

3.3 Respiration . . . . . . . . . . . . . . . . . . . . 186

3.4 Electromyography . . . . . . . . . . . . . . . . . . . 191

3.5 Oscillatory electroencephalography . . . . . . . . . . 197

3.6 Event-related brain potentials . . . . . . . . . . . . . . 207

3.7 Pupillometry . . . . . . . . . . . . . . . . . . . . . . . . . . . 214

3.8 Eye tracking . . . . . . . . . . . . . . . . . . . . . 218

3.9 Video. . . . . . . . . . . . . . . . . . . . . . . . . 223

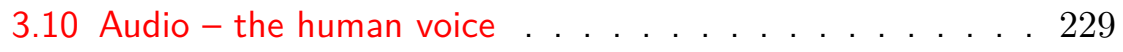

3.11 Multimodal signal classification . . . . . . . . . . . . 233

3.12 Solutions for online data processing and fusion . . . . . . . 241

4 Overview and application $\quad 249$

4.1 Application areas . . . . . . . . . . . . . . . . . 259

4.2 Practical guidelines . . . . . . . . . . . . . . . . . 262 
5 Concluding remarks $\quad 267$

$\begin{array}{ll}\text { Acknowledgements } & 271\end{array}$

$\begin{array}{ll}\text { References } & 272\end{array}$ 


\section{Abstract}

Digital monitoring of physiological signals can allow computer systems to adapt unobtrusively to users, so as to enhance personalised 'smart' interactions. In recent years, physiological computing has grown as a research field, and it is increasingly considered in diverse applications, ranging from specialised work contexts to consumer electronics. Working in this emerging field requires comprehension of several physiological signals, psychophysiological states or 'indices', and analysis techniques. The resulting literature encompasses a complex array of knowledge and techniques, presenting a clear challenge to the practitioner.

We provide a foundational review of the field of psychophysiology to serve as a primer for the novice, enabling rapid familiarisation with the core concepts, or as a quick-reference resource for advanced readers. We place special emphasis on everyday human-computer interface applications, drawing a distinction from clinical or sports applications, which are more commonplace. The review provides a framework of commonly understood terms associated with experiential constructs and physiological signals. Then, 12 short and precisely focused review chapters describe 10 individual signals or signal sources and present two technical discussions of online data fusion and processing. A systematic review of multimodal studies is provided in the form of a reference table. We conclude with a general discussion of the application of psychophysiology to human-computer interaction, including guidelines and challenges.

B. Cowley, M. Filetti, K. Lukander, J. Torniainen, A. Henelius, L. Ahonen, O. Barral, I. Kosunen, T. Valtonen, M. Huotilainen, N. Ravaja, G. Jacucci. The Psychophysiology Primer: A Guide to Methods and a Broad Review with a Focus on Human-Computer Interaction. Foundations and Trends ${ }^{\circledR}$ in Human-Computer Interaction, vol. 9, no. 3-4, pp. 151-308, 2015.

DOI: $10.1561 / 1100000065$. 
The psychophysiological method uses measurements of physiology to form inferences about states of mind. The aim is to extract quantitative indices of essentially qualitative cognitive or affective states. Because the method does not impose restrictions on the physiological signals that are measurement sources, it has an extensive area of possible focus. Since the measurements are quantitative while cognitive and affective states are qualitative, there is an issue of establishing ground truth, and the choice of psychological model becomes important. Clearly, there are enormous challenges. Among the ways of addressing these challenges are accounting for the context of the individual during the recording and using multimodal data. However, the primary requirement is an effective working knowledge of the range of relevant signals and their application.

Human-computer interaction (HCI) is one domain wherein the psychophysiological method can be efficiently applied. In this application, human-facing software accesses psychophysiological indices from its user(s) in order to adapt via some internal logic and, for example, alter the information display so as to enhance the cognitive ergonomics. The vision is that, in conjunction with 'smart' software, the human 


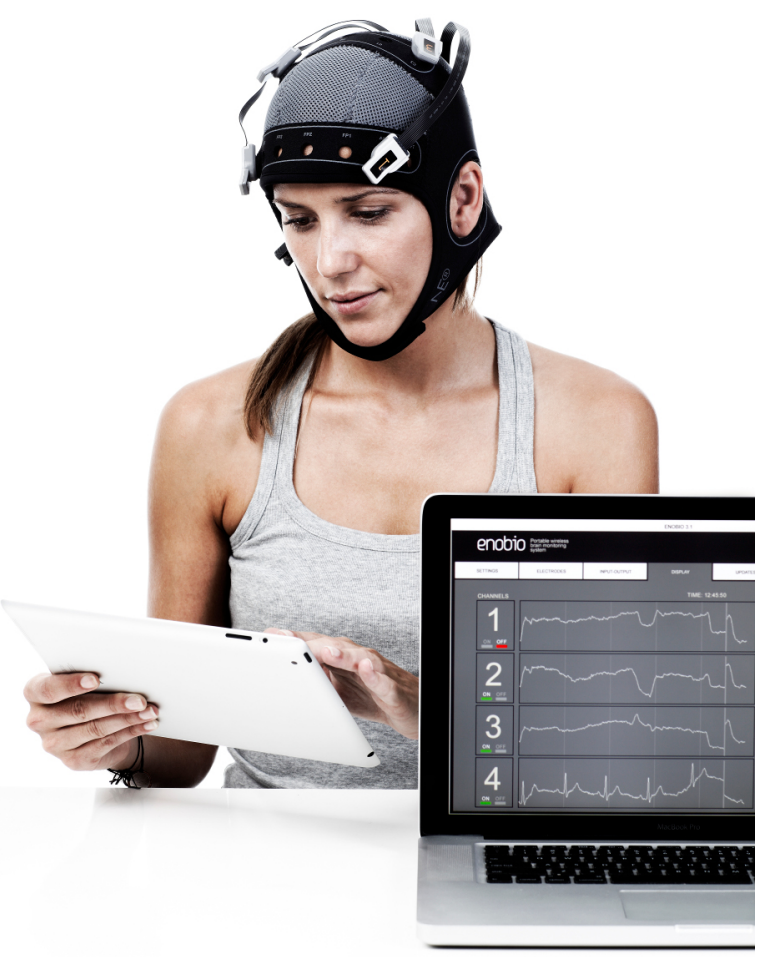

Figure 1.1: An example of an ambulatory psychophysiological set-up with an EEG amplifier plus mobile and fixed devices. Image reproduced with permission from Neuroelectrics SLU (Barcelona, Spain).

user performs the tasks that humans carry out best and is aided by software that automates other types of tasks.

As in other psychophysiology applications, such as clinical or sports uses, it is important to link the task and environment context with physiology signals such that user-facing systems, systems in the environment, and sensors all contribute to the same end, as illustrated in Figure 1.1. 
We present a reference guide and primer to the concepts and methods of psychophysiology, along with its uses in HCI, thereby enabling rapid familiarisation with the core concepts across a broad swath of the field of psychophysiology in HCI. Although this field is very broad, taking an essentially practical approach enables us to present a relatively comprehensive overview of the relevant topics.

Our focus constrains the psychophysiological technology that we consider here, as we are concerned with only those devices that are lightweight, wearable or remotely operable, and application-ready. This rules out room-sized sensors, such as the ones utilised in functional magnetic resonance imaging.

Overview Section 1.1 briefly describes prior reviews of psychophysiology in HCI, after which the following organisation is used:

- 2: Definitions: the terminology necessary for understanding signals and associated metrics with which one may index psychological states

- 3: The state of the art: description of each of the most commonly used signals or methods, in turn, in sections 3.1 to 3.10, then sections 3.11 and 3.12, which focus on signal fusion, from both a theoretical and a practical perspective

- 4: An overview and reference to guide users of psychophysiology, including a reference table summarising prior work, along with sections on generic application areas for psychophysiology in HCI (4.1) and general guidelines for use, which also serve as a practical reference for putting the information in this primer to use (4.2)

- 5: Concluding remarks on the complexities and limitations of psychophysiology, with an introduction to the subtle challenges posed by more complicated issues of theory and epistemology, such as choice of interpretive model 


\subsection{Related reviews}

The foundational text for the field of psychophysiology is arguably the Handbook of Psychophysiology (Cacioppo et al., 2000), a comprehensive reference covering the underlying scientific disciplines. With more than 1,000 pages, this comprehensive handbook cements many principles and covers a wide range of subjects, such as developmental, clinical, and environmental psychophysiology. The range of topics extends well beyond HCI, in fact, hence falling beyond the scope of our interest.

A reference book with a more applied orientation is Engineering Psychophysiology (Backs and Boucsein, 2000), which proceeds from engineering psychology and ergonomics. Research in these fields can be considered to be closer to our scope. The first part of the book reviews issues such as methodological considerations, theoretical issues, signal processing, and recording methods, whilst the application part reports on a mixture of laboratory and realistic studies, thereby demonstrating a relevant gap to the application of psychophysiology in the real world.

In HCI, psychophysiology has been gaining ground as both a method for studying user experience and a technique to be incorporated into interactive systems. Particularly instrumental for the latter has been the introduction of physiological computing as a field in which human physiological data act as system input in real time (Allanson and Fairclough, 2004). Early reviews of physiological computing highlighted the complexity of psychophysiological inference and its validation (Park, 2009), alongside challenges in representing the psychophysiological state of the user (Dirican and Göktürk, 2011), designing explicit and implicit interventions in the bio-cybernetic loop, and ethics implications (Fairclough, 2009b). Recently, several books (Fairclough and Gilleade, 2014) and special issues of journals (Silva et al., 2015; Jacucci et al., 2015) have reported on advances in physiological computing for HCI.

Several reviews have covered some portion of the research corpus on the application of psychophysiology in HCI. Psychophysiology, along with multimodal techniques, has been gaining attention in the field of Affective Computing, as evidenced in recent reviews (D'mello and Kory, 2015; Lopatovska and Arapakis, 2011; D’Mello and Kory, 2012; Zeng 
et al., 2009). Usually these are high-level reviews considering how fusing and combining modalities is possible in detection of emotions, and they seldom go into the details of each signal or metric (Calvo and D'Mello, 2010). Ravaja (2004) described the literature on the application of psychophysiology in Media Psychology research, while Kivikangas et al. (2011) created a review for computer-game research.

Furthermore, specific topics have inspired recent reviews. Cowley (2015), for instance, undertook a preliminary review of the use of psychophysiology to assess states of high-level cognition, a new and promising area of study, which is typically associated with decision-making, problem-solving, and executive control of cognition and action. Novak et al. (2012) compiled a detailed review of data-fusion applications (the source for Tables 2.1, 2.2, and 4.1). Other, partially overlapping reviews include those by Lisetti and Nasoz (2004) and by Jerritta et al. (2011), which briefly summarise previous applications in emotion recognition and affective computing. Another high-level review, by Brouwer et al. (2015), focuses on the most successful applications, warning about some pitfalls and providing recommendations.

In light of the above, there is a clear absence of a reference article geared for HCI that could serve as a compact guide for researchers of various backgrounds. 


\section{2}

\section{Definitions}

This chapter defines psychological and biological terms, signals, and associated quantitative metrics. The terms listed below are defined for the scope of this review alone - i.e., they apply to human psychophysiology in computer interactions ${ }^{1}$. Section 3.12 provides a more detailed picture of signal processing concepts.

- Sensor: a physical device for the measurement of signals from physical systems - in our case, people. Examples are electrodes, cameras, and accelerometers.

- Signal: a real-time data stream supplied by a sensor.

- Cognitive/affective state: any interesting aspect of the user's state that can be interpreted from the user's physiology and thus measured by sensors (e.g., emotion or cognition).

\footnotetext{
${ }^{1}$ Our terms are intended to follow the literature as closely as possible, but variation across sources renders it impossible to offer definitions that are both unique and universal. For the interested reader, some reference texts (Cacioppo et al., 2000) are more foundational than others.
} 
- Metric: a quantitative feature of a signal, such as heart rate derived from a cardiogram by measurement of the number of heart beats (R-peaks) within a given time window.

- Index (pl. indices): a scalar representing a cognitive state, built from one or more metrics. An index is, in essence, a model of the user's cognitive/affective state, so the usual issues that accompany modelling, such as ground truth and goodness of fit, are pertinent.

- Client: any external application that accesses the psychophysiology data after the primary processing is carried out.

- Processing element: an atomic unit utilised for performing computations on the incoming signal within a system.

- Classifier: a statistical algorithm that separates the multidimensional space of one or more metrics into two or more regions, corresponding to an index or indices. For psychophysiology, a classifier is usually supervised; it is trained on data pre-labelled with indices from some model - for instance, the valence/arousal emotional circumplex (see Figure 2.1).

- System: an integrated set of hardware and software employed to manage one or more sensors, derive metrics from signals, and transform metrics to indices for transmission to clients. Details on technical aspects are given in section 3.12, below.

The next step is to define the signals covered in Table 2.1 and the metrics in Table 2.2, which are reviewed in the sections below on the state of the art. After chapter 3 is an overview of the indices. Provided in Table 4.1, this serves as a ready reference. It should be borne in mind that the signals described in Table 2.1 can be used either to infer one or more psychological indices or for using the signals for control or feedback, as discussed in section 4.1. That discussion is followed by articulation of a general approach to selecting signals and metrics when one is starting to work in the realm of psychophysiology, in section 4.2.

Features extracted from the signals determine the inferences that one can draw about the underlying psychological process; therefore, 


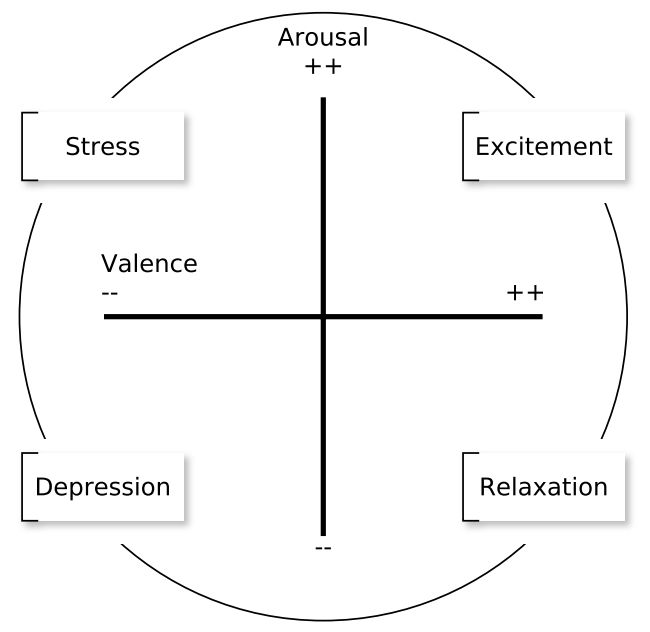

Figure 2.1: The simple emotional circumplex model, with orthogonal bipolar dimensions of arousal (from alert to lethargic) and valence (pleasant to unpleasant).

the choice of features is highly important. Feature extraction is a rich topic, largely beyond the scope of this paper, but a brief indication of feature classes is given in Table 2.2. In the text and table, we refer only to metrics, because a) we thereby represent only features that are canonical in the literature (i.e., are associated with specific states of the individual and hence have a well-defined interpretation) and, b) technically, a feature is a single, strictly defined calculation formula, whereas we deal with classes of features, in which many possible formulae can correspond to a single metric (e.g., heart rate variability can refer to the standard deviation of normal peaks (SDNN) or to the root mean square of successive differences (rMSSD)).

The review method The following method was applied for creation of these tables. We searched for recent papers surveying the literature on cognitive- and affective-state detection via psychophysiology, and then 
we selected two works that listed previous literature in a schematic and concise manner (Novak et al., 2012; Jerritta et al., 2011). The review by Novak et al. (2012) was found to cover all the indices mentioned by Jerritta et al. (2011); accordingly, all papers mentioned in the review section of Novak et al. (2012)'s work were considered. Via University of Helsinki library resources, 47 of the 54 relevant papers were accessible and downloaded. To create Tables 2.1 and 2.2, we noted, for each paper,

- which metrics were used for the 'final' classification ${ }^{2}$ and

- from which signal each metric was computed.

This systematically derived 'scoping review' provided the seed for the rest of the process. From the complete set of signals yielded by the scoping review, we selected a subset in line with the constraints of our motivating application. Firstly, we chose only those signals that either already are or should in the foreseeable future be recordable with lightweight off-the-shelf sensors appropriate for real-world HCI applications. Secondly, the signals chosen were known to be tried and tested across a substantial body of literature, and, in consequence, they are 'practical' choices for the novice and advanced user alike.

This subset is reviewed in the first 10 sections on state-of-the-art uses, below, and its elements are listed in Tables 2.1 and 2.2, above the dashed horizontal line. Below that line, in brackets, are signals that have been used in an HCI setting at least once but either are not biological in nature or lack sufficient support in the literature to merit a separate section. As with signals, metrics that were not widely used in HCI (and are not discussed in detail in this paper) are listed toward the bottom of the metric table (again, in brackets). These outof-scope signals and metrics are included for completeness, and hence are cross-referenced in Table 4.1 for all mentions in the literature.

Finally, Table 4.1 groups all indices listed in the 47 papers into categories on the basis of the exact word used to define the respective index (e.g., 'stress'). Items that were denoted with different words but

\footnotetext{
${ }^{2}$ Metrics were denoted on the basis of the procedure followed to compute them (e.g., if the metric was called 'GSR' but was recorded via the conductance method and computed for the phasic component, we labelled it 'SCR').
} 
based on very similar phenomena are placed in the same group - i.e., considered the same index (e.g., papers that indexed 'Cognitive effort' or 'Mental workload' are listed under 'Cognitive load'). Indices are cross-referenced with the section(s) in which they are discussed, and key reference citations are given for each index.

Table 2.1: List of signals in order of appearance in chapter 3

Signals not reviewed are in brackets; some are mainly behavioural in nature and hence out of scope. Those below the dashed line are used mostly in the medical domain and have poor usability for HCI.

\begin{tabular}{|c|c|c|}
\hline Signal & Description & Section \\
\hline ECG & Electrocardiogram & 3.1 \\
\hline PPG & Photoplethysmography & $3.1,3.9$ \\
\hline EDA & $\begin{array}{l}\text { Electrodermal Activity (also known as } \\
\text { Skin Conductance or Galvanic Skin } \\
\text { Response) }\end{array}$ & 3.2 \\
\hline RESP & Respiration (e.g., chest plethysmography) & $3.1,3.3,3.9$ \\
\hline EMG & Electromyography & 3.4 \\
\hline EEG & $\begin{array}{l}\text { Electroencephalography, also the source } \\
\text { of event-related potentials (ERPs) }\end{array}$ & $3.5,3.6$ \\
\hline VOG & Video-oculography (eye tracking) & $3.7,3.8$ \\
\hline EOG & Electro-oculography & 3.8 \\
\hline VID & Video-based posture/facial recognition & 3.9 \\
\hline SPEECH & Speech recorded via microphone(s) & 3.10 \\
\hline$(\mathrm{ACL})$ & Accelerometer(s) & \\
\hline (BEHAV) & $\begin{array}{l}\text { Behavioural data collected from device } \\
\text { usage (e.g., mouse movements or driving } \\
\text { controls) }\end{array}$ & \\
\hline$(\mathrm{TOT})$ & Time-on-task & \\
\hline$\overline{(\mathrm{CO} 2} \overline{)^{\prime}}$ & $\bar{C}^{-} \overline{a p n o g}-\overline{-} \bar{p} \bar{h}{ }^{-}$ & \\
\hline (HSOUND) & Heart sound & \\
\hline (ICG) & Impedance cardiogram & \\
\hline (PRS) & Pressure (e.g., via piezoelectric sensors) & \\
\hline$(\mathrm{SMM})$ & $\begin{array}{l}\text { Sphygmomanometer (incl. proprietary } \\
\text { tech such as Vasotrac) }\end{array}$ & \\
\hline$(\mathrm{TEMP})$ & Thermometer (e.g., on finger or arm) & \\
\hline
\end{tabular}




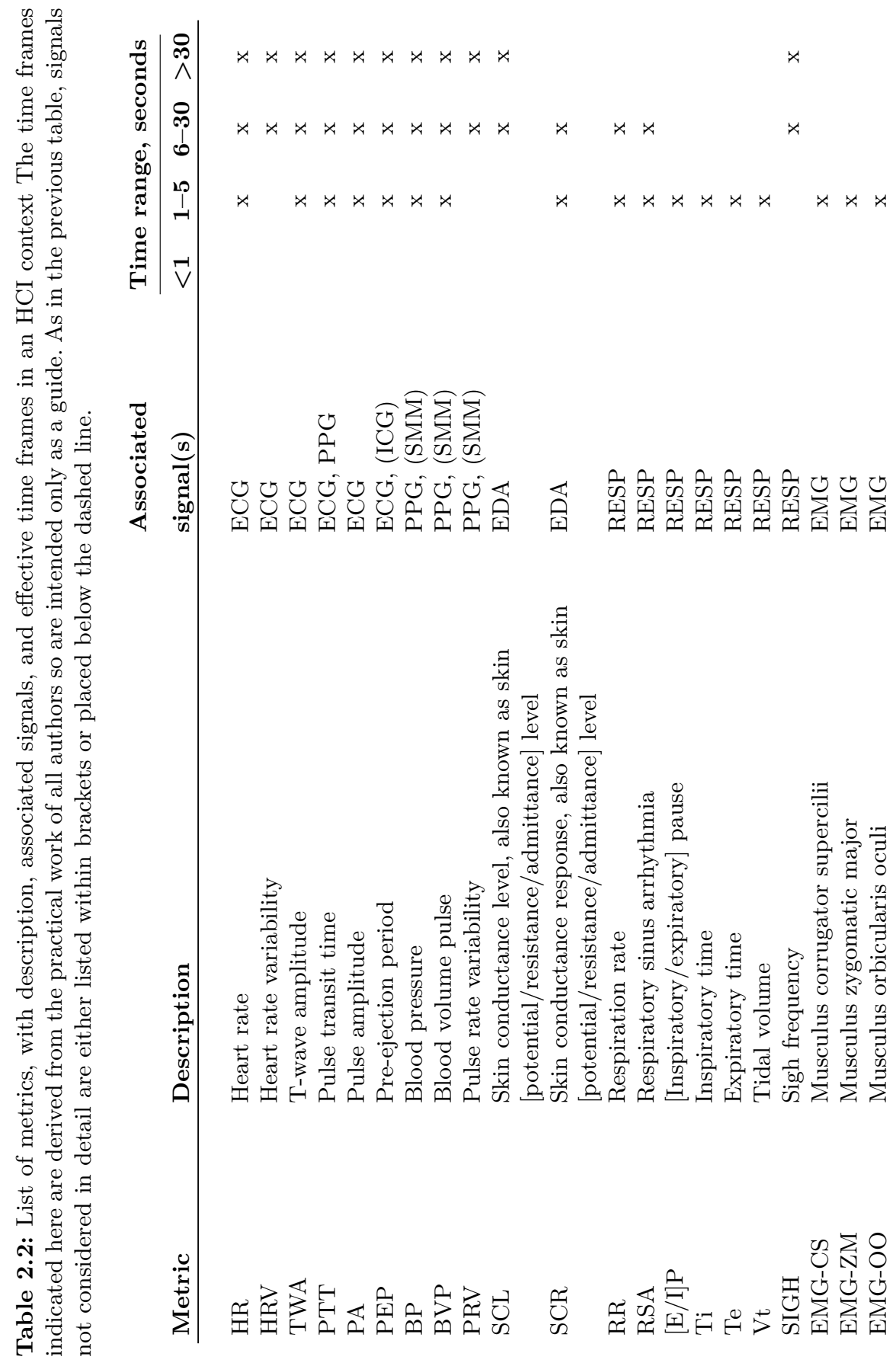




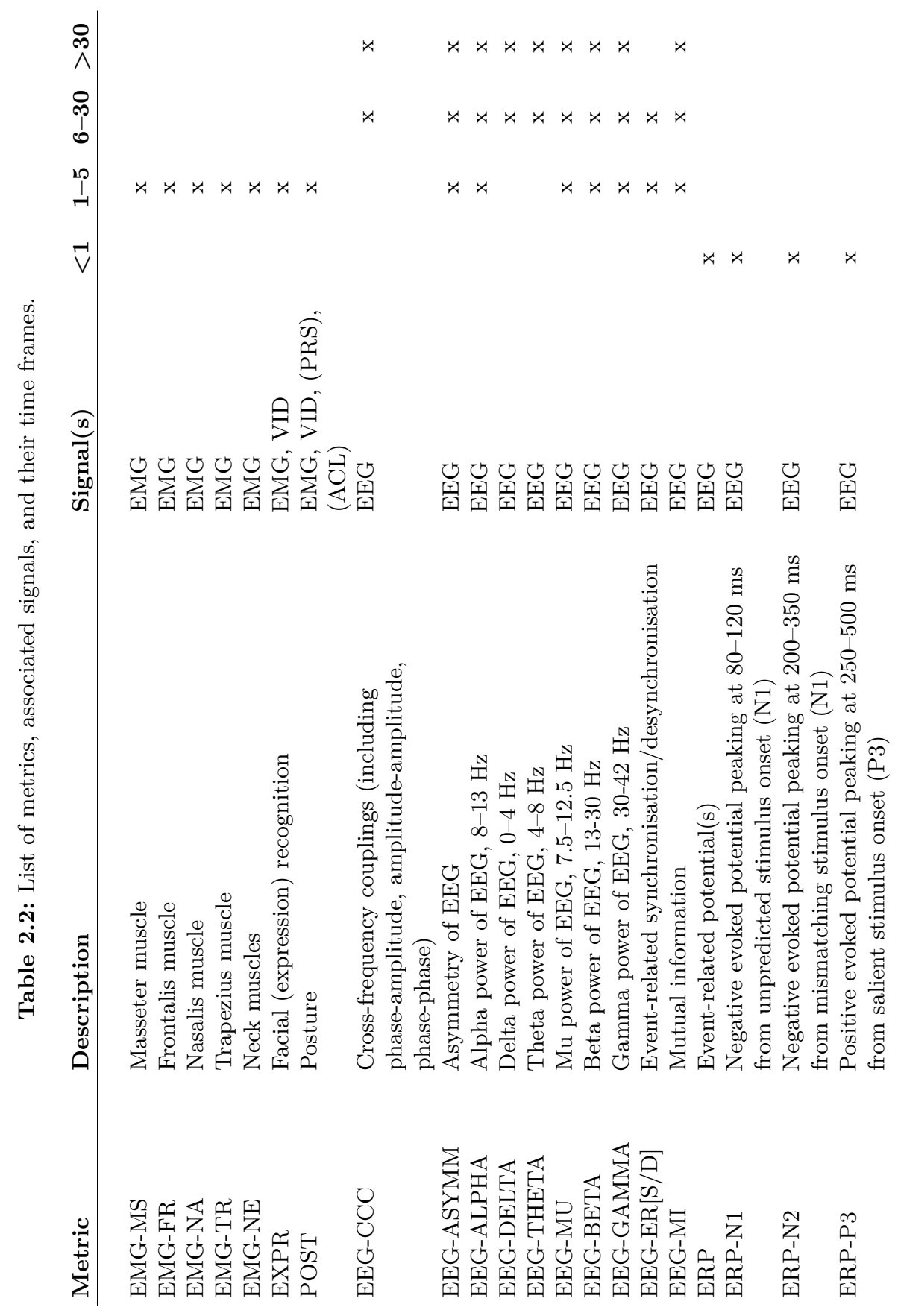




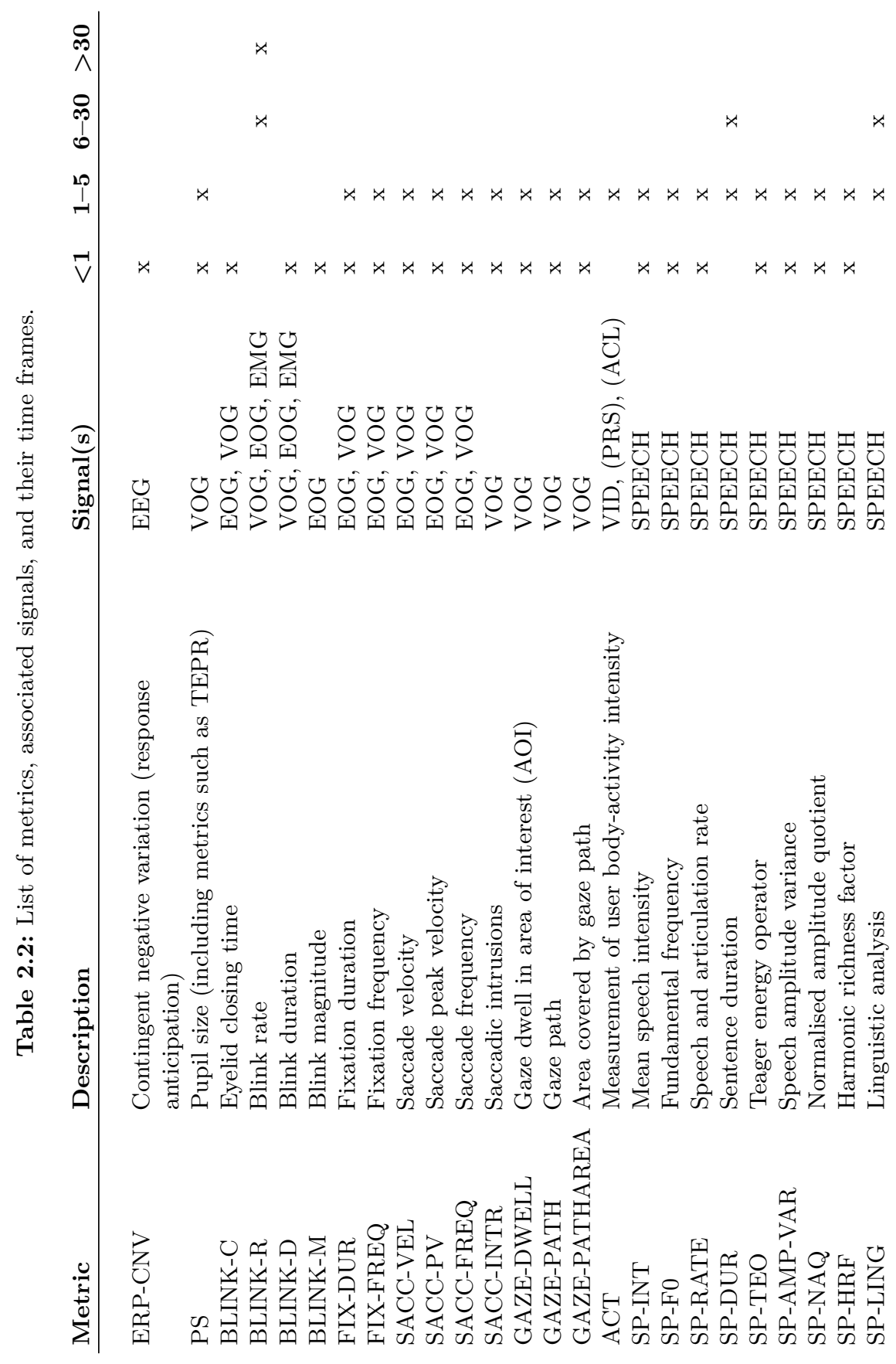




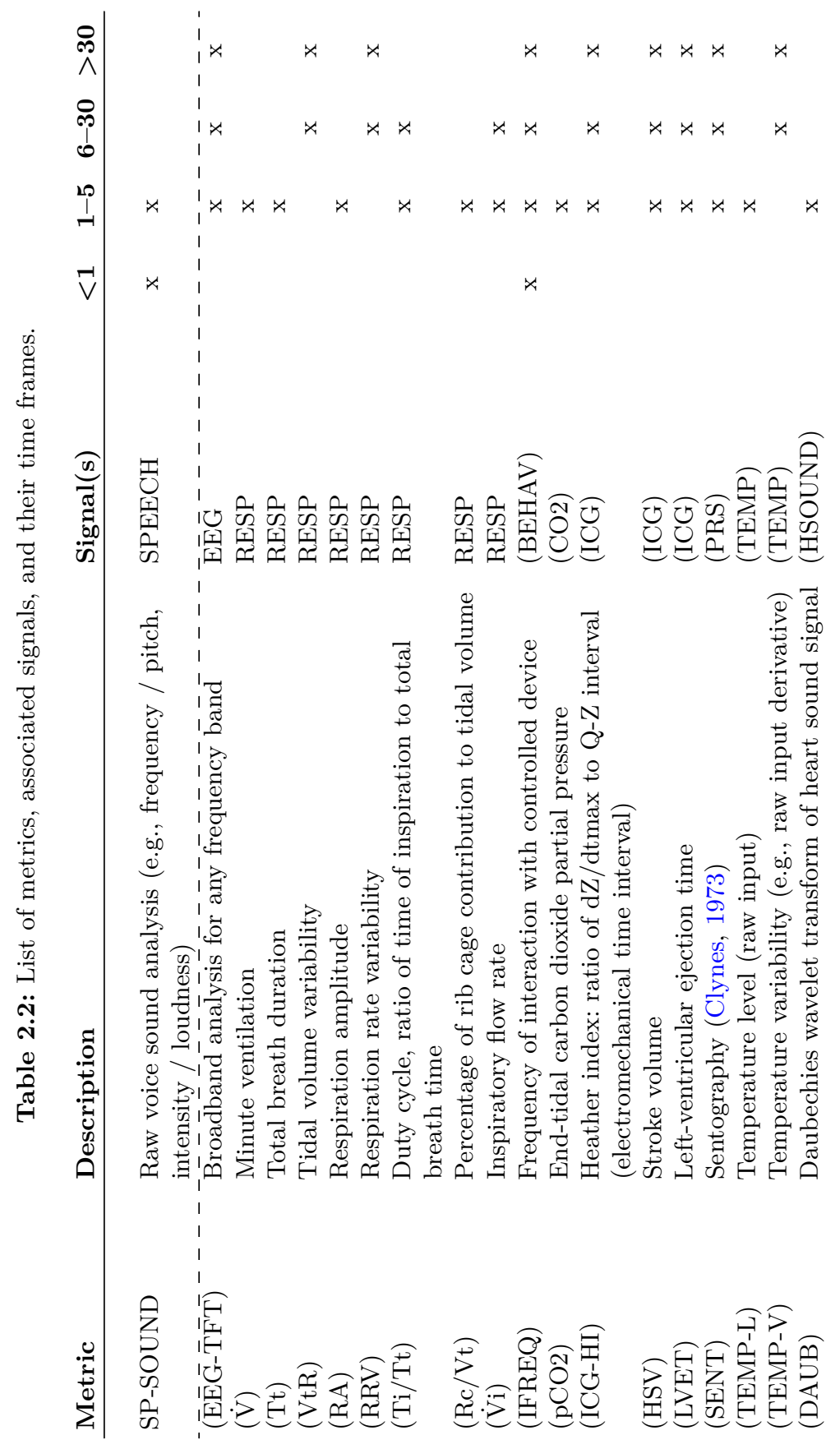




\section{3}

\section{The state of the art}

The discussion of the state of the art is arranged on the basis of three themes: internal signals ${ }^{1}$, including those from the autonomic nervous system (ANS) and the central nervous system (CNS); external signals ${ }^{2}$, encompassing the ocular system and remote recording of video and audio; and combined signals, including multimodal measurement and online processing of multiple signals. This thematic structure presents the signals roughly in order by increasing complexity of the topic, from the perspective of a novice to the field ${ }^{3}$. For internal signals, the order is approximately in line with the spectrum of signal sources from autonomic to more volitional, with most signals obtained by amplification from skin-contact electrodes. External signals, though promising, are, in general, slightly less mature or field-ready than their internal counterparts, and signal recording technologies show mixed results. The complete list of topics dealt with is as follows:

\footnotetext{
${ }^{1}$ Defined as circumspect signals traditionally recorded with skin-contact sensors.

${ }^{2}$ Defined as remotely detectable signals recorded with non-contact sensors.

${ }^{3}$ Complexity of the topic is a matter distinct from the complexity of the physiological signal source; for a review of physiology, see the Handbook of Psychophysiology (Cacioppo et al., 2000).
} 


\section{Internal signals}

\section{Autonomic nervous system}

- 3.1: Cardiovascular system

- 3.2: Skin conductance

- 3.3: Respiration

- 3.4: Electromyography

\section{Central nervous system}

- 3.5: Oscillatory electroencephalography

- 3.6: Event-related electroencephalography

\section{External signals}

\section{Ocular system}

- 3.7: Pupillometry

- 3.8: Eye tracking

\section{Remote signals}

- 3.9: Video

- 3.10: Audio (voice)

\section{Combined signals}

- 3.11: Multimodal signal classification

- 3.12: Online processing of multiple signal sources

The aim with this paper is not to describe technical requirements of the psychophysiological method, such as signal processing concerns, selection of sensor equipment, or machine learning for inference-building. However, for completeness, brief reference to works on these topics is provided in each section. 


\subsection{Cardiovascular signals}

The heart is innervated by both the sympathetic and the parasympathetic branch of the ANS. The sympathetic branch, tied to stress 'fightor-flight' responses, tends to increase heart rate, whereas parasympathetic activity, representing 'rest-and-digest' behaviour, decreases it. The rate at which the heart beats and variations thereof hence reflect the activity of the ANS. Accordingly, various metrics describing the ANS activity can be derived from cardiovascular signals. For instance, heart rate variability metrics derived from the electrocardiogram (ECG) are widely used in psychophysiology (Malik et al., 1996) - for example, to investigate phenomena such as mental workload. The cardiovascular system responds to sympathetic and parasympathetic activation within a few seconds (Berntson, 1997). However, cardiovascular metrics used in psychophysiology are typically analysed on a time scale of minutes (Malik et al., 1996) in the case of short-term heart rate variability metrics. For instance, the Trier Social Stress Test (Kirschbaum et al., 1993) has been used extensively to induce psychosocial stress, and cardiovascular and endocrine responses are well documented (Kudielka et al., 2007). In this connection, one can gain insight from a study by Lackner et al. (2010) investigating the time course of cardiovascular responses in relation to mental stress and orthostatic challenge in the form of passive head tilt-up. There are also longer rhythms evident in cardiovascular metrics, such as circadian patterns in heart rate variability (Huikuri et al., 1994).

The cardiovascular measurement techniques considered here are (i) electrocardiography, (ii) blood pressure measurement, and (iii) photoplethysmography; however, the activity of the heart can also be measured by means of various other techniques, such as ballistocardiography (Lim et al., 2015) or Doppler (Lin et al., 1979).

\section{Measurement of cardiovascular signals}

Cardiovascular signals can be measured continuously via non-invasive techniques and have been widely utilised in the measurement of mental workload (Aasman et al., 1987). The ECG represents the electrical 
activity of the heart, and the measurement is carried out with chest electrodes (Malmivuo and Plonsey, 1995). From the ECG it is possible to extract several signals, among them heart rate, or HR (denoting the absolute pace at which the heart beats) and heart rate variability (HRV), which is an umbrella concept for all metrics describing how the rhythm of the heart varies. To record HR and HRV, it is sufficient to record one lead, as only the R-peaks of the ECG waveform are required; for details, refer to Berntson (1997). For instance, affordable sports watches can be used for obtaining a signal suitable for HRV analysis (Gamelin et al., 2006).

Measuring continuous arterial blood pressure (BP) is technically more demanding than ECG measurement and requires more advanced equipment. In addition, long-term measurement of BP is not as unobtrusive as corresponding ECG measurement. Continuous BP can be measured from the finger, via the method of Peñáz (Peñáz, 1973) as implemented in, for example, the Finapres device (Wesseling, 1990) and its ambulatory version, the Portapres.

One can obtain a photoplethysmogram (PPG) either by using methods that require skin contact or remotely. See, for example, Allen (2007b) for a review on the measurement of PPG. In transmission PPG, the tissue is between the light source and the receiver, whereas in reflective PPG the light source and the receiver are next to each other on the surface of the skin, with the light only bouncing through the tissue from the source to the receiver. The PPG is typically obtained from the finger and the pinna by transmission and from the wrist via reflection. The PPG measurement can be performed remotely without skin contact by using imaging techniques to consider changes in the pulse (Sun et al., 2012c) (imaging techniques are discussed in section 3.9). Remote PPG has been used to study, for example, vasoconstrictive responses during mental workload by means of an ordinary webcam (Bousefsaf et al., 2014). Work related to this, by Vassend and Knardahl (2005), has used laser Doppler flowmetry to investigate facial blood flow during cognitive tasks.

The plethysmographic pulse amplitude (PA) depends on the degree of vasoconstriction, which, in turn, is affected by cognitive load (Iani 
et al., 2004). The pulse transit time (PTT) in the PPG has been found to be correlated with BP (Ma and Zhang, 2005; He et al., 2013). It should be noted that vasoconstrictive effects are not visible in the ear (Awad et al., 2001). The use of reflective PPG has become popular in several consumer sports watches, such as the A360 from Polar Electro (Kempele, Finland); Forerunner 225 from Garmin Ltd (Schaffhausen, Switzerland); Charge from Fitbit, Inc. (San Francisco, CA, USA); Apple Watch from Apple, Inc. (Cupertino, CA, USA); and Microsoft Band from Microsoft, Inc. (Redmond, WA, USA). Reflective PPG is used also in research equipment such as the E4 from Empatica Inc. or various sensors from Shimmer Sensing (Dublin, Ireland).

\section{Methods}

Analysis of cardiovascular signals In using the cardiovascular signals to investigate the activity of the autonomic nervous system, it is the variability of the signal that is of interest. The cardiovascular signals are hence analysed on a beat-by-beat basis. Raw, continuous cardiovascular signals such as the ECG, PPG, or continuous beat-by-beat BP signal must therefore be preprocessed. The goal with the preprocessing is to remove artefacts from the recorded signals through various methods and reliably convert the raw signals into event series, where an event corresponds to some property of one beat of the heart. Accordingly, there are as many events in the event series as there are heart beats in the raw signal. Different cardiovascular signals give rise to different event series. For the ECG, the resulting event series is called an interbeat interval (IBI) series or an RR series and each event corresponds to the duration between consecutive heart beats, typically measured in milliseconds. The term 'RR series' comes from the fact that the R-peak in the ECG waveform is used as the marker for a heart beat and each event is the time from one R-peak to the next R-peak. Similarly, for the PPG the event series is an interpulse interval time series reflecting changes in blood volume in the tissue, which varies with the action of the heart. For the continuous BP signal, it is possible to form, for example, three event series wherein each event in the respective series 
corresponds to the systolic blood pressure (SBP) for each heart beat, the diastolic blood pressure (DBP), or the mean blood pressure (MBP).

Panel a in Figure 3.1 shows a 100-second sample of ECG data from the Physionet (Goldberger et al., 2000) Fantasia database (Iyengar et al., 1996). R-peaks in the ECG have been identified and are shown in red. The resulting IBI series is presented in Panel $\mathbf{b}$, and Panel $\mathbf{c}$ shows a shorter, five-second segment of the ECG signal.

(a) ECG signal

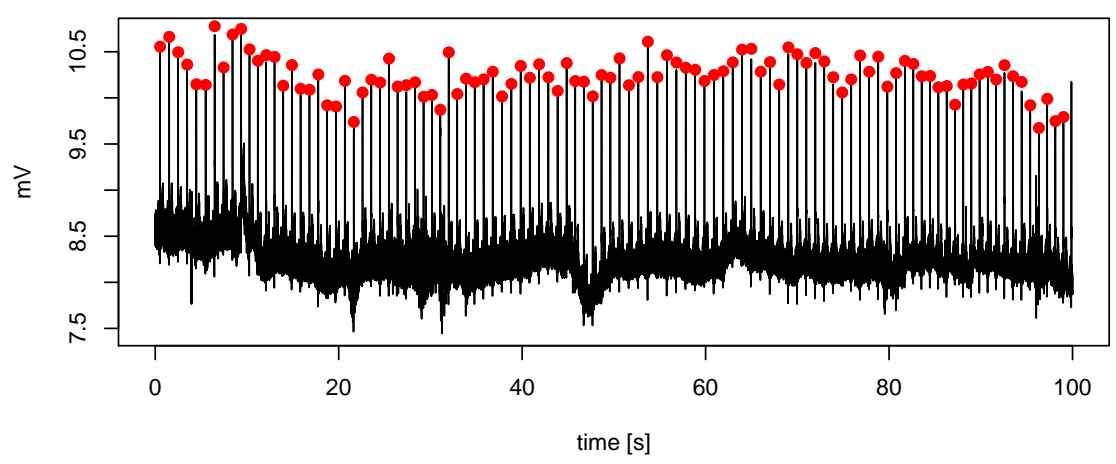

(b) Interbeat Interval Series

(c) Short ECG sample
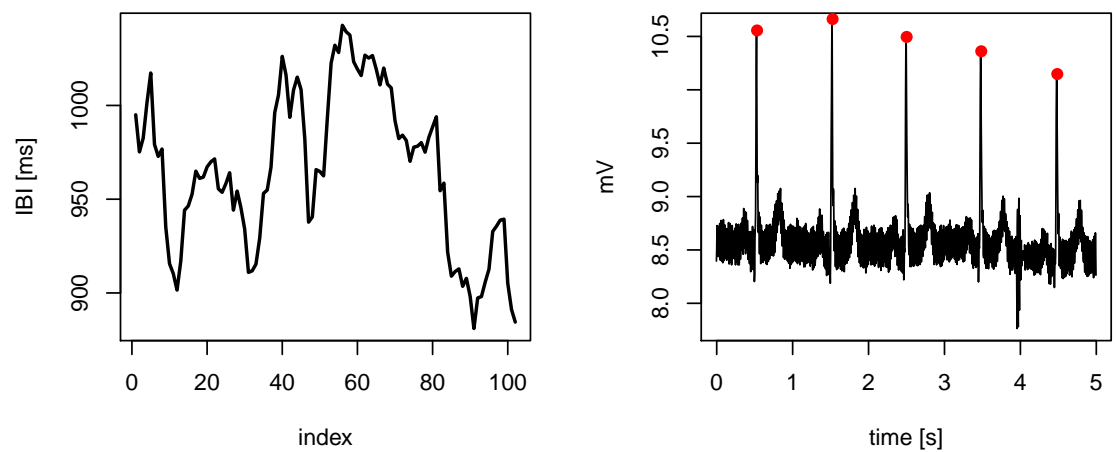

Figure 3.1: Example of the formation of an inter-beat interval (IBI) series from an ECG. The R-peaks in the ECG are shown in red. Panel a shows a 100-second ECG signal, and the corresponding IBI series is shown in panel $\mathbf{b}$. Panel $\mathbf{c}$ shows a five-second segment of the ECG signal. 
The variability of cardiovascular signals is studied by taking into consideration several distinct variability metrics calculated from the event series: HRV from the ECG (Malik et al., 1996; Berntson, 1997), blood pressure variability (BPV) from the BP signal (Parati et al., 1995), and pulse rate variability (PRV) from the PPG (Constant et al., 1999). The calculation for these variability metrics constitutes the main part of the analysis of the cardiovascular signals. The variability metrics for the individual signals can be calculated by means of several methods, such as (i) time-domain, (ii) frequency-domain, and (iii) nonlinear methods. It should be noted that, although the cardiovascular signals are of a different nature (e.g., IBIs or SDP values), many of the analysis techniques developed for HRV analysis are applicable also for BP analysis (Tarvainen, 2004). We discuss some HRV metrics next. Two examples of time-domain measures are the standard deviation of inter-beat intervals (SDNN), reflecting overall HRV, and the square root of the mean of the squares of the IBIs (RMSSD), reflecting shortterm HRV (Malik et al., 1996). The analysis in the frequency domain is based on the power spectrum of the IBI signal, derived by using the Fourier transform, an autoregressive method, or the Lomb-Scargle method (Clifford et al., 2006). In the spectral analysis, the power of the signal is considered primarily in three bands: the very low-frequency (VLF) band $(0-0.04 \mathrm{~Hz})$, the low-frequency $(\mathrm{LF})$ band $(0.04-0.15 \mathrm{~Hz})$, and the high-frequency $(\mathrm{HF})$ band $(0.15-0.40 \mathrm{~Hz})$. The LF band is typically linked to sympathetic activation and the HF band to parasympathetic activation (Malik et al., 1996), and the ratio of power in the $\mathrm{LF}$ band to power in the HF band ( $\mathrm{LF} / \mathrm{HF}$ ratio) is used to describe the degree of sympathovagal balance; see Billman (2007) for a discussion addressing issues related to the interpretation of the $\mathrm{LF} / \mathrm{HF}$ ratio. The nonlinear analysis methods involve use of metrics such as various entropies.

There are relationships among cardiovascular signals; for instance, the correlation between HRV metrics derived from fingertip PPG and from the ECG has, in general, been found to be high (Selvaraj et al., 2008; Lu et al., 2009; Lin et al., 2014), although confounding factors such as respiration should be taken into account (Lee et al., 2010). 
It ought to be noted that, though HRV and PRV are related, they are not identical (Constant et al., 1999; Lu et al., 2009; Lin et al., 2014). In addition, factors such as ambient light can affect the PPG signal, and the latter signal is less stable than the ECG during physical activity.

For a discussion of the analysis of BPV, see de Boer (1985) and Tarvainen (2004). Blood pressure reactivity can be studied also in terms of baroreflex sensitivity (BRS), which uses a combination of ECG and $\mathrm{BP}$, as illustrated in other works by, for example, Mulder et al. (1990) and Van Roon et al. (2004). The BRS metric describes how rapidly the heart rate responds to changes in blood pressure.

\section{Applications}

The most interesting phenomenon from the perspective of humancomputer interaction is how the various cardiovascular metrics reflect mental workload. It is this aspect of study to which we direct our focus in the primer.

Heart rate variability The relationship between mental workload and the ANS response, reflected in HRV, is complex. However, one can state that HRV generally is reduced during mental effort, with the degree of reduction dependent on the level of mental effort (Mulder et al., 1993). It has been shown that average heart rate is one of the most sensitive metrics for distinguishing between low and high levels of mental workload in a computerised task (Henelius et al., 2009). In addition, HRV has been used in occupational settings, a review of which can be found in the work of Togo and Takahashi (2009).

Garde et al. (2002) used HRV to investigate the difference between two computerised tasks (one using a keyboard, the other using a mouse) and concluded that no difference was evident in terms of mental workload. Differences in HRV metrics were found when the setting featured a physically demanding computer task. In a study (Hjortskov et al., 2004) investigating differences in a computerised task with different difficulty levels, the researchers found differences in the HRV LF/HF ratio between the tasks. Hjortskov and colleagues also concluded that HRV is more sensitive than BPV is to mental stress levels. In a recent study, 
Taelman et al. (2011) found several HRV metrics (average normal-tonormal interval length, SDNN, RMSSD, pNN50, LF, and HF) to be affected by the extent of mental load.

A study by Cinaz et al. (2013) investigated the use of HRV for classifying levels of workload during office work. They found that RMSSD and pNN50 decreased with the degree of workload experienced, while the $\mathrm{LF} / \mathrm{HF}$ ratio increased

In an example of particular frequency bands within the HRV spectrum, Nickel and Nachreiner (2003) investigated the $0.1 \mathrm{~Hz}$ component of HRV during the performance of a battery of computerised stress tests. They concluded that this particular frequency component was not sensitive to workload level.

The heart pre-ejection period (PEP) (Backs et al., 2000; Miyake et al., 2009) and the T-wave amplitude (TWA) (Myrtek et al., 1994; Vincent et al., 1996) too have been linked to mental stress. See the work of Lien and colleagues Lien et al. (2015) for a study comparing these indices as metrics of sympathetic nervous system activity in ambulatory settings.

Heart rate variability has been studied extensively in connection with measuring the task difficulty or mental workload experienced by pilots (Jorna, 1993; Roscoe, 1992, 1993). In addition, research by Wilson (2002) investigated various psychophysiological measurements during flight and found that HR was more sensitive than HRV to task difficulty.

Blood pressure Measurements of BP, BPV, and BRS have been applied in multiple studies related to mental workload.

For instance, in a study (Stuiver and Mulder, 2014) that considered two simulated tasks (ambulance dispatching and a driving simulator), the researchers found that HR increased during the dispatch task; BRS decreased; and BP showed an initial increase, after which it continued to rise, albeit more slowly. For the driving task, BP initially increased but then fell to near baseline levels. Both BRS and HR decreased during the task. The researchers concluded that there are task-specific differences that lead to different types of autonomic control. 
A study carried out by Madden and Savard (1995) utilised a computerised quiz to induce mental stress. It was found that BPV decreased and systolic BP rose as the degree of mental stress increased. Similar results were obtained in another study (Ring et al., 2002), in which mental stress was induced by mental arithmetic, leading to an increase in mean BP.

Finally, a study by Robbe et al. (1987) investigated BRS during mental arithmetic and memory tasks. Its conclusion was that the modulus (the gain between BPV and HRV (Mulder, 1988; Robbe et al., 1987)) decreased during task performance. Additionally, blood pressure was recorded during flight in research conducted by Veltman and Gaillard (1998), and the modulus was found to be a good index for mental effort.

Photoplethysmography In addition to the measures discussed above that reflect various aspects of cardiac variability, the autonomic activity can be studied by observing vasoconstriction. As noted above, mental stress is reflected in peripheral vasoconstriction, which is visible in the PPG signals as a decreased pulse amplitude (PA). For instance, Iani et al. (2004) investigated the peripheral arterial tone (measured by means of a pneumatic plethysmograph) during the performance of a computerised memory task. They found that subjects exhibited vasoconstriction during the more demanding memory tasks in their experiment. Similar results were found in an experiment involving simulated flight, wherein vasoconstriction was observed during difficult phases of the simulation (Iani et al., 2007).

Pulse rate variability can also be analysed in a fashion similar to HRV. Yoo and Lee (2011) examined detection of mental stress by using the PPG signal and PRV, while other researchers (Yashima et al., 2006; Kageyama et al., 2007) have explored the use of wavelet analysis for mental stress detection via the PPG signal. Arai et al. (2012) estimated mental stress by considering the $\mathrm{LF} / \mathrm{HF}$ ratio calculated from the PPG signal, and the resulting extracted mental stress metric was used to control the functioning of a mail program on a smartphone. 


\section{Conclusions}

Cardiovascular signals and the variability metrics extracted from them are used extensively for the determination of mental workload during various types of task performance. Of the signals considered in this section of the paper, the ECG and PPG signals are easy to measure, and good-quality recordings can be obtained with affordable devices such as sports watches. The recent incorporation of PPG sensors into several wrist-worn devices means that such devices could well be usable for long-term measurements. The use of remote PPG techniques, of various types, that employ ordinary webcams might be highly suitable for computer work. Blood pressure, however, is not well suited to the prolonged measurement of cardiovascular activity at present, on account of the technical requirements. In terms of usability, ECG and PPG measurements are hence currently more suitable for human-computer interaction applications than is BP.

The metrics used for analysing cardiovascular signals are well established, as illustrated, for example, by Malik et al. (1996). Furthermore, other metrics describing variations in cardiovascular signals are being studied, such as the fractal dimension of the HRV signal (Nakamura et al., 1993; Cáceres et al., 2004).

Cardiovascular metrics have been applied extensively for determining and monitoring levels of mental workload. However, it appears that these metrics have not been used thus far for the purpose of adapting user interfaces to the degree of mental workload. This is a new, relatively unexplored area.

\subsection{Electrodermal activity}

'Electrodermal activity' (EDA) is a general term used to describe changes in the electrical properties of the skin resulting from autonomic nervous system functions (Dawson et al., 2000). These fluctuations are caused by activation of sweat glands that are controlled by the sympathetic nervous system, which autonomously regulates the mobilisation of the human body for action. Furthermore, skin conductivity is not 
influenced by parasympathetic activation. Therefore, EDA can be considered to act as an indicator of both psychological and physiological arousal and, by extension, as a measure of cognitive and emotional activity (Dawson et al., 2000; Boucsein, 2012).

\section{Background}

EDA has been investigated for well over 100 years, with a number of changes having occurred in the method and the understanding of the phenomenon. Terms have changed accordingly, though 'galvanic skin response' is still commonly in use, which can be confusing; instead, one should use the modern terminology, as outlined in Boucsein et al. (2012):

"[The] first two letters refer to the method of measurement ... SP for skin potential, SR for skin resistance, SC for skin conductance, SZ for skin impedance, and SY for skin admittance. The third letter refers to level (L) or response (R)".

These terms are derived from the methods employed to detect changes in the electrical properties of the skin, which are the following: the passive measurement of electrical potential difference, or the endosomatic method, and active exosomatic measurement, wherein either alternating current (AC) or direct current (DC) is passed between two electrodes to measure the skin's conductivity, the reciprocal of its resistance. In this section, we refer to the latter method, as it is the more widely used (to our knowledge). For full details on these methods, especially how to obtain the slightly more complicated SZ and SY terms, see the work of Boucsein and colleagues.

In the literature, EDA has most often been taken as a measure of arousal (Bradley, 2000). Several studies using a picture-viewing paradigm have shown that EDA is highly correlated with self-reported emotional arousal (Lang et al., 1993). That is, arousing pictures of either positive or negative valence result in increased EDA as compared to low-arousal pictures. This index is affected by the location 
of recording, as different skin sites are innervated by different distributions of nerve bundles, not all of which are involved in emotional responses. In simple terms, emotional response affects eccrine sweat glands, which are most densely distributed on the palms and soles, nearly four times more so than on the forehead, for example. Sixteen recording sites were explored and compared in a review by van Dooren et al. (2012), which profiled site-wise responsiveness to emotional inducement (by film clips). Their review illustrates that care must be taken in the choice of the signal feature to estimate responsiveness. The authors found also that responses did not show full lateral symmetry, so care must be taken in the decision on which side of the body to record. Picard's Multiple Arousal Theory Picard et al. (2015) suggests an explanation: that different brain areas map to different areas of the body, both contralaterally and ipsilaterally.

EDA is a commonly used physiological measure when one is studying HCI experiences (see 'Applications', below). The arousal models used in HCI studies are often uni-dimensional and bipolar, and, hence,

they can be combined with a dimension of positive-negative valence to give a circumplex model of emotions, as highlighted in Figure 2.1. However, richer models have been proposed, such as the three-system model of arousal (Backs and Boucsein, 2000). Indeed, Backs and Boucsein (p. 6) argued that this might be more appropriate for investigating the specific sensitivity of physiological effects in HCI. In brief, this model posits three systems: 'affect arousal', 'effort', and 'preparatory activation', of which only affect is indexed by EDA. The areas of the CNS that correspond to these systems are Amygdala, Hippocampus, and Basal Ganglia, respectively. The authors also provided a review demonstrating the sensitivity of EDA in technology interaction studies (p. 16).

\section{Methods}

EDA instrumentation EDA is a well-established recording method, and numerous devices exist for performing laboratory-grade measurements. These devices usually comprise wired electrodes and often a bulky amplifier, thereby restricting use to controlled environments. 
Furthermore, electrodes placed on the hand are often very sensitive to motion, thereby requiring the hand to stay quite still.

With the recent increase in the quality and popularity of wearable biosensors, several portable EDA devices have become available. Portability is appealing for both psychological research and clinical use. In psychology, wearable sensors allow experiments to take place in more ecologically valid settings (Betella et al., 2014), while in health care wearable sensors enable continuous physiological monitoring at a relatively low cost (Pantelopoulos and Bourbakis, 2010).

Non-intrusively measuring EDA in a continuous long-term manner is desirable for many, quite different fields of research and diagnostics. Popular options in this regard are wearable EDA sensors, such as the ring-mounted Moodmetric (Vigofere Ltd., Helsinki, Finland); the wristworn E4 (Empatica Inc., Boston, MA, USA); or the edaMove (movisens GmbH, Karlsruhe, Germany), which combines a wrist-worn amplifier with wired electrodes. A recent study addresses the comparability of such a wearable sensor to a laboratory-grade device (Torniainen et al., 2015).

Recording EDA measurement registers the inverse of the electrical resistance 'ohm' between two points on the skin - i.e., the conductivity of the skin in that location, 'mho'. The recorded EDA signal has two components. The slowly varying tonic component of the EDA signal represents the current skin conductance level (SCL) and can be influenced by external or internal factors such as dryness of the skin and psychological state. Superimposed on the slow tonic component is a rapidly changing phasic component, skin conductance response (SCR); see, for example, Figure 3.2. The spike-like SCR corresponds to sympathetic arousal, resulting from an orienting response to either specific environmental stimuli, such as a novel, unexpected, significant, or aversive stimulus, or non-specific activation, such as deep breaths and body movements (Boucsein, 2012; Dawson et al., 2000).

Typically, EDA is recorded non-invasively from the surface of the palms and fingers. Following Boucsein et al. (2012), we recommend recording from the fingers to the extent that this is possible. Fingers 
provide good signal characteristics, such as amplitude, and responsiveness of the signal to emotional relevance is well-established. When recording is conducted in situations that demand grasping actions, which could disturb the sensors, the soles of the feet, or the forehead, may be used also (van Dooren et al., 2012).

Preprocessing In a typical EDA analysis, the acquired signal is preprocessed and then decomposed into tonic and phasic components i.e., SCL and SCRs. The preprocessing is relatively simple: data are down-sampled or low-pass filtered, typically to $<10 \mathrm{~Hz}$. Electrode displacement tends to generate artefacts, represented by signal discontinuities. These can be detected by a maximum signal-change threshold criterion and handled epoch-wise by rejection or temporal interpolation. For group analysis, the signal should then be standardised or centred.

Signal decomposition can be performed via a number of methods, depending on whether stimulus events also have been recorded. If event times are known, latency-based detection of SCRs can be performed, per Boucsein et al. (2012). Boucsein and colleagues also define the SCL as the signal in the absence of SCRs; therefore, after SCR detection, SCL can be estimated by subtraction. However, data-driven methods should be preferred, to minimise errors, because SCRs do not follow uniformly from events, and events can occur in rapid succession, causing SCR overlap. A classic example is peak-and-trough detection, which is achieved by finding zero-derivative points (where the signal is flat). One can identify SCR features from the trough-to-peak amplitude and latency. This system tends to be inaccurate for stimulus events that overlap - i.e., that have a shorter inter-stimulus interval than the recovery time of the phasic peak - because the amplitude of SCRs begins to sum. See Figure 3.2 for more details.

Alexander et al. (2005) proposed a method that handles this issue, based on the deconvolution of the signal to estimate the driver function from sudomotor nerve activity and the corresponding impulse response function, the latter describing the temporal profile of each impulse of the phasic driver response and used as the deconvolution kernel in the decomposition process. 

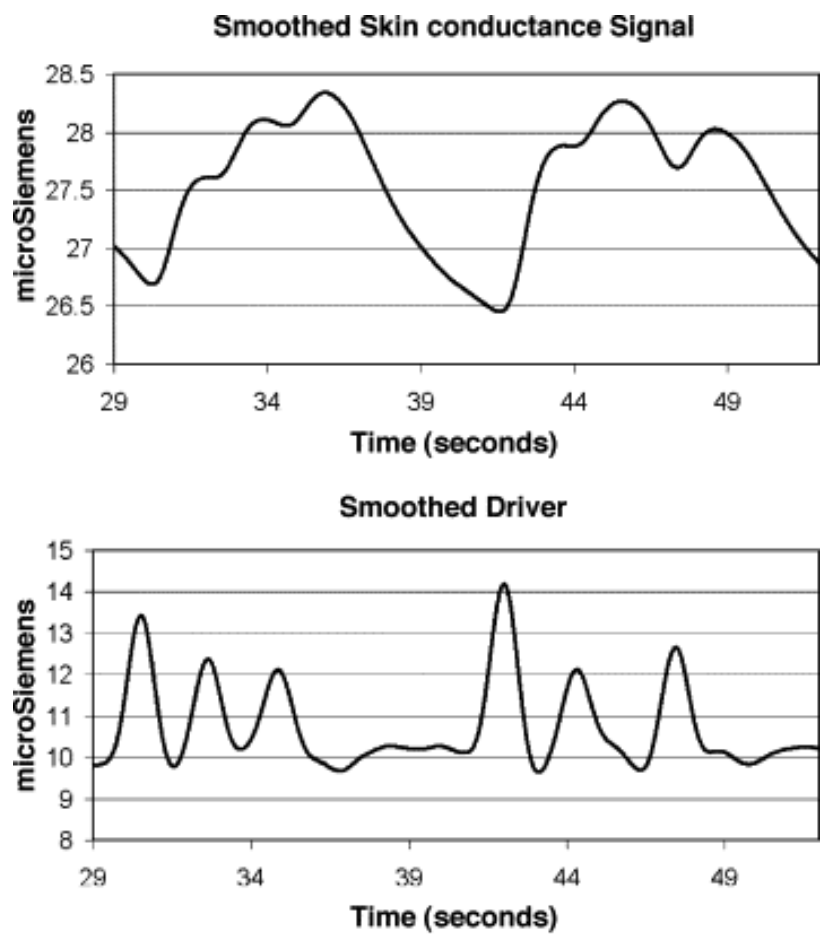

Estimated SCRs

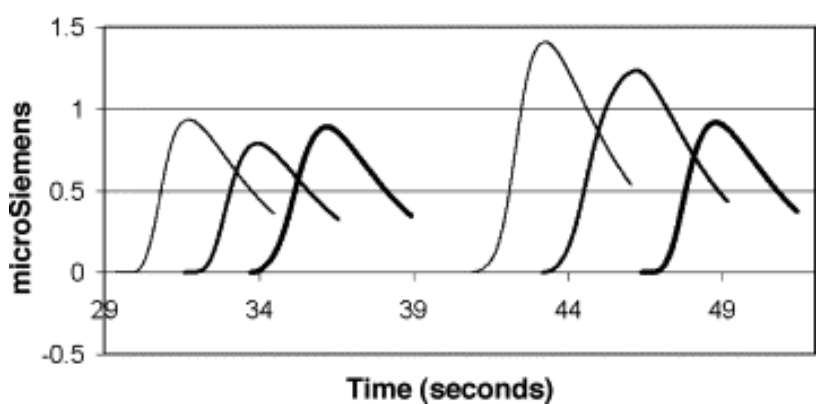

Figure 3.2: Illustration of SCR overlap, reproduced from Alexander et al. (2005), with permission. They explain: "The upper graph shows the smoothed skin conductance signal, with two groups of three overlapping SCRs. The middle graph shows the commuted driver signal, which because of its shorter time-constant has six clearly separate peaks. These separate peaks are used to estimate the individual SCRs shown in the bottom graph." 
This method is based on standard deconvolution, which does not account for variations in the SCR shape and can result in a negative driver function when the SCR has a peaked shape. These problems were addressed by Benedek and Kaernbach (2010a,b), who introduced two separate solutions: non-negative deconvolution (NND) and continuous decomposition analysis (CDA $)^{4}$. Using NND ensures that any negative component of the driver is transformed to a positive 'remainder', interpreted as the additional phasic component caused by pore opening. The output of this analysis is depicted in Figure 3.3. The NND approach was inspired by the poral valve model of EDA, which suggests that peaked SCRs result from additional sweat diffusion caused by pore opening, as illustrated in Figure 1 from Benedek and Kaernbach (2010b). They state that

"[i]f sweat ducts are filled to their limits, intraductal pressure will cause a hydraulic-driven diffusion of sweat to the corneum, resulting in a flat SCR. If intraductal pressure exceeds tissue pressure, the distal part of the duct and the pore will eventually open, which results in a peaked SCR".

CDA takes a different approach, which "abandons the concept of single, discrete responses in favour of a continuous measure of phasic activity" (Benedek and Kaernbach, 2010a). The latter is, of course, more plausible in a messy biological system. The CDA estimate of the phasic driver can take on negative values, in which case the interpretation is simply that negative values signify quality issues, either in extraction algorithm parameters or in the original data.

For Benedek and Kaernbach (2010a), estimation is a multi-step optimisation process using gradient descent to minimise a compound error consisting of a weighted sum of the negativity and indistinctness of the phasic driver. Indistinctness describes the sharpness of impulses, and negativity represents the number of negative values in the phasic driver.

\footnotetext{
${ }^{4} \mathrm{NND}$ and CDA are implemented as the Ledalab toolbox for Matlab.
} 




Figure 3.3: Screenshot from Ledalab. Top panel: 20 seconds of EDA are shown from a recording of a continuous-performance task, with inhibit (labelled 'cor-inb') and respond (labelled 'cor-rsp') targets shown every $\sim 2$ seconds. Response targets (with the subject's responses labelled 'RESP') are less frequent in the task so generate a greater EDA response. The grey area indicates SCL, and the coloured area shows SCRs diminishing over time. Bottom panel: Fitting by NND in Ledalab produces an estimate of the SCRs ('Driver', blue) and pore opening components ('Overshoot', pink).

Analysis For group phasic analyses, the impulse response function generally should be estimated separately for each participant. The phasic component is then analysed around selected events (if the phasic component was derived by data-driven methods as recommended, without reference to the events, there is the added benefit that a relationship discovered between phasic features and events cannot be an artefact of the feature extraction method). One can do this either by averaging the phasic driver or by calculating a set of phasic features and then performing the analysis in feature space (as in, for example, Khalfa et al. 
(2002)). Commonly used phasic features include the number of significant phasic peaks, the sum of amplitudes of those peaks, the time integral of the phasic response, and the maximum value of phasic activity.

\section{Applications}

EDA has seen application in a host of areas, from research to clinical practice and consumer devices. The number of form factors used in such devices remains relatively limited (they are usually situated on the wrist and fingers), but, as van Dooren et al. (2012) have shown, there are many options for recording sites. Therefore, in line with the application, the reader could conceive of implementing a device in a hat or eyeglasses (to measure forehead EDA), in socks or shoes (to measure foot sole EDA), or in a wrist-worn strap or other clothing items.

There is an extensive body of literature on EDA applications; here, we cite only a few examples.

In the area of HCI, EDA is a popular input in helping to classify arousal (usually referring to 'affect arousal' (Backs and Boucsein, 2000)). For example, Fantato et al. (2013) reported on a naïve Bayes classifier, which was trained to recognise states of affect arousal from a number of EDA features, on the basis of validated labelling of arousal levels during work-like tasks. Cross-validation testing of these tasks achieved an accuracy level above $90 \%$. The system was tested also by recording of subjects in a computer-game-like learning environment, where the classifier achieved an accuracy of $69 \%$ for predicting the selfreported emotional arousal of the game. The sensor was the VarioportARM device (Becker Meditec, Karlsruhe, Germany).

Studies have shown more specific effects also. Heiden et al. (2005), studying work done with a computer mouse, found highly significant differences in EDA between conditions that differed in the level of task difficulty. Setz et al. (2010a) compared several classifiers in discriminating between work-like tasks with a baseline cognitive load only and tasks with added stress (considered to be a form of negative affect arousal). The input consisted of 16 EDA features, and the researchers' best-performing classifier (Linear Discriminant Analysis, LDA) achieved an accuracy of $83 \%$. Their device was an early form 
of wearable arm-mounted sensor, lab-built and described in the paper referenced above.

Offering a final example, we focus on an application that is not usually connected with the workplace. Biofeedback is an increasingly popular application for performance enhancement, and it can be found in such varied contexts as clinical, occupational, and sports scenarios. In clinical biofeedback, the user is trained to respond to a given feature of the real-time signal from a physiological sensor; in this manner, the user can learn to recognise and control the subjective state that corresponds to the feature. With EDA, the feature that needs to be classified might be, for example, the number of significant phasic peaks. In an application, users could learn to recognise the subjective feeling of having more or fewer phasic peaks, then attempt to control their physiological state accordingly.

One recreational use of biofeedback involves an affect-based music player, in which concurrently measured biosignals are used to classify the listener's emotional response as the music is playing. The efficacy of such a system for inducing target moods has been demonstrated in an ecologically valid office setting, although with only a small sample size, $N=10$ (van der Zwaag et al., 2013).

O'Connell et al. (2008) demonstrated the 'Self-Alert Training' (SAT) system for EDA biofeedback, to modulate attention via arousal level. This software was validated with a group of 23 neurologically healthy participants, each of whom received brief (30-40-minute) biofeedback training sandwiched between two sustained attention to response task (SART) tests. Half of the participants were given placebo training. Analysis indicated that the SAT group

- significantly reduced their number of commission errors (a measure of response inhibition), while the placebo control group did not;

- maintained consistent response time variability (RTV - an inverse measure of sustained attention) after training, whereas the placebo group shows a significant increase in RTV; and 
- increased in arousal (SCR amplitude in response to cues) after training, while the placebo group's corresponding figures significantly decreased.

The last of these findings indicates that the short training period was enough to enable participants to counter whatever effects of fatigue and cue exposure had caused the reduction of arousal in the placebo group. This is important for the domain of safety-critical operator work in an HCI setting, where the effect of brief periods of activity to boost vigilance and alertness can be considered a valuable option for reducing human error. Such systems can now be implemented at low cost, as sensor devices are becoming robust, lightweight, and wearable, and interfaces are available for mobile platforms such as smartphones.

\section{Conclusion}

Electrodermal activity is a reliable, interpretable, and simple-to-use measure that has seen many applications in various domains. Therefore, it is an excellent choice for an introduction to the psychophysiological method and a highly suitable tool for making inferences about sympathetic nervous system activity. In addition, EDA aids in providing valuable context for other physiological signals in multimodal applications.

\subsection{Respiration}

The respiration signal is of scientific relevance because it is controlled by the autonomic nervous system and the central nervous system. This means that respiration can be consciously controlled by the subject (unlike, for instance, heart rate). Different parts of the brain are responsible for autonomic and behavioural breathing: voluntary breathing is controlled by the cerebral cortex, while autonomous breathing is controlled by the brain stem. There are, however, projections from the cortex to the brain stem that allow the higher centres to influence metabolic breathing patterns. The autonomic respiratory pattern is therefore a complex interaction among the brain stem, the limbic system, and the cortex (Homma and Masaoka, 2008). 
While respiration is an interesting signal from a theoretical perspective, there are some practical issues that limit its use in an HCI setting. Traditional respiratory measurement techniques, such as spirometers, which force the user to breathe through a tube, are often overly intrusive and cumbersome, while non-invasive methods can be too imprecise. The fact that respiration can be consciously controlled can act as a confounding factor. Also, respiration is highly susceptible to artefacts produced by speech and movement (Wientjes, 1992).

\section{Background}

Although respiration (respiration rate) is an important topic in health science, in physiology studies in a games or learning context it is less well researched than are many other psychophysiological signals, suffering from neglect even in studies of the cardiovascular system, with which it interacts intimately (Nesterov et al., 2005). This is partly an interpretative issue, as respiratory control is both voluntary and involuntary (Harver and Lorig, 2000), but equally it is a function of the uses served by respiration - the heart and brain together account for less than $3 \%$ of body weight but more than $30 \%$ of oxygen usage. Accordingly, states of low mental workload and high metabolic rate may be reflected in the respiration rate similarly to states of high mental workload and low metabolic rate. However, it has been shown that increased respiration rate results when subjects are sitting and attentively wakeful (e.g., listening to a story), as opposed to sitting without paying attention or with closed eyes. Thus, in similar metabolic situations, increased respiration indicates an increase in attention (Harver and Lorig, 2000). In consequence, given an attentionally demanding situation with static metabolic demand, respiration should increase in line with the subject's engagement. In other words, the more attention paid (barring metabolic variation), the higher the respiration rate.

In its effects, respiration is closely interwoven with most other physiological signals of interest, because it provides the oxygen that underlies localised mental activity, the energising of the musculature, and heart rate response (Harver and Lorig, 2000). Respiration affects heart rate variability through a process called 'respiratory sinus arrhythmia' 
(RSA) whereby the inter-beat interval of the heart is shortened during inspiration and prolonged during expiration (Yasuma and ichiro Hayano, 2004). It may therefore be necessary to control for breathing in experiments that include HRV.

In addition to the respiratory rate, there are several (more specific) indices that can be derived from the respiratory pattern, such as inspiratory time, inspiratory volume, and inspiratory pause. A study by Boiten (1998) explored the effect of film scenes, reaction time, and cold pressor tasks on affect-related respiratory patterns. The study showed that there were clear effects on respiratory patterns whenever the films elicited amusement or disgust, in that amusement induced a decrease in inspiratory time and tidal volume, while disgust would elicit prolonged inspiratory pauses (breath-holding). The reaction time task induced a relatively fast, shallow, and regular breathing pattern, while the cold pressor brought on a substantial increase in respiratory volume, an increase in post-inspiratory pause duration, and a large amount of breath-to-breath variability in the pattern of breathing. Accordingly, in the design of HCI applications it can be useful to pay attention to more specific details of respiration than respiration rate alone (see Kreibig (2010) for a comprehensive list of possible respiratory pattern indices).

Frequent sighing has been shown to be associated with anxiety. Blechert et al. (2006), for instance, studied the physiological correlates of anxiety and found that sighing frequency increased $150 \%$ when the participants thought that they might receive an electric shock.

\section{Methods}

Several methods exist for measuring respiration. The flow of air in and out of the lungs can be measured directly by means of a spirometer. However, while very accurate, this apparatus forces the user to wear a nose clip and to breathe through a tube, which makes it unsuitable for most HCI purposes. Therefore, instead of measuring respiration directly, it is often more convenient to measure the movement of the chest and the abdomen, by using respiratory inductance plethysmography (RIP). In this method, an elastic band is wrapped around the abdomen and around the chest. It is also possible to use only a single 


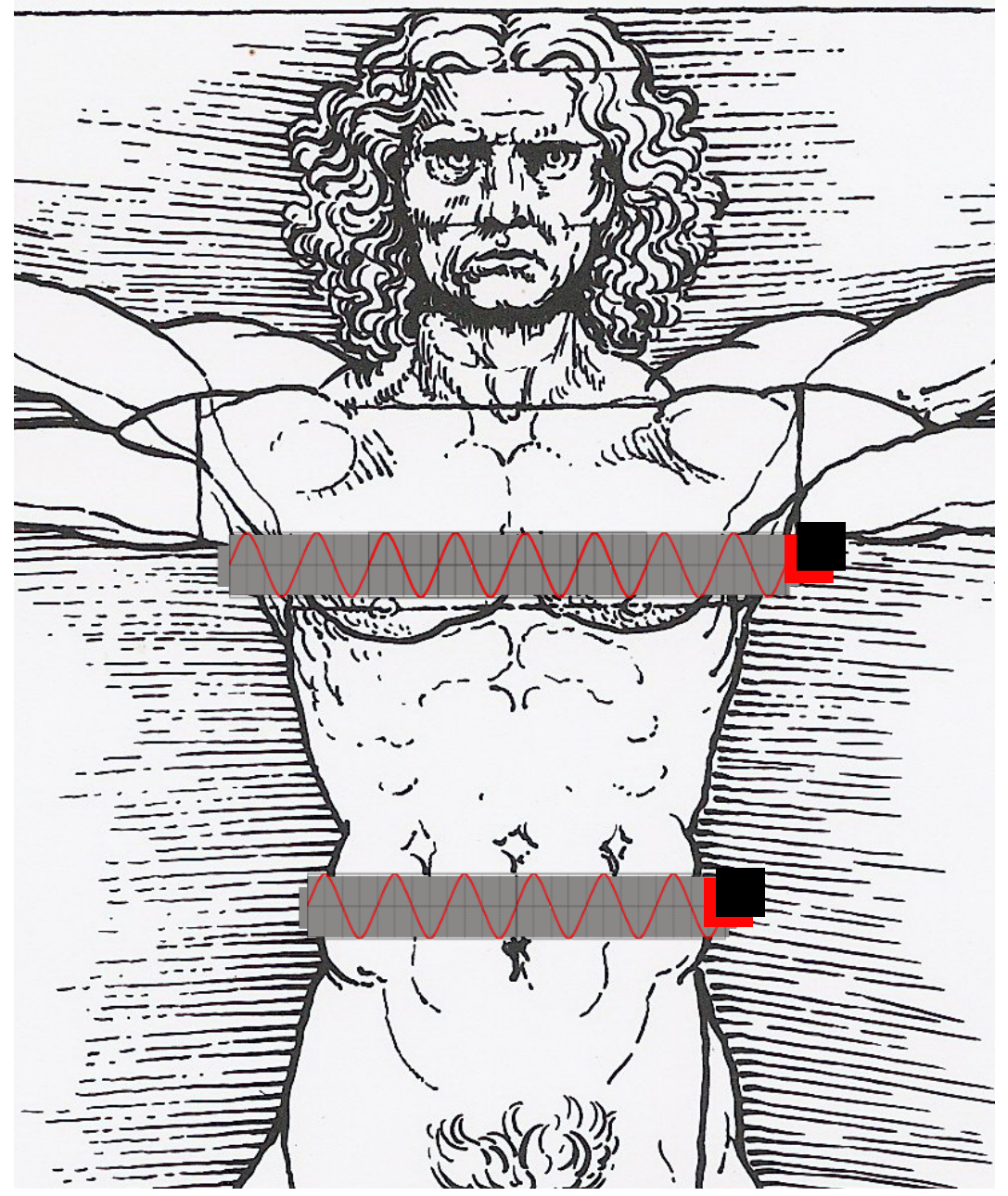

Figure 3.4: Schematic diagram of the canonical locations for respiration belt sensors, on the chest and at the abdomen. Respiration belts are typically a flexible cable transducer (pictured here as a red sinusoid) attached to an elasticated cloth belt (here, a grey rectangle), terminating in connectors to the amplifier (represented by red and black squares).

belt, around the abdomen. However, to estimate tidal volume (i.e., the total volume of air inspired and expired with each breath), it is necessary to use two belts, enabling both the abdomen and the chest to be measured (see Figure 3.4). Nonetheless, because of the physiological 
uniqueness of every individual, the measurement of tidal volume with two belts requires calibration of the system for each user, through use of a spirometer (Wientjes, 1992).

\section{Applications}

In an HCI context, respiration has been used mostly as an explicit control signal. Moraveji et al. (2011) studied whether they could influence the respiration of users during an information-processing task. Reducing breathing rate has been shown to reduce stress and anxiety, so the aim was to build a system that helps users to regulate their breathing to this end. It displayed a semi-translucent bar in the bottom half of the screen that served as guidance for the user. Users were instructed to inhale when the bar goes up and exhale as it moves down. The system was designed to illustrate a breath rate $20 \%$ below the user's baseline. It indeed reduced users' breathing rate - the authors found that users were able to decrease their breathing rate significantly while still able to continue performing information work such as research, writing, and programming.

The use of respiration in a more entertainment-oriented context was studied by Kuikkaniemi et al. (2010), who explored the use of biofeedback in computer gaming. They developed a first-person shooter game wherein the fire rate and recoil were linked to the player's respiration. In their experiment, they studied how biofeedback was perceived when the players were aware of the adaptation as compared to when they were unaware of the biofeedback. The researchers established that conscious control through respiration was more immersive and rewarding, thereby highlighting the nature of respiration as a signal that can be dealt with as either an implicit or an explicit form of interaction.

\section{Conclusions}

Respiration is a signal that is rarely used on its own. More often, is serves as an auxiliary signal either to complement information from other signals, such as heart rate in the case of respiratory sinus arrhythmia, or to serve as part of a machine learning approach that draws together information from several physiological signals. When 
used alone, respiration is usually employed as an explicit control mechanism, offering an additional input channel that can complement manual input.

\subsection{Electromyography}

Electromyography (EMG) involves the detection of myoelectric potentials by means of surface electrodes. It measures the electrical activity associated with contractions of striated muscles (Tassinary and Cacioppo, 2000). These muscle contractions may yield a direct index of the physical embodiment of various mental states, including emotions, stress, or fatigue; for example, the contraction of facial muscles underlies some emotional expressions. When one is assessing facial expression in emotion, the advantage of facial EMG measurement over observation (facial expression coding) is that it can sensitively assess hidden facial muscle activity that may not be perceptible by mere observation (Ravaja, 2004).

\section{Background}

Facial EMG has been found to be a successful method primarily in discriminating positive emotions from negative ones (Ravaja, 2004). That is, facial EMG is a psychophysiological index of hedonic valence, the dimension of emotion that ranges from negative (or unpleasant) to positive (or pleasant). For producing this index, facial EMG activity is usually recorded over three distinct facial muscle areas: the zygomaticus major (the cheek muscle area that activates during smiling), corrugator supercilii (the brow muscle area that activates during frowning), and orbicularis oculi (the periocular muscle area that activates during the so-called 'enjoyment smile' (Tassinary and Cacioppo, 2000)).

A large body of evidence shows that the processing of pleasant emotions is associated with increased activity within the zygomaticus major muscle area and that processing of unpleasant emotions evokes higher activity in the corrugator supercilii muscle area during affective imagery (Ravaja et al., 2006a) and when the subject is presented with acoustic stimuli (Bradley, 2000), radio advertisements 
(Bolls et al., 2001), emotional still and moving images (of $6 \mathrm{~s}$ duration) (Simons et al., 1999), written words (Larsen et al., 2003), textual news messages (Ravaja et al., 2006a), and news messages in video format (Ravaja et al., 2006a). There is also evidence that the zygomaticus major responds only to positive valence (pleasantness), while the corrugator supercilii responds to both negative and positive valences, in a reciprocal manner (Larsen et al., 2003). Activity in the orbicularis oculi muscle area has been correlated with pleasantness - in particular, high-arousal positive emotional states (Ravaja, 2004) - and activity in this region has also been found to differentiate smiling due to genuine pleasure from 'forced' smiling.

When one is interpreting facial EMG measurements, or facial expressions in general, it is important to know whether they provide information on the true emotional state of an individual or are social signals possibly without any connection to emotional experience. That is, there are two competing views. The emotion-expression view is that facial displays express a person's internal emotional state (e.g., Ekman (1994); see Figure 3.10, in section 3.9, below), whereas the behavioural ecology view holds that facial displays are social signals that communicate behavioural intentions or social motives (and are not 'readouts' of an underlying affective state (Fridlund, 1991)). Although the emotionexpression view is supported by a number of studies (see above), the behavioural ecology view has gained support through studies showing that positive and negative emotional facial displays are augmented in the presence of a real or an imagined audience when one is viewing videotapes, with the effect being independent of a concurrent emotional state (Fridlund, 1991). However, all in all, the evidence shows that, in social situations, facial expressions are affected by both emotional and social factors (e.g., Hess et al. (1995)).

In addition, a more straightforward interpretation of EMG signals has been posited, in which facial EMG measured from the temporalis, masseter, and medial and lateral pterygoid muscles can be used to detect bruxism (grinding of the teeth) (Lavigne et al., 2008). Among the possible causes for bruxism while one is awake are stress, anxiety, and hyperactivity. Measurement of EMG from muscles around the neck and 
shoulder region (such as the upper trapezius and deltoid muscles) can be used to monitor the fatigue of an office worker, for example (Mathiassen et al., 1995). Measurements in the area of the upper trapezius can be used for indexing shoulder-neck load, but these are sensitive to arm motions (Mathiassen et al., 1995).

\section{Methods}

The elementary functional unit of musculature is the motor unit, consisting of a motoneuron and the set of consonant muscle fibres it innervates. Muscles act more or less quickly and precisely in proportion to the innervation ratio - i.e., the number of muscle fibres per motoneuron - and this has implications for interpreting the spectral signature of muscle action potentials. For example, such potentials at a given frequency band and power level might correspond to meaningful activity if measured from the orbicularis area but may fall below the threshold required for distinguishing signal from noise if measured from the deltoid. Muscle action potentials propagate rapidly from the motoneuron endplate across muscle fibre, and a small portion of the changing electrical field of (typically multiple) muscle fibres is conducted through the intervening fluid to the skin. Therefore, what EMG measures directly is changing electrical potentials associated with grouped muscle activity, with a possibly very broad frequency range whose characteristics are related to the underlying muscle dimensions.

Facial EMG activity is typically recorded over the above-mentioned muscle areas (on the left side of the face) by means of surface $\mathrm{Ag} / \mathrm{AgCl}$ electrodes with a contact area $4 \mathrm{~mm}$ in diameter (filled with conductive electrode gel (Tassinary and Cacioppo, 2000)). Precise placement of the electrodes in this regard is important, and, for obtaining a good-quality signal, careful preparation of the skin is necessary also, to reduce any impedance between the skin surface and the gel. This involves, for example, rubbing the skin with a gauze pad and cleansing the site with either alcohol or soap and water.

The raw EMG signal is amplified, and frequencies below $30 \mathrm{~Hz}$ and above $500 \mathrm{~Hz}$ are filtered out (in some conditions, slightly different cutoff frequencies may be applicable (Blumenthal et al., 2005; Tassinary 
and Cacioppo, 2000)). In light of the Nyquist-Shannon sampling theorem, it is important that the sampling rate be at least $1000 \mathrm{~Hz}$ (twice the highest frequency featuring in the signal). In the next stage of processing, the signal is rectified (conversion to absolute values is performed) and integrated or smoothed. There are various procedures for analogue and digital processing of the facial EMG signal (see Blumenthal et al. (2005)), but one commonly applied procedure is smoothing, which involves passing the rectified EMG signal to a low-pass filter, using a digital routine. Signal amplitude is thus interpreted as muscle activation; therefore, the final metrics for EMG are simply first-order statistics of the signal, such as maximum amplitude.

Nowadays, in addition to laboratory devices, there are ambulatory psychophysiological data collection systems, such as the VarioportARM (Becker Meditec, Karlsruhe, Germany), that enable the recording of facial EMG in real-world situations (see Figure 3.5). However, the feasibility of real-world facial EMG recordings is limited by the need to attach electrodes to the face.

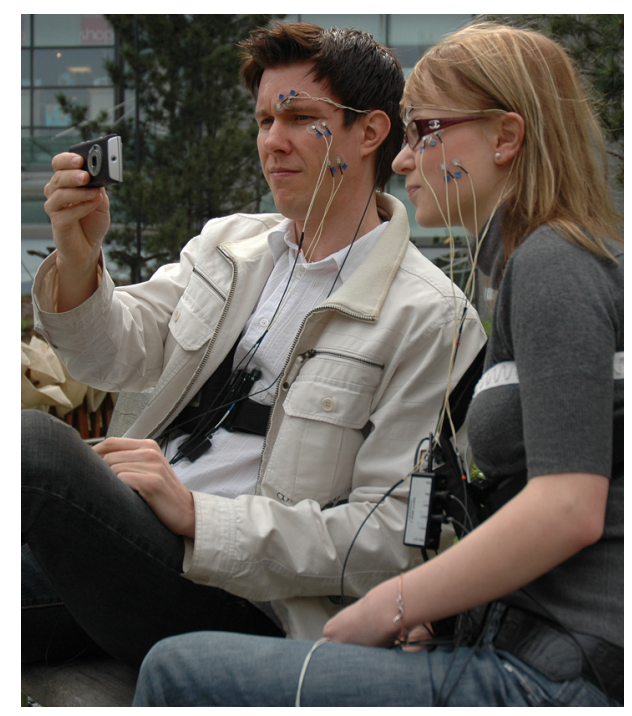

Figure 3.5: Facial EMG measurement over the brow, periocular, and cheek muscle areas via a mobile physiological data collection system in a real-world context. 


\section{Applications}

Facial EMG measurements can be applied to investigate many research questions in the area of HCI. However, as is the case for all psychophysiological measures, the interpretation of facial EMG is highly dependent on the context and research paradigm (Ravaja, 2004). It seems very likely that facial EMG provides an index of internal emotional state, especially in contexts wherein little social interaction is involved, such as viewing of emotion-eliciting material (e.g., emotional images and news items) on a computer screen. However, emotional expressions are affected also by display rules (learned rules dictating the management of emotional expressions on the basis of social circumstances, as described by Zaalberg et al. (2004)). Obviously, in some social situations (e.g., when interacting with a person of higher social status), people may even express the emotion opposite what they are feeling (e.g., smiling instead of showing anger). Clear evidence has recently emerged that display rules may influence facial expressions, and also facial EMG activity, when the subject is interacting with a virtual character (Ravaja et al., 2015). Accordingly, it seems clear that facial EMG can be used to assess emotion-bearing facial expressions but not a human's inner emotional state.

Expressing the opposite emotion from what one is actually feeling may also be done for purposes of emotional coping. For example, there is a recurrent finding that failure in a digital game (e.g., the death of the player's character in a first-person shooter game) elicits increased zygomatic and orbicularis oculi activity and decreased corrugator activity (van den Hoogen et al., 2012). That is, people tend to smile even though the game event is likely to have elicited negative rather than positive emotion. Therefore, it is important to understand that facial EMG does not index inner emotional state in connection with such a game event, even though it may be non-problematically related to experienced emotions in connection with other types of game events. Also, Ravaja et al. (2006b) also examined the influence of opponent type on facial EMG activity in playing of digital games. With both co-located and non-co-located players, they found that self-reported pleasure and zygomatic and orbicularis oculi EMG activity increased and corrugator 
EMG activity decreased in the following order: playing against a computer - playing against a stranger - playing against a friend. In this study, facial EMG activity was paralleled by self-reported emotional valence.

Recently, Salminen et al. (2013) studied the effects of computermediated cues of group emotion on facial EMG activity when the members of a non-co-located group performed knowledge-work tasks. Negative cues of group emotion (depressed or nervous/stressed) displayed in textual form on a Web page elicited lower self-reported pleasure, less perceived confidence in other group members, and higher corrugator supercilii EMG activity than did cues of positive group emotions (pleasantly excited or pleasantly relaxed). This finding was apparently due to emotional contagion. Thus, facial EMG activity appears to measure inner emotional state in the context of distributed knowledge work, at least when social communication with facial expressions is not relevant (cf. video calls). However, it was also found that planning (creative) tasks elicited lower corrugator EMG activity when compared with routine tasks. Given that routine tasks (checking the grammar of text excerpts, for instance) require effort and that attention and corrugator activity may increase with attentional effort (Cohen et al., 1992), differences in corrugator EMG activity between task types may be explained by attentional requirements. Accordingly, it should be recognised that a given psychophysiological parameter, such as corrugator EMG activity, may index different psychological processes in connection with different factors in a factorial-design experiment. This underscores the importance of understanding differences between experimental conditions in terms of what facial-EMG-related psychological processes they may evoke.

The EMG technique has been applied to other locations on the body in research on ergonomics and prosthetics. For example, EMG measurements from the deltoid have been used to study the effect of posture on performance in work at a computer (Straker et al., 1997). Wearable EMG trousers can register the activity of leg muscles during standing and walking (Tikkanen et al., 2013), and EMG can also be used as a control signal for prosthetic limbs. Additionally, it can act as 
an input signal for controlling a computer, whether in everyday applications or as a prosthetic control signal for industrial limb augmentation or rehabilitation. In everyday applications, multi-channel EMG is often used in combination with an accelerometer for gesture recognition in connection with various commands (Zhang et al., 2009). In the case of hand and arm prostheses, the EMG electrodes are usually fixed to one of the antagonist muscle pairs (Zecca et al., 2002).

\section{Conclusions}

It seems apparent that facial EMG can be a valuable measure in the area of HCI. As with all psychophysiological signals, interpretation of the data may pose challenges, though, and only under certain circumstances can facial EMG activity be used to index an individual's inner emotional state. These facts do not, however, diminish the value of facial EMG measurements, and there may be just as much value in obtaining information on emotional facial expressions determined by display rules in computer-mediated communication.

\subsection{Oscillatory electroencephalography}

Recording electrical brain activity is useful in studies of human cognition. One of the techniques available, electroencephalography (EEG), provides this measurement in an affordable and non-invasive way. In addition, when compared to other functional brain activation recording techniques, it is relatively easy to set up, mobile, and suitable for recording outside a laboratory setting. The technique measures the summated activity in local populations of oriented neurons. This synchronous activation often elicits rhythmic oscillations with distinct frequencies. The subsequent volume conduction of these oscillations can be detected at the scalp. In the strictest terms, a neural oscillation is defined as an increase - i.e., a spectral peak - in the power of the EEG signal in a specific frequency band (Lopes da Silva, 2013). Therefore, although the word 'oscillation' is used rather loosely in the context of EEG, a peak in the power spectrum should be present when one is seeking to identify an oscillation. A mere increase in power over a wider 
frequency domain does not constitute an oscillation in the frequency band of interest, however; on account of the weakness of neural currents, environmental electrical fields can cause significant interference in the signals measured, even for frequencies with detectable peaks. This section of the paper presents the possibilities that EEG affords for assessing cognitive and affective states in individuals. Here, we consider continuous EEG signals. Continuous signals encompass all EEG measurements of interest for our purposes apart from the event-related potentials (ERPs) that are discussed in section 3.6.

\section{Background}

The oscillatory analysis of an EEG signal consists of using spatial-, temporal-, and frequency-domain information for analysis of the volume conducted - i.e., electrical currents outside neurons' axons (explained in more detail in the next paragraph). These currents are measured as voltage differences between electrodes distributed over the scalp. Work with the frequency domain was the historical foundation of EEG analysis. Ever since the discovery of alpha waves, by Berger (1929), EEG has been considered a mixture of signals, with different frequencies, a perspective that has had strong implications for EEG analysis. Originally, four major types of sinusoidal EEG waves were recognised. These rhythms are presented, among other standard brain rhythms, in Table 3.1. In addition to temporal and frequency-domain features, one can analyse higher-order features such as synchrony and spatial distributions in EEG signals.

All non-invasive techniques employed for brain state evaluation require highly synchronised activity across neighbouring neuronal populations that results in signals measurable outside the scalp. The oscillations measurable on the surface of the scalp are rhythmic patterns caused by simultaneous pyramidal neuron action potentials in the area of interest. These patterns manifest as amplitude modulations via event-related synchronisation (ERS) or event-related desynchronisation (ERD) (Pfurtscheller and Lopes da Silva, 1999; Güntekin and Başar, 2014; Horschig et al., 2014). Figure 3.6 illustrates this phenomenon. In a concrete example, imagining a movement of the left hand leads to 


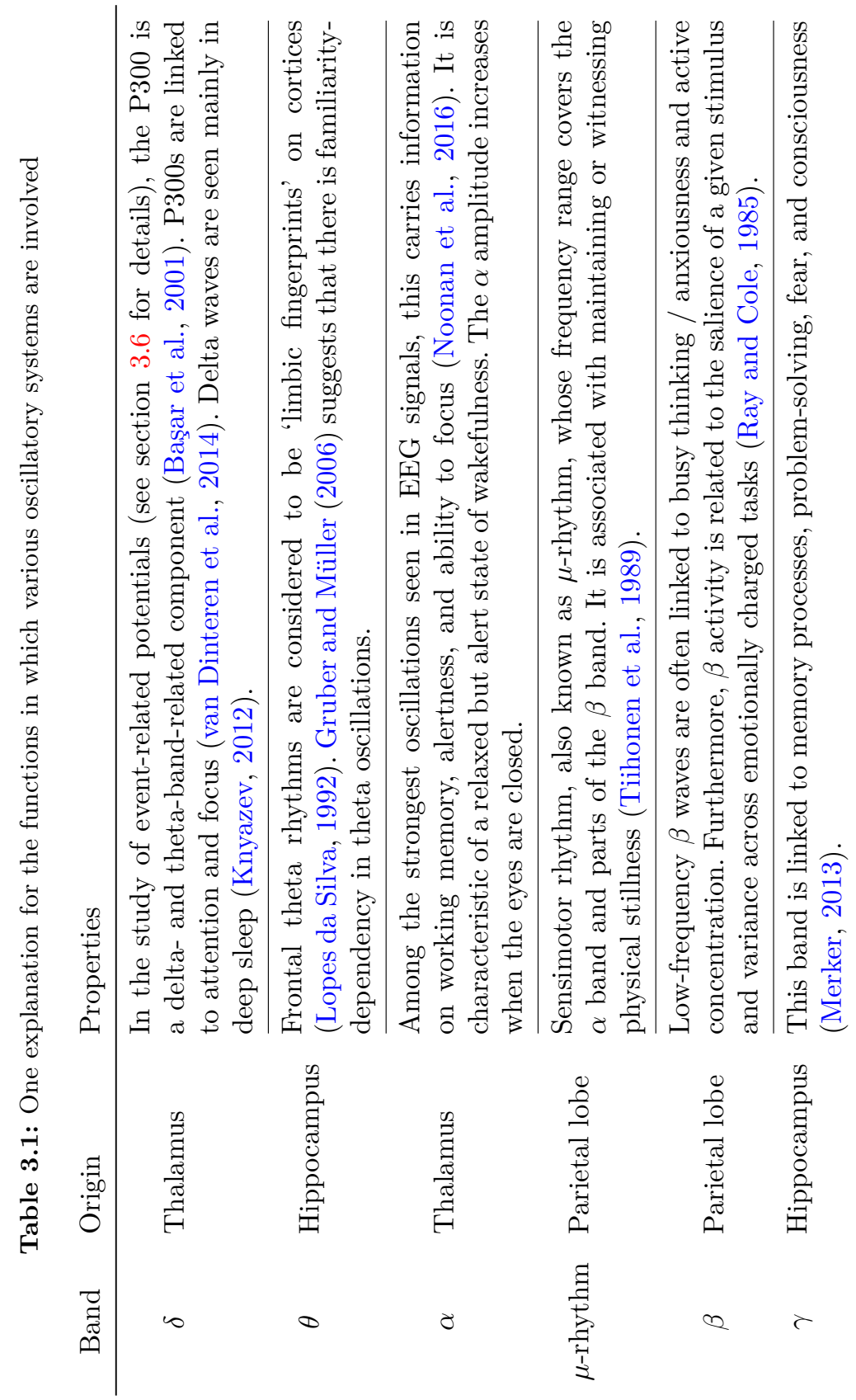




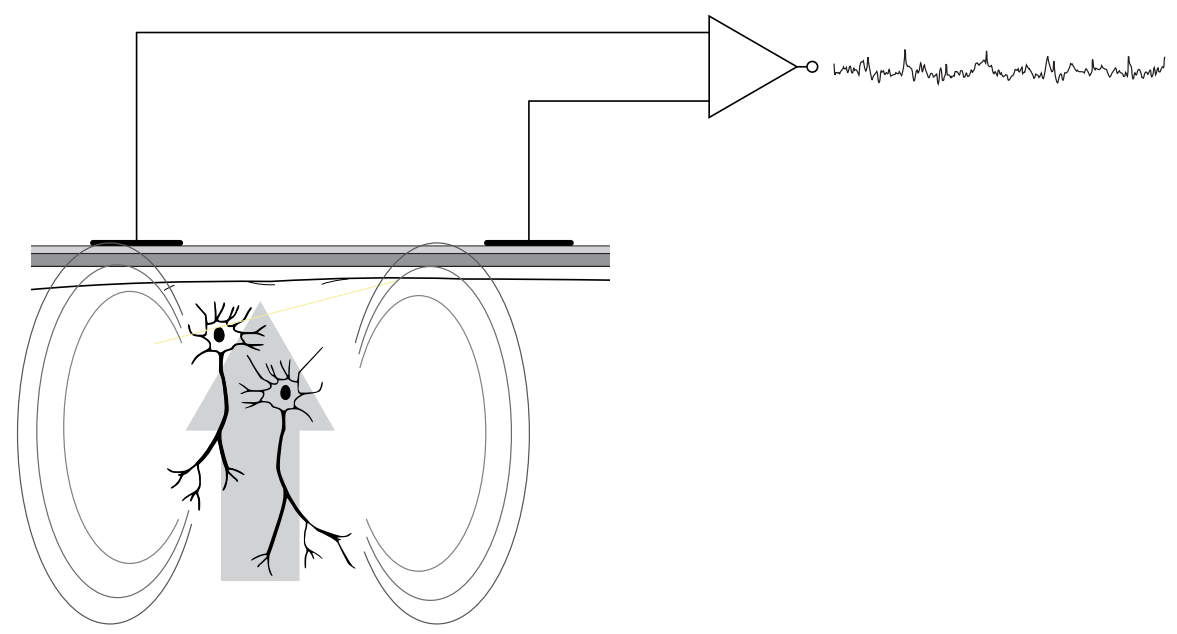

Figure 3.6: Illustration of the volume conduction of neuronal firings recorded by EEG. Physically aligned and synchronously firing neuron populations (shown superimposed on the grey arrow) induce an electrical field (represented by concentric rings). This field propagates through the layers of dura, skull, and dermis (shown as horizontal lines) and can be measured at the scalp by means of distributed electrodes. Electrical fields that are strong enough to be measured are registered as a potential difference between distinct parts of the scalp, hence the need for a reference electrode.

a contralateral ERD in the motor cortex (the right motor cortex for a movement of the left hand) in the $\mu$ and $\beta$ bands during imagining of the movement and to an ERS in the $\beta$ band (sometimes termed $\beta$ rebound) just after the imagined movement ends (Pfurtscheller and Lopes da Silva, 1999).

One example of application is brain-computer interfaces (BCIs) (Vidal, 1973) utilising information provided by various frequency bands in EEG signals. A basic design for a motor-imagery BCI - i.e., a set-up in which a computer is controlled via imagined movements - would also exploit the spatial information by extracting features only from EEG channels localised over the motor areas of the brain, typically the channels C3 (lateral parietal) for the right hand's movements, Cz (medial parietal) for foot movements, and $\mathrm{C} 4$ (opposite lateral) for movements of the left hand. The frequency bands extracted in motor-imagery design are EEG-MU (referred to as 'motoric $\mu$ ' herein) $(8-12 \mathrm{~Hz})$ and 
EEG-BETA (referred to as ' $\beta$ ' herein) $(16-24 \mathrm{~Hz}$ ). The design focuses on temporal information in event-dependent amplitude variations in these features (spatial and spectral extractions). Some generalisations pertaining to frequency-domain information that are used in experiment designs of various types are listed in Table 3.1.

Finding a correlation between EEG features and mental states is far from a simple task, and caution should be employed in generalisation from the examples presented below. They are descriptions of results from single studies and previous reviews. Researchers utilise diverse techniques to extract features from the measured signals. In addition to the features introduced above, these techniques include statistical connectivity measures, complexity measures for evaluating the predictability of EEG signal features, and chaos-theory-inspired measures that consider signals' fractal dimensions. 'Connectivity' denotes interplay of the spatially or spectrally separated signals. It is examined by assessment of basic correlation or, in more detail, by means of causality analysis (e.g., common spatial pattern (CSP) analysis), a direct transfer function (DTF), or similar techniques.

With ERD variations used as a feature, angry expressions elicit stronger beta and gamma oscillatory responses than happy and neutral expressions do when subjects view emotion-expressing faces (Güntekin and Başar, 2007; Keil et al., 2001). In addition, higher-power parietal beta activity has been found with emotionally loaded stimuli in comparison with non-emotional examples (Güntekin and Başar, 2010). The utility of simple power-based analysis techniques is limited and prone to session-to-session variations, inter-subject differences, and measurement inaccuracy. Analysing more than one EEG parameter improves the reliability of classification in respect of distinct brain functions and mental states. Results have been inconsistent between studies examining EEG band power changes elicited by emotional stimuli; hence, there is a need for in-depth analysis for all oscillatory responses, including phase-locked activity, evoked and/or induced power, time-frequency compositions, and connectivity measures. In addition, brain waves of various types are confounded by, for example, microsaccade potentials (Lopes da Silva, 2013). 
According to Güntekin and Başar (2014), future studies should examine the synchronisation of alpha oscillatory responses when subjects are presented with an emotional stimulus, to reveal the dynamics of alpha activity in full. At the same time, it must be remembered that differences in age, gender, and phenotype greatly affect EEG dynamics, and that oscillatory systems are equally important in cognitive functions. For example, alpha oscillations in the occipital areas are directly linked to cognitive performance, showing a linear relationship with attentional processes in cognitive tasks. These findings suggest that human cognition is modulated by the phase of $\alpha$ oscillations (Ergenoglu et al., 2004; de Graaf et al., 2013). Further studies support this claim, implying that even though this robust brain wave is linked to inhibitory processes, it does not represent an 'idling' rhythm of the brain but, rather, expresses 'windows of excitability' (Dugué et al., 2011).

\section{Methods}

Instrumentation EEG devices take many forms, from consumer-grade one-channel 'game controllers' to MRI-compatible medical instruments. These devices vary greatly, even at medical grade, in both their quality and properties such as number of channels, electrode type, and amplification techniques. For field experiments, EEG instrument development has produced a range of ambulatory research-grade devices. These are portable, utilise the latest innovations in electrode development, and measure a reasonable quantity of channels while remaining relatively easy to set up and use. At the time of writing, these include the LiveAmp system, from BrainProducts GmbH (Munich, Germany) ${ }^{5}$; the Quasar DSI 10/20, from Quasar, Inc. (San Diego, CA, USA) ${ }^{6}$; Enobio, by Neuroelectrics SLU (Barcelona, Spain) ${ }^{7}$; and g.MOBIlab+, from g.tec medical engineering $\mathrm{GmbH}$ (Schiedlberg, Austria) ${ }^{8}$.

\footnotetext{
${ }^{5}$ See http://pressrelease. brainproducts.com/liveamp/.

${ }^{6}$ See http://www.quasarusa.com/products_dsi.htm.

${ }^{7}$ See http://www. neuroelectrics.com/products/enobio/.

${ }^{8}$ See http://www.gtec.at/Products/Hardware-and-Accessories/g. MOBIlabSpecs-Features.
} 
Processing There are many ways to compute band power features from EEG signals (Herman et al., 2008). The canonical process flow for a basic temporal analysis of EEG data is as follows: Raw EEG $\rightarrow$ Preprocessing $\rightarrow$ Feature Extraction $\rightarrow$ (Feature Selection) $\rightarrow$ Classification (feature selection is an optional step used in subject-specified analysis).

A simple, popular, and efficient method is to band-pass filter the EEG signal from a given channel into the frequency band of interest, then square the resulting signal to compute the signal power and finally average it over time (e.g., over a time window of $1 \mathrm{~s}$ ).

Using more channels means extracting more features, which, in turn, increases the dimensionality of the data. High dimensionality leads to problems with classification and other mathematical analysis techniques. Accordingly, adding channels may even decrease performance if too few training data are available. Three main approaches can be applied in practice to exploit multiple EEG channels efficiently, all of which contribute to reducing dimensionality:

- Feature selection algorithms automatically select a subset of relevant features from among all the features extracted. These algorithms utilise machine learning techniques to perform the selection.

- Channel selection algorithms are similar to feature selection methods. They utilise mathematical routines for automatic selection of a subset of relevant channels from all available channels.

- Spatial filtering algorithms combine several channels into a single one, generally using weighted linear combinations. Features are extracted from the synthesised signal. Another kind of fixed spatial filter (Baillet et al., 2001) is represented by an inverse solution: an algorithm that enables one to estimate signals originating from sources within the brain on the basis of measurements taken at the scalp.

Alternatives for EEG feature representations can be divided into the following four categories: temporal, connectivity-, complexity-, and 
chaos-theory-related methods. Each class of methods extracts distinct attributes from EEG signals.

Temporal representations Temporal features quantify how signals vary over time. In contrast to the more basic features used for ERP, which consist simply of EEG amplitude samples over a short time window, some measures have been developed for characterising and quantifying variations in the signals measured. The corresponding features include Hjorth parameters (Hjorth, 1970) and time-domain parameters (TDPs) (Vidaurre et al., 2009). Some research results have even suggested that TDPs could be more efficient than the gold-standard band power features (Vidaurre et al., 2009).

Connectivity measures Connectivity measures indicate how signals from two channels (or signals from two anatomical locations, obtained, for instance, via spatial filtering) are correlated and synchronised, or even whether one signal influences another one. In other words, connectivity features measure how the signals of two locations in spatial or spectral space are related. This is particularly useful for BCIs and mental state assessment, since it is known that, in the brain, there are longdistance communications between distant areas (Varela et al., 2001) and that the individual frequency bands are interconnected (Palva et al., 2005). Therefore, connectivity features are put to increasing use in neuroscience and seem to be a very valuable complement to what are classed as more 'traditional' features. Among connectivity features are coherence, phase-locking values, and the directed transfer function (DFT) (Varela et al., 2001; Caramia et al., 2014; Krusienski et al., 2012).

Complexity measures Complexity measures are used for ascertaining the complexity in EEG signals. The class of complexity measures quantify regularity or predictability of a signal. This has been shown to provide information about the cognitive state of a subject and, additionally, to provide information complementary to classical features such as band power features. Some of the features in this category 
are approximate entropy, a measurement adapted from anaesthesiology (Klockars et al., 2006); predictive complexity (Brodu et al., 2012); and waveform length (Lotte, 2012).

Chaos-theory-inspired measures The fourth category of features consists of chaos-related measures, used to estimate how chaotic an EEG signal is or which chaotic properties it might possess. Among the examples one could cite of corresponding features are the fractal dimension (Wang and Sourina, 2013) and multi-fractal cumulants (Brodu et al., 2012).

\section{Applications}

The complexity of human cognition does not map well to simplicity of analyses for low-spatial-accuracy signals such as EEG output, especially when the features extracted are rather simplified, as in the case of the five established frequency bands (EEG-DELTA, EEGTHETA, EEG-ALPHA, EEG-BETA, and EEG-GAMMA, referred to as $\delta, \theta, \alpha, \beta$, and $\gamma$ ). With such a small amount of information, it is obvious that direct mapping between cognitive processes and EEG oscillations is not realistic. That said, it is likely that EEG oscillations contribute to different cognitive functions, depending on their parameters (amplitude, frequency, phase, and coherence) and their spatial location within the brain. The related functions, together with corresponding references, are listed in Table 3.1 and summarised below:

- $\delta$ : Attention and functional inhibition of executive functions (Harmony, 2013)

- $\theta$ : Hippocampal communications and the functional inhibition of executive functions (Colgin, 2013)

- $\alpha$ : Pacemaker, timing and suppressing attention (Başar, 2012)

- $\beta$ : Integrating aspects of motor and cognitive processes and affection (Kilavik et al., 2013)

- $\gamma$ : Conscious perception, updating of active memory, etc. (Merker, 2013) 
Besides the functional linkage - i.e., the causality between distant cortex areas - the interplay between frequency bands has received renewed attention. For instance, one hypothesis suggests that the capacity of working memory is represented by the ratio of brain activity involved at the theta and gamma frequencies (Lisman and Idiart, 1995). The nesting behaviour in different EEG oscillation bands itself was found long ago by Berger (1929). More recently, the power of alpha waves has been demonstrated to modulate gamma oscillations, while it has been noted in addition that cross-frequency phase synchrony among $\alpha, \beta$, and $\gamma$ is related to cognitive functions (Palva et al., 2005). It has been shown also that dysfunctions in these interplays are linked to disorders such as autism (Khan et al., 2013). For a review on crossfrequency couplings, see Canolty and Knight (2010) or Herrmann et al. (2015).

In general, the information on cognitive functions conveyed by EEG signals has been found significant in more recent studies. For instance, the EEG traces of attention, motivation, and vigilance can be utilised in the context of learning through analysis of $\alpha$ power balance on the cortices (Cowley and Ravaja, 2014). Besides the $\alpha$ frequency range, there is evidence of a $\delta$ power linkage on learning curves in 'complex video-game' environments (Mathewson et al., 2012).

\section{Conclusion}

These more sophisticated analysis methods render it possible to gain information supplementary to, for instance, basic band power features, and they may increase classification accuracy in certain conditions.

The sophisticated methods presented here provide information that can be complementary to classical EEG analysis and thereby improve, for example, the classification performance of various machine learning algorithms (Lotte, 2014).

The EEG signal processing methods presented here can be of great assistance in evaluating subject cognition, affection, and mental state. At this juncture, we must reiterate that classifying EEG signals is a tremendously complex task, on account of the non-stationary conditions, high dimensionality, artefacts, and the limited nature of the 
learning data. Current methods are far from perfect, but research is lively; for instance, methods are becoming more robust to noise, artefacts due to movement, session-to-session variations, etcetera. In turn, these advances are expected to lead towards more standardised analysis techniques for assessing human cognition. It is conceivable that they may even ultimately enable fully functional Brain-Computer Interfacing.

\subsection{Event-related brain potentials}

Event-related brain potentials are well-known and widely studied signatures of brain activity that are time-locked to a specific stimulus event, such as the beginning of a sound or the presentation of an image. Distinct from EEG oscillations, ERPs are direct representations of the time domain. These potentials are extracted from the raw EEG signal by averaging over tens to hundreds of EEG time periods (epochs) of fixed duration and are offset with respect to the time stamp for the event (Luck, 2014). Some ERP components are so strong that with a large enough number of channels and careful investigation of the spatial and spectral characteristics of the ERP, they can be recognised even without averaging (Delorme and Makeig, 2004). Such 'single-trial' ERPs, together with ERPs evoked by more naturalistic stimuli (for example, continuous ecologically valid sounds), constitute the main focus of this section. The broader subject of ERPs is quite vast; for further reading on the topic, see Kappenman and Luck (2011).

Some ERPs, especially those with low latencies, typically remain unaffected by cognitive workload, mental effort, vigilance, or affective processes, while others, especially those related to higher cognitive functions and with higher latencies, can be used reliably as indicators of mental state and task difficulty in HCI settings (Nittono et al., 2003).

\section{Background}

In electrophysiology, cognitive processes are traditionally studied via examination of high-latency neural responses of the ERP that are extracted from the continuous EEG by signal averaging. One can refer to 
ERPs in terms of their polarity (positive or negative) and the latency after the trigger event. Customarily in plots of ERPs, positive values are positioned below the $x$-axis, and latencies are denoted canonically, not exactly. Hence, for example, two common ERPs are designated as N1 and P3 (these are also referred to as the N100 and P300).

Early-going or 'fast' ERPs tend to be related to orienting responses, so the N1 ERP is a fast neuronal response to stimuli. The ERPs associated with cognition typically peak hundreds of milliseconds after the onset of an event and originate in connected cortical areas (Savers et al., 1974). Increases in task difficulty, a lack of cognitive resources, and higher cognitive loads result in a decrease in the amplitude and an increase in the latency of several ERP components. A typical example of longer-latency ERP components is the P3 (or P300), an ERP elicited in the process of decision-making. The P3 component is an endogenous ERP component related to cognitive processes such as attention, rather than physical properties of the stimulus that caused it in the first place. It can be divided into two sub-components, the P3a and P3b, which peak at different sites on the scalp. The delay in P3 (sub-)components is around 250-500 $\mathrm{ms}$ and depends on the task (Picton, 1992).

Figure 3.7, taken from reporting on an experiment that contrasted two groups (ADHD patients and healthy controls) across two conditions (illusory contour and no contour) (Cowley, 2013), illustrates some simple properties of ERPs. Firstly, N100 and P300 waves are clearly visible in all panels. Secondly, the P300 at posterior sites is much less condition-responsive than that at frontal sites. This is logical, given the back-to-front propagation of visual processing in the cortex. Thirdly, the conditions discriminate between the groups: when compared to the controls, ADHD patients show a significantly diminished P300 at F3 (above the left dorsolateral pre-frontal cortex).

Several ERP peaks are visible in responses to sound stimuli recorded while the subject is fully engaged in a primary task with a computer and not paying any attention to the sounds. The auditory P3 brain response is particularly valuable in this connection, since it has been linked to the allocation of attention to surprising, unexpected sounds separate from the primary task. The strength of this response is related to the extent 




P3
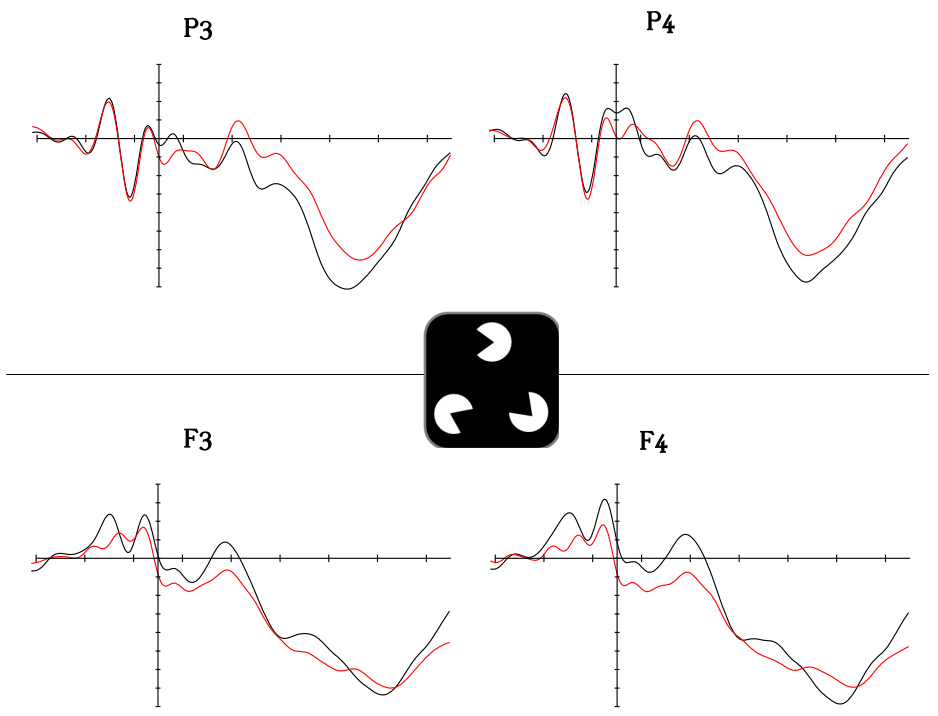

P3

P4


Figure 3.7: An example of ERPs, from four electrode sites - F3, F4, P3, and P4 (' $\mathrm{F}$ ' for frontal, ' $\mathrm{P}$ ' for parietal, '3' for left, and '4' for right) - in two conditions for a computerised response task (the condition targets are shown in black squares). The P300 is marked in the top-left panel; abscissa $(\mathrm{ms})$ and ordinate $(\mu \mathrm{V})$ scales are marked at the top right. 
of cognitive resources available at a given moment (Kramer et al., 1983). The auditory P3 is also proposed to index the momentary processing capacity of the relevant individual (Kok, 2001).

Somatosensory ERP responses have been proposed as possible indices of cognitive functions, primarily attention, during a task (Chen et al., 2014). Similarly to sounds, somatosensory stimuli can be delivered unexpectedly, without disrupting the primary task excessively.

ERPs related to a user's own actions have been proposed as markers of user cognitive state. The contingent negative variation (CNV) (Boehm et al., 2014) is a preparatory motor potential that is observed prior to movement. Its duration and strength are posited to be related to the planning of the imminent movement. Specifically, inhibition of the response is proportional to the strength of the CNV response even prior to the motor movement after decision-making (Boehm et al., 2014).

Accordingly, the 'event' for an ERP may include responses to stimuli; indeed, stimulus-event- and response-locked potentials are often analysed in pairs (O'Connell et al., 2012). In a study by O'Connell et al. (2012), the response was a subjective decision with respect to continuous (audio and visual) stimuli crossing the perception threshold. This illustrates that ERPs can be derived not only from discrete trials but also from continuous multimodal stimuli.

A further and more field-ready demonstration of ERPs from continuous natural stimuli has been provided by Poikonen et al. (2016), who extracted musical features from natural sounds and the tango nuevo, techno, and lullaby musical genres. They found that ERPs were detectable in periods of rapid feature increase, especially when such feature 'peaks' were preceded by an extended duration of low-feature values. This demonstrates the utility ERPs can have for the practitioner when combined with automated methods of extracting information from natural stimuli.

As described in section 3.5, above, cognitive processes are also associated with EEG oscillations, and the relationship between ERPs and oscillations has been investigated by, for example, Watanabe et al. (2002), who found that event-related gamma-band oscillation and the 
P3 may share several components of a common larger process related to recognising rare stimuli. Indeed, if we consider the time course of an ERP as a summation of fixed-frequency waves - e.g., the P3 is a wave with a $300 \mathrm{~ms}$ wavelength - considering ERPs to represent a brief-time-window snapshot of convolved oscillations is intuitive.

\section{Methods}

The recording for ERP signals is similar to that used for EEG applications, but it additionally requires a temporally very accurate time stamp for the events in question. This implies that the experimenter must account for transmission latencies between the EEG amplifier and the source of the stimulation event. In consequence of the very high precision required, one should also correct for time stamp 'jitter' (the slight non-periodicity inherent in any electronic signal) when events are in a stream. On the assumption that these preconditions are met, in a typical basic research setting, epochs are combined across hundreds of experiment trials and dozens of participants, to form a grand average waveform (Luck, 2014).

The process of averaging is a key factor, because the waveforms characteristic of the ERP may not be seen clearly in any of the individual trials unless they are of large amplitude. This noisiness of the individual signals can arise from source mixing and volume conduction, in addition to external contributions such as artefacts. Therefore, the ERP can be considered an idealised representation of the underlying process.

In an applied setting, one should use EEG amplifiers that are lightweight and portable to improve usefulness and affordability; even some consumer-grade devices can be used. In recent years, there has been a great increase in the commercial availability of ambulatory EEG sensors, partly due to technological advances and also on account of the popularity of the 'quantified self' movement. The sensors available range from lower-cost consumer-oriented models such as the Muse headband, from Interaxon (Toronto, Canada), with fixed electrodes, to expensive medical-grade devices such as the Embla Titanium, from 
Natus Medical Incorporated (Pleasanton, CA, USA). The Enobio (Neuroelectrics, Barcelona, Spain) amplifier is an intermediate device that is suitable for research purposes and supported in this respect (see Figure 1.1).

Consumer-oriented devices are usually accompanied by software aimed at self-quantification and cognitive enhancement; for instance, the MUSE band comes with an application that teaches meditation techniques. Devices such as this nevertheless usually employ a communication protocol that allows other software to access the device's raw data. Adding time stamps in the form of triggers, however, may prove challenging, since the solutions currently available for triggering portable EEG recorders do not typically demonstrate ERP-grade temporal accuracy. This necessitates manual verification of the trigger latency and jitter by the experimenter, a time-consuming and technically demanding task. In the field of BCI, issues of raw data access or temporal registration have usually been resolved in isolation within labs; however, this is problematic, as BCI research is largely concerned with clinical studies (Bamdad et al., 2015; Ahn et al., 2014). For these two reasons, most of the relevant reports to date have been from laboratorybased experiments. That said, the impediments to implementation outside the lab seem to be mainly an engineering problem, in terms of both data recording and design of the stimulation events.

\section{Applications}

ERP applications require that a stimulus event of sufficient information-processing novelty evoke a clear brain response. The stimulus is presented in or detected from the environment, or it may come from the behaviour of the subject during an HCI task. This implies one of the following:

- The person is going to be slightly or moderately distracted on occasion by an external stimulus (e.g., sound, a somatosensory stimulus, or light).

- The event is extracted from the task-related behaviour of the person in question (O'Connell et al., 2012). 
- The event is isolated from a continuous stimulation stream, such as a piece of music (Poikonen et al., 2016).

It should be noted with respect to external events that a secondary task related to external stimuli may annoy, distract, or fatigue the participant (Kramer and Spinks, 1991; Trejo et al., 1995; Ullsperger and von Cramon, 2001). Therefore, external events should be rare enough with respect to the primary task. In addition, the stimuli should be subtle enough not to disturb but salient enough for evoking measurable ERPs. In some cases, the strength of the stimulus, e.g. loudness of a sound, may need to be adjusted for each individual.

Studies showing the effects of cognitive load and processing capacity on the auditory P3 response have been conducted in the field (i.e. in ecologically valid settings, outside the lab) or in flight simulators (Fowler, 1994; Sirevaag et al., 1993), during simulated driving (Bayliss and Ballard, 2000), and in safety-critical monitoring (Trejo et al., 1995; Ullsperger and von Cramon, 2001). Furthermore, the P3 has been reliably extracted with freely moving subjects performing an auditory oddball task outside (De Vos et al., 2014), indicating that the relevant paradigm is nearly field-ready.

ERP paradigms can be developed to use stimuli that are elicited by the participants. For instance, the P3 difference between unexpected vs. expected stimuli can be locked to the participant's mouse clicks (Nittono et al., 2003). More recently, blink-related activity and N2 waves have been found to be predictive of user condition in a study of a simulated logistics-work environment, where the conditions involved physical effort, cognitive effort, and rest (Wascher et al., 2014).

\section{Conclusion}

Recording EEG data with portable devices and in real-world settings is non-invasive, is affordable, and exhibits a high success rate. However, the practical application of ERPs requires suitable hardware/software, which is not currently straightforward to obtain, and demands a clever stimulation protocol that is sufficient to evoke detectable responses yet not interrupt the user. Once such solutions have been identified, ERPs should constitute a valid approach for workplace HCI. Their use as a 
control input is still limited by the very low information throughput (Allison et al., 2007), but they possess a clear strength in their long history as a tool used to detect and probe cognitive states. We can conclude that using ERPs is to be recommended whenever the application clearly supports the rather strict requirements imposed by this approach.

\subsection{Pupillometry}

Pupil size has been studied largely for its value as an indicator of cognitive workload, mental effort, attention, or affective processing. Research in various fields has explored the application of pupil-based metrics in several HCI scenarios. However, the pupil is usually studied under highly controlled conditions, as it is extremely sensitive to external factors such as changes in ambient light. In fact, the pupil dilation that occurs in response to a change in light exposure is much greater than that experienced as a result of cognitive processing (Beatty and Lucero-Wagoner, 2000). Additionally, pupil behaviour is user- and session-dependent. These factors make the use of pupil size and pupil dilation indices as implicit inputs for real-world HCI settings a nontrivial challenge, yet it is an exciting one, in an area that holds great promise.

\section{Background}

Pupil size is regulated by two muscles (the sphincter pupillae and the dilator pupillae). By dilating and contracting, the pupil controls the amount of light that enters the eye; however, there are fluctuations in pupil size that are not related to the regulation of light entering the eyes. These fluctuations take place on a much smaller scale, not visible to human observers (around $0.5 \mathrm{~mm}$ ), and are associated with cognitive processes in the brain. This phenomenon has been subject to study for around 150 years now (Schiff et al., 1875). Almost a century after the first studies in this area, the field started to grow significantly (around the 1960s) and it piqued the interest of cognitive psychophysiologists 
aiming at better understanding the functions of the brain and cognition (Hess and Polt, 1964). From the large body of research that then emerged, those cognitive states achieving the greatest acceptance as able to be inferred through pupil size are mental effort and cognitive overload. In a large set of studies, researchers have shown that there is a correlation between pupil size and the level of demands imposed by a cognitive task. That is, the more demanding the cognitive task, the greater the pupil size (Beatty and Lucero-Wagoner, 2000).

More recently, technological advances in the measurement of pupil size have been accompanied by increased interest among HCI researchers in using this metric as an additional communication channel between humans and machines.

\section{Methods}

In an HCI connection, pupil size is commonly measured through videooculography (VOG), a technique using cameras (often infrared cameras) that record the eye and, by applying image processing, allow one not only to track the pupil but also to track the point of regard (the point at which the eye is looking) and other gaze-based phenomena (Duchowski, 2007), as indicated in section 3.8, on eye tracking. As noted above, changes in pupil diameter can be due either to cognitive processing, in a phenomenon also known as task-evoked pupillary responses (TEPRs), or to other types of pupillary responses, such as that to ambient light (Beatty and Lucero-Wagoner, 2000). The metrics most commonly employed to quantify TEPRs are mean pupil dilation, maximum pupil dilation (i.e., peak dilation), and latency to peak dilation. These metrics are computed about 0.5 to 5 seconds after initiation, with the time depending on the nature of the task (Beatty, 1982). For analysis of TEPRs under controlled lighting conditions it is common to divide the signal into epochs initially, in accordance with the nature of the task or the stimuli presented. For each of the epochs, baseline normalisation usually is required, in such a form as subtracting from the epoch pupil data for some period of time prior to presenting of the stimulus (e.g., 500 ms; see Beatty and Lucero-Wagoner (2000)), following which metrics can be computed. 


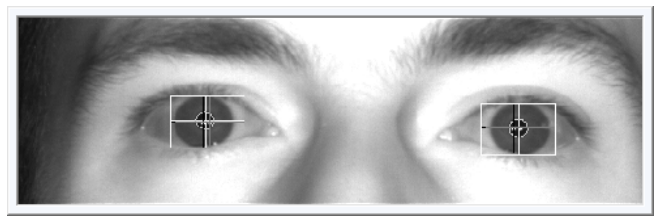

Figure 3.8: The pupil as measured through video-oculography.

A large body of research has considered the relationship between the individual elements affecting pupil dilation. For instance, Palinko and $\operatorname{Kun}(2011,2012)$ examined whether cognition-related pupil dilations can be separated from luminance-related pupil dilations in driving simulation studies. Although highly dependent on the experimental procedure, their results indicate that it is indeed possible to separate these two components of pupil dilation. More recently, Binda et al. (2014) have been studying how the interaction of luminance and cognitive load is reflected in pupil size.

\section{Applications}

To illustrate the potential for pupil size metrics in HCI, we selected a subset of state-of-the-art, non-overlapping studies in which pupil size has been used on its own rather than in combination with other psychophysiological signals.

As previously mentioned, one of the most well-established indices that can be inferred from pupil measurements is utilised for estimating mental workload. Examples of pupil size as an indicator of mental workload include the Index of Cognitive Activity (ICA), developed by Marshall (2002). In Marshall's work, pupil size is used for real-time estimation of the user's cognitive effort in the course of interaction with a virtual display. More recently, Takeuchi et al. (2011) studied whether cognitive effort as reflected in pupil size changes as the perceptual learning process progresses in visual search tasks. They found that in the early stages of the learning process, pupil size rapidly increased in line with mental effort. However, in later phases of learning, the increase was much less pronounced. Recently, Pedrotti et al. (2014) showed the 
predictive power of pupil size in relation to stress situations. They built a classifier, using neural networks, and were able to predict the stress condition being experienced by a participant by assessing pupil size. Their system achieved prediction accuracies of up to $79 \%$.

Pupil size has been used also as an indicator of affective processing. Partala and Surakka (2003) measured pupil size while participants were exposed to auditory emotional stimuli. They found that pupil dilation was significantly greater when the subjects were exposed to emotional stimuli as compared with neutral stimuli. In addition, the effect was found to be more prevalent among female subjects.

In HCI research, Oliveira et al. (2009) showed how pupil size could be useful in analysis of perceived relevance of Web search results. They studied the relevance of images and documents. Carrying out controlled laboratory experiments, they found pupil size to differ significantly when subjects were viewing relevant vs. non-relevant search results. The authors suggested that pupil size is best viewed as a delayed measure of interest, because relevant changes in the pupils were found around 400 to 500 milliseconds after the stimuli were shown. In other work, Jepma and Nieuwenhuis (2011) investigated the relationship between pupil dilation and choice strategy (exploration of new choices vs. exploitation of a fixed choice) and were able to differentiate between the two choice strategies considered: pupil dilation was significantly greater in exploration scenarios than in exploitation scenarios.

\section{Conclusion}

Pupil size has been studied in cognitive sciences for more than a century and has been slowly introduced in the HCI field in the last few decades. Pupil size can be used to infer cognitive workload and mental effort in a reliable manner, and it also can be measured relatively inexpensively and unobtrusively. However, the extensive dependence on external factors such as ambient light conditions is slowing its progress beyond controlled laboratory experiments. In a promising development, recent research aimed at discriminating task-evoked pupillary responses from external-evoked pupillary responses has shown positive results, which 
augurs a productive future for pupillometry in HCI. This may well herald application of the signal finding its way into less controlled set-ups 'in the wild'.

\subsection{Eye tracking}

As the proverbial 'windows of the soul', the eyes can be considered the only surface feature of the central nervous system. As such, they should be a rich source of information on human cognition and activity. In this brief review, we consider eye/gaze tracking as a source of information on internal cognitive and contextual state, intention, and the locus of the user's visual attention in interactive settings. Because the primary function of the eye is to sample visual information from the environment, repurposing it for direct interaction, by using gaze as an 'input method', has proved to be problematic and results in a phenomenon known as the Midas touch (Jacob, 1990): attentive information-seeking fixations are interpreted too readily as actions within the gaze-controlled interface. Eye tracking in direct-interaction applications has been discussed recently by Fairclough and Gilleade (2014) and is not discussed further here.

\section{Background}

Eye movements are highly task- and context-specific (Rothkopf et al., 2015). In a task performance context, eye movements can be divided into fixational and saccadic movements. Fixational control (consisting of fixations and smooth pursuit movements that track slowly moving targets) is aimed at stabilising the retinal image for information extraction, whereas saccadic movements rapidly move the point of gaze to fixate and acquire new features from within the visual field. Vergence (convergent, independent movement of the eyes) can provide additional information on the depth plane of visual focus in binocular viewing.

In a fairly novel development, also micro-saccades, minor directed movements of the eye within a fixation, have been shown to correlate with cognitive activity, especially visuo-spatial attention (Meyberg et al., 2015). However, as tracking these requires special equipment that 
is not usable in everyday contexts, our review will deal only with the eye movements listed above.

Eye trackers typically enable the extraction of several parameters for each type of eye movement. For fixations, typical parameters are location, duration, frequency, and drift within fixations. For saccades, the usual parameters considered are frequency, duration, amplitude, average speed, and speed profiles. In addition to eye movements, eye tracking can provide information on blinks, and typical parameters in this connection include frequency, closing of the eye(s), and eyelid closing and opening times. Among the more complex, derived parameters are dwell times (the sum of fixation times within a defined area of interest); gaze paths and patterns; the area covered; and the frequency, number of, and sequence of areas of interest visited in visual stimuli or an interface.

\section{Methods}

The two most firmly established eye tracking techniques at present are the electro-ogulogram and video-oculography. The first of these is generated from measurement of electrical activity associated with eye movements, quantified by recording from electrodes applied to the skin surface around the eye(s). The EOG approach provides high temporal resolution (up to several kilohertz) and can even be used when the eyes are closed (during sleep or at sleep onset), though it offers limited spatial resolution, has a drifting baseline, and exhibits high-frequency noise (Eggert, 2007). These properties make EOGs suitable for wearable devices tracking oculomotor parameters, but they are less useful in actual point-of-gaze (POG) tracking. Although EOG measurement set-ups vary, most often the measurements are performed with four electrodes: two horizontal, placed at the outer canthi of the eyes, with the signal summing the movement of the electrical dipoles of the two eyes, and two vertical, placed above and below one eye, which can be used also for tracking blinks.

Devices for VOG measurements are camera-based, tracking the movements of the eye via changes in visual features such as the pupil, iris, sclera, eyelid, and light source reflections on the surface of the 


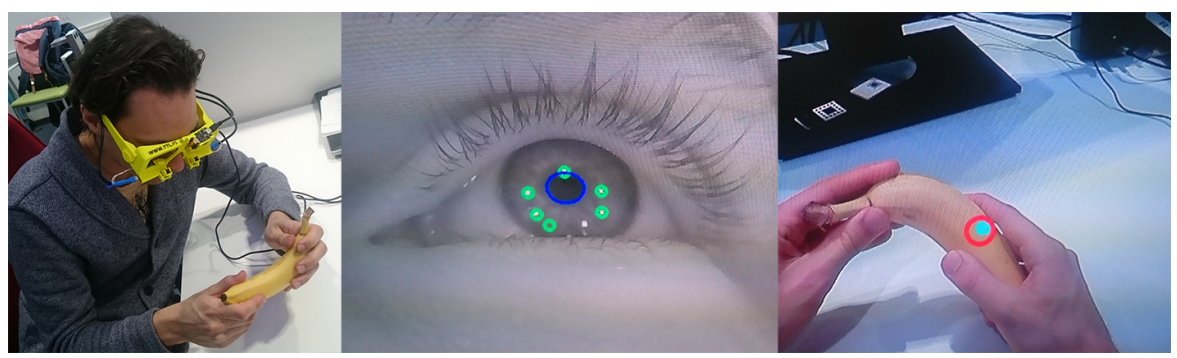

Figure 3.9: A mobile gaze tracking device in an ecologically valid application, in the left panel. The centre panel provides a visualisation of the algorithm's performance: the smaller (green) ellipses show detection of infrared light source reflections; the larger (blue) ellipse shows the subsequently detected pupil. The right panel shows the consequent detection of the point of gaze. See Lukander et al. (2013) for details.

cornea. Pupillary measures available through VOG have been linked with cognitive activity, and these are discussed in section 3.7. Videooculography devices, which can be grouped into wearable and desktop devices, vary significantly in their capabilities and requirements. Desktop devices encompass both very precise trackers, requiring the use of head and chin rests, and completely non-intrusive remote trackers that may be integrated into laptop computers and monitors. While VOG devices provide better spatial resolution than does EOG (typically $0.5-2$ degrees of visual angle), all but the most expensive tracking systems offer only limited temporal resolution $(30-60 \mathrm{~Hz})$. Hence, VOG is better suited to tracking the POG, examining gaze patterns, and utilising event-based metrics.

\section{Applications}

We will now present several example applications of eye tracking for establishing the internal state of the user on the basis of eye and gaze metrics.

Mental workload Mental workload has been studied under various task conditions. Hutton (2008) presented examples of saccadic eye 
movements being affected by working memory and attention requirements in experimental stimulus-response paradigms. Chang and Choi (2014) used gaze duration (dwell times on sentences) for measuring the spatial and temporal distribution of attention in a reading task, with 'seductive details' grabbing attention and leading to poor comprehension and recall performance.

Tokuda et al. (2011) introduced the detection of 'saccadic intrusions' - quick fixation-restoring saccades - and their frequency for estimating mental workload, while Bodala et al. (2014) utilised peak saccade velocity for gauging mental workload, claiming that higher cognitive workload necessitates faster saccades.

Flow and focus Successfully tracking flow and focus, and thereby being able to support users' task engagement, could offer a highly productive use for eye tracking. Tsai et al. (2007) reported that in a dual visual-auditory task condition, increased fixation frequency was indicative of a focus on the visual task while reduced blink frequency and horizontal vergence indicated a focus on the auditory task. Wang et al. (2014) explored gaze patterns during driving, concluding that gaze patterns contract in conditions of increased cognitive demands, focusing on a more limited area around the item of attention. Dehais et al. (2012), in the meantime, suggested an 'index of attentional focus', based on decreased saccadic activity and more concentrated fixations.

Marshall (2007) described seven binary eye-based metrics, which she used for studying cognitive state changes in three set-ups: relaxed/engaged problem-solving, focused/distracted attention in driving, and alert/fatigued state during performance of a visual search. Marshall reported that all seven eye metrics successfully discriminated among the states, with classification accuracies between $67 \%$ and $93 \%$.

Fatigue/sleepiness Fatigue and sleepiness studies have concentrated mainly on EOG, for obvious practical reasons - both blink and saccade parameters derived from EOG have been shown to be sensitive to fatigue caused by sleepiness. For blinks, Barbato et al. (1995); Khushaba et al. (2011) used blink rates and Ingre et al. (2006); Papadelis et al. 
(2007) used blink duration; while Morris and Miller (1996) showed that blink amplitude and eye closing times change significantly in consequence of fatigue. For saccades, Wright and McGown (2001); Hyoki et al. (1998) studied saccade rate and eye activity metrics, and Morris and Miller (1996); Hyoki et al. (1998); Thomas and Russo (2007) used saccadic velocity parameters for detecting fatigue and sleepiness. Another study suggested that the peak velocity of horizontal saccades could be the most powerful eye parameter for monitoring sleepiness (Hirvonen et al., 2010). Thomas and Russo (2007) presented saccadic velocity as an oculomotor neurophysiological measure significantly correlated with decreasing brain metabolism and cognitive performance, thereby demonstrating that it could be used as a surrogate marker for the for the cognitive state of alertness or sleep deprivation.

Application-specificity A large proportion of eye movement research to date has been performed in limited laboratory environments. One of the pioneers of eye tracking research, Rayner (2009), warns also that it might be hazardous to generalise eye movement metrics across task types such as reading and visual search. As eye movement metrics are highly task- and subject-specific, movements in the real world can ultimately be understood only in the context of a particular task.

\section{Conclusions}

Metrics of eye movements provide a rich, contextual source of information on human behaviour and internal cognitive states, applicable to various HCI endeavours. Recent developments in measuring and analysing ocular behaviour can inform novel tools, enabling their use in natural everyday environments. As a large proportion of the existing studies have looked at eye tracking in laboratory conditions, studying and applying gaze interaction and gaze-based user modelling in natural environments presents a substantial opportunity. However, individualto-individual variability and the task-specific nature of eye movements should be carefully considered, if one is to deliver successful applications of eye-aware user interfaces and insights into the cognitive state of users. 


\subsection{Video}

Advances in camera technologies make video an attractive possibility for measuring a variety of physiological phenomena, especially in environments such as the workplace, where contextual factors (e.g., ambient light) can be accounted for. Ever smaller and more accurate camera systems enable unobtrusive observations with sufficiently high precision for many interesting applications, and as long as there is a line of sight between the camera and the object of interest, a video signal may reveal, for example, a person's cognitive state without interrupting the work that is being done.

Here we consider digital video-based systems aimed at assessing some aspect of physiology or behaviour from a distance, in order to augment human-computer interaction. We exclude the ocular system from discussion, since it is dealt with in sections 3.7 and 3.8 .

\section{Background}

Typically, a video signal comprises measurements of the intensity of electromagnetic radiation in the spectra of visible (wavelengths of about 390 to $700 \mathrm{~nm}$ ) and infrared (wavelengths from about 700 to $1000 \mathrm{~nm}$ ) light, on a plane (for example, the image sensor in digital cameras). Changes in intensity arise mainly from a change in the original light source or the various points of reflection along the path of the ray of light from the source to the sensor. Accordingly, any movement within the measurement space (for instance, the eyebrows rising when the subject is surprised or expansion of the lungs when one is inhaling) or changes in the reflective properties of the reflection points (such as a change in skin colour due to increased blood flow) may be detected via the sensor. There is great variety in the video technologies and systems available today.

High-speed cameras A typical video camera captures 24 to 30 frames per second, depending on the encoding. While this is sufficient to make a video stream seem smooth for the human visual system, systems with 
higher frame rates have been developed too. One of the fastest methods, known as compressed ultra-fast photography (CUP), can capture non-repetitive time-evolving events at up to $10^{11}$ frames per second (Gao et al., 2014). For many physiological phenomena, a frame rate on the magnitude of 100 frames per second is adequate, and 200 frames a second may already allow, for example, the use of video-based photoplethysmography in clinical settings (Sun et al., 2012a).

Webcams The first system to feature a video camera that streamed an image in real time through a computer network came about in 1991 (Stafford-Fraser, 1995). The camera was pointed at a coffee pot in the Cambridge University Computer Lab. Since then, video cameras have become a basic feature in laptop computers and the screens of desktop computers, and they have been used mainly for video calls.

Cameras in hand-held devices While cameras forming part of traditional computers are widespread, probably the most ubiquitous camera systems today are those embedded in hand-held devices, since almost all modern mobile phones and tablet computers have one or more cameras on their faces. The main camera typically points away from the user and is intended for photography. There is often another, however, intended for video calls and points in the same direction as the screen. In addition to conveying a video image to a caller, the front-facing camera can be used to detect, for example, whether or not there is a face in front of the screen. Once a face is detected, it may reveal various attributes of the user, such as emotional engagement as assessed from facial expressions (Kang et al., 2008). Also, however, as their name suggests, hand-held devices are often held in the user's hand. It has been demonstrated that the optical sensor of a mobile phone can detect, for example, the following elements from touch: breathing rate, heart rate, blood oxygen saturation, and even atrial fibrillation or blood loss (Scully et al., 2012).

3D camera systems Whereas a single-sensor camera system typically is limited to collecting emitted visual information on a two-dimensional 
plane, adding sensors and possibly projectors to the system may enable the observation of three-dimensional structures. A system with two appropriately placed cameras (i.e., a stereo camera system) functions in the same way as human binocular vision and can produce threedimensional images. In another commonly used method, the system utilises projections of structured light patterns onto a three-dimensional surface, whereby the sensor's detection of distortions in the patterns received may reveal the precise three-dimensional co-ordinates of the surface (Ma et al., 2009). A third method, one that is quite new, uses a time-of-flight (ToF) camera system, in which distances from the source of a light pulse to a camera via each point in the visual field can be resolved from the time of flight of each light pulse on the basis of the known speed of light (Gokturk et al., 2004). Systems of this type have been used, for example, to monitor sleep (Lee et al., 2015).

\section{Methods}

With the above foundations laid, we now describe methods that have been used to extract information about human psychophysiology from a video signal. While there are diverse methods, we concentrate on three main categories here: light intensity analysis, 2D morphological analysis, and 3D morphological analysis. All of these areas are showing rapid development, and some solutions are still experimental. For practical methods, therefore, more research might be required.

Light intensity analysis is the basis for video signal analysis and enables most higher-level interpretations. Even on its own, however, simply detecting changes in the intensity of the light in a fixed area in a video image may illuminate interesting psychophysiological variables. For example, while a plethysmograph reveals changes in volume in a body, typically due to changes in the amount of blood or air contained in that part of the body, photoplethysmography is an optical technique that can be used to detect variations in the intensity of light reflected from tissue that arise from changes in blood flow (Allen, 2007a). For an optimal result, various light intensity parameters should be considered. These depend on the application. For example, pulses in line with heart 
rate seem the most apparent in the green colour channel of a colour camera feed (Sun et al., 2012b).

2D morphological analysis is the analysis of interesting areas or shapes in a 2D image, and it is based on the detection of edges between areas that differ in light intensity. For HCI purposes, the most interesting part of the body is the human face. Face detection and recognition are established research topics, and there are free tools available for these (for example, OpenCV FaceRecognizer ${ }^{9}$ ). Samal and Iyengar (1992) provide a good description of the process, from face detection all the way to the analysis of facial expressions and the classification of faces. More recently, Zhao et al. (2003) undertook an extensive review of face recognition. In a recent, thorough review, Martinez and Valstar (2016) concentrates on automatic recognition of facial expressions.

3D morphological analysis is a broad category of analysis methods that rely on different optical sensor systems producing data on 3D structures within a sensor's field of view. For example, in addition to using intensity analysis, one can collect plethysmographic data from a distance by measuring the movement of a body in three-dimensional space with an optical sensor. Even consumer-grade 3D sensors used in gaming may be utilised to measure heart and respiration rate (Bernacchia et al., 2014).

\section{Applications}

Novel video technologies and methods of signal processing give rise to interesting applications for observing psychophysiological phenomena. Here we describe two of the most interesting video-based applications for HCI: photoplethysmography and the recognition of facial expressions.

Plethysmographic data can provide basic information on psychophysiology. More thorough description is given in section 3.1. Here we consider using video cameras for PPG, an optical technique that can

\footnotetext{
${ }^{9}$ See http://docs.opencv.org/2.4/modules/contrib/doc/facerec/facerec_ tutorial.html.
} 
be utilised to detect changes in blood flow (Allen, 2007a). For example, Sun and colleagues extracted a PPG signal from a video and analysed pulse rate variability (a possible surrogate measure for HRV; see, for example, Gil et al. (2010)) from the palm of the subject's hand, using a monochrome CMOS camera running at 200 frames per second in 10-bit greyscale (Sun et al., 2012a). Tarassenko et al. (2014) measured both heart and respiration rate in a clinical set-up from a five-megapixel face video with eight bits per pixel, recorded at 12 frames per second. For broader applicability, even low-cost webcams have been demonstrated to function as photoplethysmographic sensors. In another of their studies, Sun et al. (2012b) compared a high-performance camera and a low-cost webcam in normal office lighting. They fixed a highspeed colour CMOS camera and a colour webcam in front of the face, along with a gold-standard pulse oximetry contact sensor on the index finger of the user's left hand. To ensure that they were measuring light reflected from the skin, the authors manually determined the region of interest (ROI) in each frame of the video signals. For HR detection, they used the green colour channel to analyse changes in the average intensity of the pixels within the ROI, since, especially in the webcam signal, pulsations were most apparent in this particular channel. They concluded that both imaging PPG systems can successfully measure important physiological variables (in their case, HR).

Mental state is high-level information, and identifying and conveying that information is gaining burgeoning interest in HCI research. Knowledge of the user's mental state could augment not only user interfaces but also remote collaboration, telecommuting, and video conferencing. Facial expressions are an obvious signal as to the mental state, and the recognition of facial expressions is natural (and automatic) for humans; it is an important part of our communication. Both voluntary and involuntary facial actions convey, in particular, emotional information that is otherwise difficult to express - and difficult to conceal in face-to-face interaction (Ekman, 2003). Facial expressions can be categorised as reflecting six canonical emotions in addition to a neutral expression: anger, disgust, fear, happiness, surprise, and sadness (Ekman, 1994). For an excellent review of automatic facial expression 


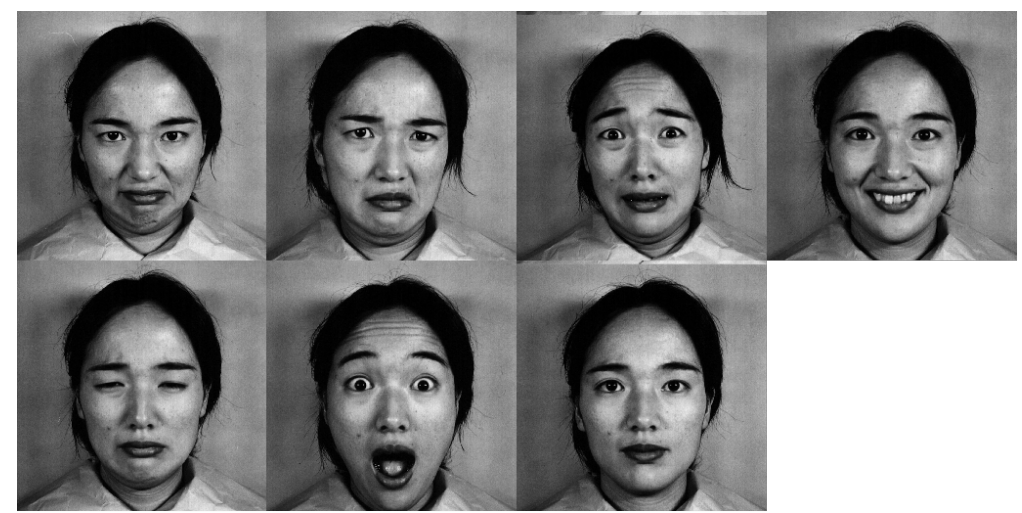

Figure 3.10: Seven actor poses for emotional expressions, from the Japanese Female Facial Expression (JAFFE) database (Lyons et al., 1998). Clockwise from top left: anger, disgust, fear, happiness, a neutral expression, surprise, and sadness. Although use of posed emotional expressions is a dated technique, such datasets provide a convenient picture of the concepts involved.

recognition, we recommend the work of Martinez and Valstar (2016). Traditionally, the process has begun with databases of portraits of actors mimicking the emotions (for example, as shown in Figure 3.10). A neutral expression is used as a reference for training the algorithm. The core challenges with such a category-based approach are that the emotions each appear relatively rarely and that some expressions may differ in meaning on the basis of context. For example, someone might smile when embarrassed, not just when happy. Another method involves looking at the basic units of muscle activity in the human face, termed 'action units', in keeping with the Facial Action Coding System (FACS; see (Ekman and Friesen, 1976)). With the FACS approach, interpretation of mental state can be done at a later stage in the analysis pipeline, with the aid of additional information on the context. A third approach is to represent the mental state on two or more dimensions of affect, such as continua for arousal (ranging from relaxed to aroused) and valence (from pleasant to unpleasant). However, neither action units nor values on the affective dimensions are always detected with the current methods, especially in real-world settings. 


\section{Conclusion}

The recent progress in video and signal processing methods renders video an interesting alternative to many traditional means of obtaining psychophysiological measurements, in areas such as plethysmography. In addition, video may enable new HCI applications, such as the remote and automatic identification of the mental state. Many of these methods are still in the early phases of development and require more research before they can achieve greater feasibility; however, there is already inexpensive hardware available, and, for many areas of study, excellent-quality free, open-source software tools and libraries exist. It is clear that the use of video in HCI is only just beginning.

\subsection{Audio - the human voice}

Human voice production originates at the larynx, where air pressure from the lungs causes vibration of the vocal folds, thereby generating a complex but patterned sound source composed of a fundamental frequency and multiple harmonics. This signal is then filtered through the vocal tract airways (oral and nasal cavities). This vocal apparatus produces a complex interactive system capable of generating a wide variety of sounds (Ghazanfar and Rendall, 2008).

Humans have evolved a nonverbal communication system in which, alongside linguistic content, speech carries rich information in the intonation, voice quality, prosody, and rhythmic variation of utterances, allowing listeners to recognise numerous distinct emotional states in the speaker. Several types of factors, from emotions to cognitive load and pathological conditions, affect the functioning of the larynx, or 'voice box', whereby the internal state of the speaker causes tightening/relaxation of the vocal folds, which modulates the acoustic and rhythmic components of speech.

Now that computing systems are starting to listen actively to people (as with Google's 'Voice', Microsoft's 'Cortana', and Apple's 'Siri'), human speech provides a promising source for online understanding of context and psychophysiological state with measurement that remains minimally intrusive. 


\section{Background}

Evaluating stress, affect, and mood on the basis of the human voice is not a new notion. Much of the literature on the effects of psychophysiological states on the acoustics of speech production has its roots in the 1970s, in work on stress and lie detectors in interrogation, military, and aerospace settings. Technologies used in these settings - namely, voice stress analysis, voice risk analysis, and layered voice analysis use the recorded features, including 'micro tremors', in a person's voice to construct a scorable 'voice gram', which is then evaluated by a specialist. While these approaches have been utilised in courtrooms and operator monitoring in demanding work tasks, their reliability has been disputed - for these methods, the most convincing results have been obtained in conditions of extreme stress, such as under threat of injury or great operational risk. The recording environments in such research has possessed heterogeneous acoustic characteristics, and the results and metrics from the relevant studies cannot be cross-evaluated reliably (Hopkins et al., 2005; Harnsberger et al., 2009).

However, even everyday user interaction situations appear to elicit strong enough emotional and stress responses to produce systematic, detectable changes in voice parameters. In the interactive setting of HCI specifically, the psychophysiological states recognised in the literature as having an effect on speech production are cognitive workload, or 'stress' (Lively et al., 1993); physical stress (Godin and Hansen, 2015), and various emotional states (El Ayadi et al., 2011).

\section{Methods}

Changes in human voice production can be measured with a microphone, in combination with the use of mathematical models to associate acoustic changes with the functioning of the larynx, or through electroglottography, in which the system uses two pairs of electrodes (one pair on either side of the subject's throat) to measure the variations over time in the degree of contact of the vocal folds during voice production (Kania et al., 2006). When microphones are employed, the approach typically involves inverse-filtering microphone recordings to 


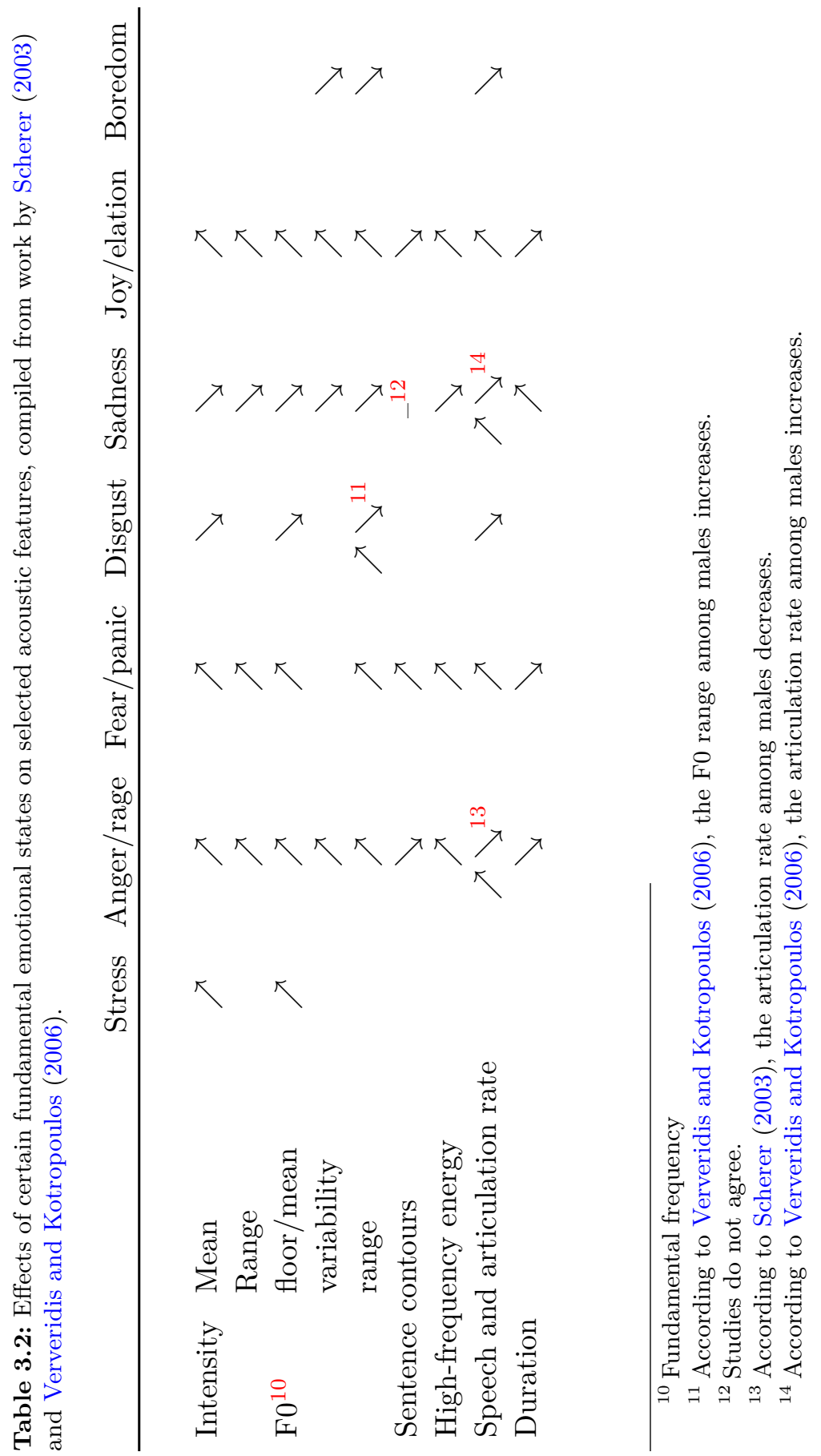


model the waveform of the glottal airflow pulses, which, in turn, reflect the movements of the vocal folds (Alku, 2011). There is a downside, however: microphone recordings vary greatly in quality and with respect to noise parameters related to microphone type, environmental factors, and distance from the speaker. Guidelines for selection of microphones suitable for human voice research are provided in a summary by Svec and Granqvist (2010).

Several derived low-level features and combinations thereof have been suggested to be correlated with variations in internal states. Table 3.2, compiled on the basis of work by Ververidis and Kotropoulos (2006) and Scherer (2003), presents the effects of fundamental emotional state on selected features. Typical features derived from speech signals include loudness, fundamental frequency, word and utterance rate (speed), jitter, zero-crossing rate, and frequency ratios. While acoustic features of speech such as pitch, timing, voice quality, and articulation have been shown to correlate highly with underlying emotional activation (low-high), there is no agreement as to how these dimensions correlate with the valence of the emotion (El Ayadi et al., 2011). Instead of direct comparisons involving individual features or combinations of them, modern approaches tend to use machine learning methods to improve detection rates (Zhou et al., 2001).

\section{Applications}

While variations between one speaker and the next require calibration and baselines, online calculation of acoustic parameters and spectral measures is relatively easy and robust. In one recent innovation, a set of open-source toolboxes for automated feature extraction and voice analysis has been developed. These include openSMILE - the Munich Versatile and Fast Open-Source Audio Feature Extractor (Eyben et al., 2010) - and AMMON (Affective and Mental Health Monitor) (Chang et al., 2011).

A considerable amount of the recent research in this field has been presented in connection with the Interspeech computational paralinguistic challenges (Schuller et al., 2015). Since 2009, these chal-

lenges have called for methods of evaluating speech for the detection 
of age, gender, affect and emotional parameters, personality, likeability, pathologies and diseases, social signals, signs of conflict, cognitive load, and physical demands. For an extensive list of examples of computational solutions for the paralinguistic detection of cognitive and emotional states, the reader is directed to the Interspeech repository available online at http://compare.openaudio.eu/.

\section{Conclusion}

For the interactive setting that is the focus of our attention, analysing the acoustics of speech production offers a non-intrusive online metric for gauging the internal state of the user. There is considerable potential on account of the pervasiveness and unobtrusive nature of the method. The classification performance of automated solutions is beginning to reach an acceptable level of sensitivity and reliability, at least upon user-specific calibration to accommodate the effects of differences in languages and dialects, individual-to-individual differences in speech production, and variations in stress and affective responses.

Another source of motivation for significant improvements in both recognising and producing natural paralinguistic cues associated with empathetic responses arises from future needs related to affective computing, robotics, and artificial conversation partners in general. These technologies necessitate naturalistic input and output down to the smallest detail in order to escape the 'uncanny valley' (Mori et al., 2012).

\subsection{Multimodal signal classification}

Having considered 10 individual types of signal source, we can now discuss their use in combination. Signals obtained from multiple sources (physiological and behavioural) can be combined and analysed collectively for determining the state of a user (or, in some cases, multiple users). Performing cognitive or affective state assessment by means of multiple signals is the focus of this section and the next. We begin by considering the theory of data fusion in general, especially when the 
fusion is performed offline (i.e., post-recording), then devote the final subsection to online data fusion.

Increasing the number of signals analysed has the potential of providing more accurate estimates; whereas data collected from a single source could lead to conflicting interpretations, using data from multiple sources in combination aids in disambiguating cognitive or affective state. In the case of the widely known two-dimensional model of affect (Russell, 1980) (a simplified version of which was depicted in Figure 2.1), a moderate increase in arousal could be caused by the subject experiencing either fear or annoyance. For the purpose of our example, we assume that this increase in arousal is unequivocally measured via EDA (discussed in section 3.2). Integrating EDA with additional measurement (such as facial recognition) can assist in discriminating which of the two emotions the user was actually experiencing; for instance, the vertical position of the eyebrows is often higher when one is experiencing fear rather than annoyance. Similarly, when facial recognition alone does not provide enough information to detect the emotion unambiguously, measuring arousal (from EDA or other signals) could aid in correctly classifying less clear-cut cases. For example, Bailenson et al. (2008) found males to be less facially expressive than females when experiencing sadness. Accordingly, including additional physiological signals in their classifier (such as EDA and ECG) increased the probability of correctly detecting sadness for males. Mandryk and Atkins (2007) presented an interesting approach, using a fuzzy classifier based on ECG, EDA, and facial EMG, to classify both arousal and valence of participants playing a popular computer game.

Novak et al. (2012) and Lisetti and Nasoz (2004) discuss general strategies that can be employed in the design and implementation of multimodal systems. Inspired by these works, we describe here a generic schema for detecting affective and cognitive state in the types of multimodal systems discussed in this part of the primer; see Figure 3.11. As this figure shows, stimuli are associated with some cognitive/affective state(s) in the first step, either through the use of validated stimuli (e.g., the International Affective Picture System) or via subjective labelling of each stimulus after perception (for instance, a questionnaire can be used to evaluate a recording of the experience). Metrics are 
computed from their respective signals. Then, a classifier is trained to associate the data obtained with specific affective states on the basis of the stimulus classification performed earlier. 'Optional' procedures, shown in dotted boxes in the figure, may be applied at this stage. Such optional procedures may increase classification accuracy. For example, one may include a user model based on population-level inferences or (non-physiological) contextual information - such as time of day. Finally, reverse inference is performed to associate a particular set of data with a single specific state or with a range of states; probabilities are used to identify those states that are more likely to be associated with the observed data.

Multimodal systems can be useful when data from a given channel are missing, a situation that can normally be expected to arise when data are recorded outside research laboratories. For example, in the research by Wagner et al. (2011a), missing data were handled by means of a naïve Bayes classifier and three modalities: voice (audio), facial recognition (video), and gestures (accelerometer).

\section{Determination of affective and cognitive state}

Several studies have considered fusing information from multiple signals in order to assess mental workload. In a recent study, Hogervorst et al. (2014) noted that the use of multiple physiological signals is expected to enhance estimation of mental workload if the chosen signals represent separate aspects of workload. They extracted features from EEG, ECG, skin conductance, respiration, pupil size, and eye blinks, using these as inputs for both support vector machine (SVM) and elastic net classifiers. They achieved a high classification accuracy in two-level workload determination.

Cognitive state can be estimated also through a combination of a single physiological signal and a non-physiological source - Mühl et al. (2014), for example, used an LDA classifier to classify mental workload, combining EEG and mood (measured via self-reporting). Fusing of multiple features from the same signal has been undertaken, for instance, by Brouwer et al. (2012), who used features derived from EEG as inputs to an SVM classifier. They concluded that models using 


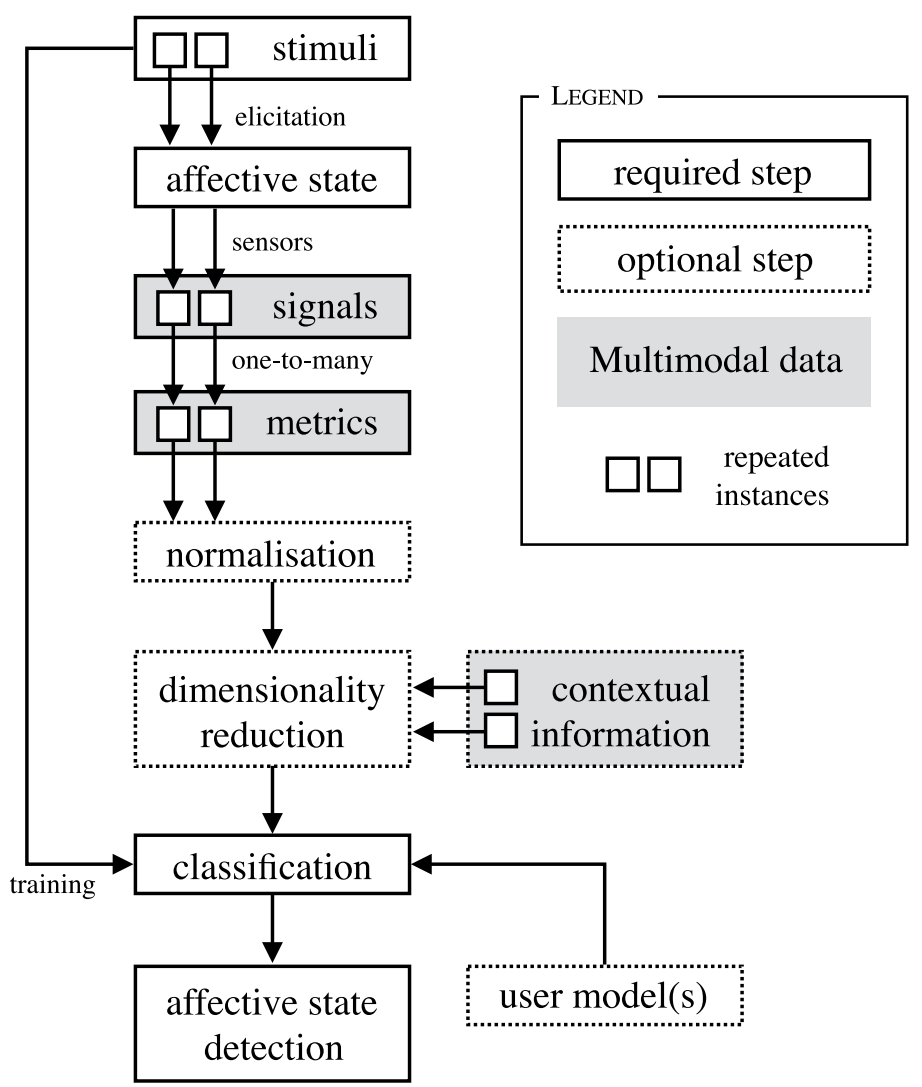

Figure 3.11: Diagrammatic generalisation of multimodal physiological systems. Grey boxes are roughly equivalent to the concept of a 'complex topography' in signal processing systems (depicted below in Figure 3.12).

both EEG and ERP features - i.e., fusion models - worked better for mental workload classification using short data segments.

Given that learning can be facilitated by an optimal level of arousal (Baldi and Bucherelli, 2005; Sage and Bennett, 1973), Cowley and Ravaja (2014) showed that the interaction of EDA and EEG features predicted learning outcomes in a game-like task wherein individual signals alone were uninformative.

A study by Wilson and Russell (2003b) applied a combination of 85 EEG frequency power features, inter-beat interval, respiration rate, 
and electro-oculogram data from air traffic controllers. These 88 features were classified via an artificial neural network (ANN), achieving very high accuracy in two-class mental workload classification. Similar performance was achieved in classification of mental workload on a three-level scale instead (Wilson and Russell, 2003c). The same set of signals were used by Wilson and Russell (2007) in an adaptive-aiding system.

\section{Applications}

Multimodal systems capable of interpreting physiological signals in realistic HCI applications have been implemented with some success, especially in the last 10 years. We will discuss the most relevant applied work here.

Healey and Picard (2005) examined drivers' stress levels (referring to distress, or negative stress; see Table 4.1) via four modalities: ECG, EMG, EDA, and respiration. Using Fisher's discriminant analysis, they reported an accuracy of $97.4 \%$. This relatively early research focused only on assessing the feasibility of correctly classifying three levels of stress. Malta et al. (2011) conducted a similar study, in which drivers' frustration was measured during actual (non-simulated) driving. Using a Bayesian network, they classified specific time segments in two classes (frustration-present vs. frustration-absent). They achieved, for the most part, $80 \%$ hit and $9 \%$ false positive rates, with the rates depending on the data fed to the classifier. Interestingly, the most accurate predictions were obtained when contextual information, such as traffic density, was provided to the classifier along with physiological information.

Pavlidis et al. (2007) utilised thermal cameras to measure multiple signals. Although only a single sensor was employed technically (the camera's thermal sensor), they effectively measured blood flow, cardiac pulse, and breathing rate. These metrics were used to compute a general level of negative stress, by means of a model of data fusion developed specifically for this application. The researchers reported good accuracy in detecting the actual stress levels ( $r=0.91$, Pearson correlation), with the exception of one outlier. They proposed two applications, desktop 
computer monitoring and sleep analysis, in both of which a stress level was computed for the current user, who was then alerted in the event that a specific threshold was exceeded. It should be noted, though, that the desktop computer test was carried out in the form of a laboratory experiment (a variant of the Stroop Test) (Pavlidis et al., 2007).

In other work, a biometric mouse and finger sensor (intended for desktop computer users) were used to measure EDA, skin temperature, heart rate, and touch intensity, in conjunction with behavioural data (such as mouse movements), for development of a recommender system that was tested in real-world working conditions over a span of four years (Kaklauskas et al., 2011). The recommender system was capable of detecting stress and anxiety. It then presented individual users with suggestions for managing their work environment, on demand. Similar research involved a multimodal system employing EEG, ECG, and EMG, which was tested by CAD engineers. The system classified four emotions (frustration, challenge, engagement, and satisfaction) in users carrying out CAD work (so that, for example, one could detect designs developed amid frustration, which might display errors in judgement). However, small sample sizes prevented statistically significant findings (Liu et al., 2013). In other applications, it was noted that less expert users might benefit from physiological integration, as in a system that utilises gaze, facial recognition, and speech recognition in order to predict user intentions. However, while welcomed by non-experts, a system of this nature (Maat and Pantic, 2007) was met with dissatisfaction by more experienced users.

Measurement of physiology from multiple sources has not been limited to single-user desktop computer usage. It has been carried out in more social settings also, such as at meetings or on public speaking occasions. For example, stress-related arousal has been measured 'in the wild' through a combination of EDA, ECG, and motion sensors (though the system was tested with only a small number of participants) (Kusserow et al., 2013). This creates the possibility of analysing one's own data during a public speaking event, so that any performance problems due to distress can be identified and, possibly, be avoided in 
the future. In addition, observer stress, the stress experienced by a person observing a meeting or group event, has been measured by means of thermal cameras, EDA, and EEG (Sharma and Gedeon, 2014). In the same study, observer stress was measured during an interview event (with the interview being designed to induce observer stress at specific moments). With genetic algorithms and support vector machines, the presence (or absence) of observer stress was detectable with an accuracy of $98 \%$. In a similar context, a more laboratory-oriented setting (which used virtual humans as 'presenters') utilised video cameras (for facial recognition), eye tracking, and audio signals to measure observer interest in a given topic (Schuller et al., 2009). That study demonstrated that a virtual agent was capable of detecting loss of the user's interest and of switching topic accordingly. Although the frequent change of topic might have had an adverse impact on user understanding, the system was capable of correctly detecting changes in user interest (with a cross-correlation value of 0.72 , using support vector regression).

\section{Challenges}

As are all the other methods mentioned, multimodal methods are affected by high noise levels at the receiving end. The 'bleeding-edge' nature of the technologies employed implies that signal quality can vary greatly across devices. Moreover, the interference typical of bodybased measurements (such as adverse effects of muscle movements on EEG) complicates processing of the signals obtained. In general, this means that 'one size fits all' measurements are infeasible, so user- and environment-based customisations are required for obtaining satisfactory signal quality, especially when physiology is being measured outside well-controlled laboratory settings (Hong and Dey, 2014).

If they are to be effective, multimodal measurements would benefit from an overarching theory of emotion. Although some approaches pointing in this direction have been put forward (Conati and Maclaren, 2009; Peter and Herbon, 2006; Gunes and Schuller, 2013; Gunes and Pantic, 2010), consensus is still lacking, as the nature of emotions is not fully understood. Moreover, a lack of interdisciplinary work is evident in 
this field: applications devised within one community are rarely shared with other communities (for example, an HCI experiment may not demonstrate strong enough correlations between the data and specific events to satisfy the demands of psychological research, while psychological research is usually too theoretically oriented for HCI applications) (Lopatovska and Arapakis, 2011).

The absence of consensus with regard to theories of emotion might also explain why multimodal methods generally manifest only modest improvements over single-sensor settings (D'Mello and Kory, 2012). Often, multimodal systems assume co-ordinated bodily responses to a single emotion, which might not occur in reality. Since multimodal measurements demand more computing power, it is important to verify that multiple theories of emotion are considered while the work is still in the development stage, so as to avoid the creation of a highly complex system that yields minimal gains over a single-sensorbased system. That said, modest gains are still welcome, provided that the increase in computation time is not unmanageable. Moreover, development of novel systems that take current theories of emotion into account could lead to new insights into the nature and physiology of emotions themselves (e.g., in the case of unexpected findings).

\section{Conclusion}

Most of the multimodal applications described here, especially in the context of HCI, have focused on detection of (negative) stress, possibly because stress is often identified as the most negative - yet measurable - factor that can arise in working environments. Applications in this area have found some success, although they rarely address use cases beyond user self-assessment. Multimodal applications that constitute attempts to detect other cognitive or affective states, such as interest, intention, or emotion, do exist but are only in the early stages of their development. For that reason, research in this connection is currently focused on improving classification accuracies rather than on presenting effective use cases. 
It appears that research into multimodal physiological systems would benefit from more interdisciplinary work focused on finding common ground between the fields of psychology and human-computer interaction, so that advanced systems, capable of detecting user states with greater accuracy, can be developed. Tools that should aid in the implementation of advanced, online data-fusion systems are discussed in the final section.

\subsection{Solutions for online data processing and fusion}

We have covered a wide range of individual biosensors and areas of application for them, and, with the preceding section, we began to discuss signal fusion. While we have thereby looked at it from a theoretical perspective, we have not yet addressed the topic of how to perform these fusion operations (feature extraction and classification) involving multiple signal sources in real time while leveraging modern stream processing methods. Therefore, the final subsection focuses on the implementation and technical aspects of real-time stream processing systems for the online extraction and fusion of indices from streaming biosignals. The calculation of user indices from one or more signals is often realised by means of machine learning techniques such as a classifier. We address the integration of these indices into various applications also, by listing currently available hardware and software solutions.

\section{An overview of stream processing systems}

Real-time data fusion requires a tool for combining multiple physiological signal streams and performing online extraction of features from raw signal data. Typical database management systems do not perform this task well, as the databases must be updated constantly for incoming data and the relevant operations tend to be slow. Also, long-term data storage is not required in most applications, since short fixed-length buffers cover the time ranges presented in Table 2.2. Systems that take these factors into consideration are generally referred to as stream processing systems (SPSs). Their origins lie in the need for 
real-time processing of high-velocity and high-volume data. Example applications can be found in the field of data-mining: fraud detection, stock market analysis, and manufacture monitoring. Although dated, the most comprehensive survey of generic stream processing systems can be found in Stephens (1997). Most existing stream processing systems are generic; i.e., they can be configured to process various kinds of data. The most prominent examples of this type of SPS are AURORA (Abadi et al., 2003) and its continuation project BOREALIS (Abadi et al., 2005). The downside of a generic SPS is the overheads required for configuring the system for a specific task. For this reason, specialised, task-specific SPSs designed to deal with particular data types have been developed. Examples of task-specific SPSs used for physiological data include brain-computer interfaces and body area networks (see Chen et al. (2011)).

Stream processing systems are made up of three basic components, often termed sources, filters, and sinks (Stephens, 1997), although the naming conventions employed in the literature vary. For the sake of clarity, these components are referred to here as sensors, processing elements, and clients, for consistency with the rest of the review; these names match the terms introduced in Table 4.1. Stream processing systems are often represented as directed graphs comprising the three above-mentioned components. Two examples of SPS architectures, with different scales, are shown in Figure 3.12.

Sensor Broadly speaking, one can define a sensor as an instrument used to measure some form of signal from a subject. In addition to the sensor element itself, this definition encompasses the interface and the protocol for transforming the incoming data into a format understood by the SPS. Sensor protocols include vendor-specific proprietary formats and APIs but also more open protocols such as the lab streaming layer ${ }^{15}$ (LSL) (Kothe, 2013). Most methods employed for recording physiology have some sensor instrument that can be implemented as part of an SPS.

\footnotetext{
${ }^{15}$ See https://github.com/sccn/labstreaminglayer.
} 

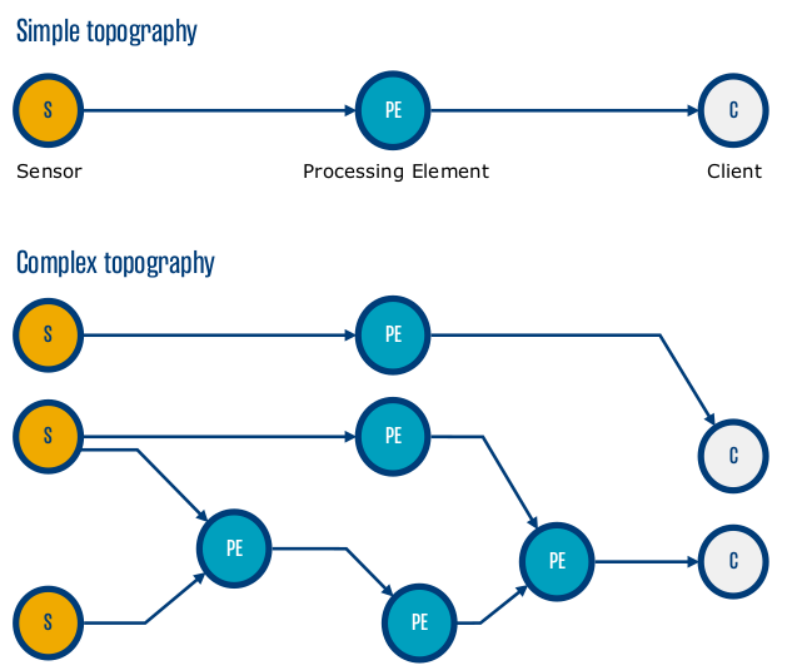

Figure 3.12: Two example topographies for stream processing systems.

Processing elements Processing elements are the main computational units of an SPS. They can have multiple functions, but at the very minimum they perform some operation on incoming data. If the SPS is distributed in nature, the processing elements can be implemented on separate hardware, which provides for load balancing throughout the system. This is beneficial, as processing of signals with high sampling rates and high channel counts can be computationally intensive. Processing elements may also have internal buffers for the short-term storage of data.

Clients The term 'client' describes any external application utilising information produced by the SPS. Clients can be considered end points (or sinks) in the SPS workflow. Some BCI frameworks consider the user of the system to be part of the client component.

In addition to the basic components of an SPS, there are various properties that are intrinsic to most such systems. These include scalability, distributed processing, load management, fault tolerance, latency management, and service discovery. Scalability refers to the ability to 
extend the system to include more sensors and processing elements (scaling methods suitable for very large systems are discussed by Jain et al. (2006)). In distributed processing, different parts of the system can run on different hardware, possibly even in different (geographical) locations. Optimisation strategies for distributed SPSs are presented by Golab and Özsu (2003). The SPS must also apply some method of controlling multiple concurrent queries from multiple clients. The usual implementation is some form of load balancer (for details of various load management methods and fault tolerance, see Abadi et al. $(2003,2005))$. Since the system is composed of multiple, distributed parts, latency management is important; that is, it must control or monitor the latency between components. Various methods for achieving low-latency real-time operation exist in both the Aurora and the S4 (Neumeyer et al., 2010) system. Finally, to enable communication between different system components, automated discovery of various sources and processing elements should be part of the system. For instance, metadata-based identification and discovery of system components were used by Aberer et al. (2007).

When one is fusing information from multiple signals for classification, it is important to consider the different time scales at which the signals operate too, as is noted by Hogervorst et al. (2014). The robustness of index determination over time, as discussed by, for example, Estepp and Christensen (2011), is an important issue, since the performance of the classifier degrades as time since calibration increases. Therefore, an online system should preferably incorporate some kind of automatic continuous calibration procedure. Further design considerations, requirements, and guidelines for implementing SPSs are presented by Balazinska (2005); Stonebraker et al. (2005); Cherniack et al. (2003).

\section{Software solutions for online analysis of signals}

Several stream processing software solutions exist that are capable of gleaning knowledge about a user's cognitive state from biosignals. The various stream processing systems intended for psychophysiology are all similar in design, possessing the elements outlined in the previous 
section. There are, however, some differences with regard to what types of signals the tools primarily support and what constitutes their end-use purpose. We will briefly review some of the available stream processing systems intended for physiological data. The software covered in this section is presented in Table 3.3.

Table 3.3: Existing stream processing systems for physiological data

\begin{tabular}{lll} 
Software & Reference & URL \\
\hline BCI2000 & Schalk et al. (2004) & $\begin{array}{l}\text { http://www.schalklab.org/research/ } \\
\text { bci2000 }\end{array}$ \\
& & http://openvibe.inria.fr/ \\
OpenViBE & Renard et al. (2010) & http://hcm-lab.de/projects/ssi/ \\
SSI & Wagner et al. (2011b) & http://sccn.ucsd.edu/wiki/BCILAB \\
BCILAB & Kothe and Makeig (2013) & https://github.com/bbci/wyrm \\
Wyrm & & https://github.com/bwrc/midas \\
MIDAS & Henelius and Torniainen &
\end{tabular}

Most of the SPS software packages available for physiological data are BCI and biofeedback frameworks. The BCI2000 package, for instance, is a full software solution tailored for BCI research. The design of BCI2000 is modular, cross-platform, and able to be extended with $\mathrm{C}++$. OpenViBE is another cross-platform BCI solution. It is intended for biofeedback and neurofeedback and can be extended through $\mathrm{C}++$ but also supports Python/Lua scripting. The SSI solution is a more versatile framework, suitable for processing a wide range of signals, from biosignals to video and audio. It includes functionality for feature extraction and classification. Extending SSI is handled with $\mathrm{C}++$. A BCI toolbox for MATLAB, BCILAB utilises the lab streaming layer protocol and provides a vast number of functions for analysing and classifying brain signals. Finally, the Python-based Wyrm is a more programming-oriented approach for implementing BCI set-ups.

Recently, the MIDAS framework, developed for the online processing of signals, was introduced (by the authors of this section). The goal with MIDAS is to provide a cross-platform, generic framework that is easy to use and extend. While the MIDAS solution utilises LSL for 
signal input, it provides only the framework for constructing modular and distributed analysis systems. In other words, MIDAS contains only the building blocks for an SPS, and it is up to the user to implement the necessary analysis modules. The MIDAS framework is written entirely in Python and can be easily extended. Communication with clients takes place over a REST API. This makes it easy to integrate psychophysiological indices extracted online by means of MIDAS with other applications.

It should be noted that BCI2000, OpenViBE, BCILAB, and SSI all can be set up and used without programming, since they include user-friendly tools for constructing workflows for BCI experiments. One key philosophy behind these software packages is to enable nonprogrammers to implement $\mathrm{BCI} /$ neurofeedback systems. In contrast, BCI systems implemented with the Wyrm toolbox or MIDAS require more programming and technical understanding.

MIDAS and BCI2000 are the only stream processing systems reviewed here that support a distributed design. This attribute can be useful in processing tasks that involve multiple input signals and large volumes of data.

\section{Hardware solutions for integrated online feature extraction}

In addition to software solutions for real-time signal fusion, there are dedicated hardware solutions for monitoring various aspects of a user's cognitive state; see Table 3.4.

For instance, the LifeShirt system is used to monitor the physiological signals (such as heart and respiration rate) of firefighters (Coca et al., 2009). Another example of hardware-based stream processing systems is the BioHarness 3, from Zephyr. Affectiva (founded by affective computing researcher Rosalind Picard) develops systems for online emotion recognition, especially using webcams (such as one called Affdex). Details of an interesting solution for monitoring heart rate (another application that uses a webcam) have recently been published (Poh et al., 2010).

Hardware solutions for monitoring cognitive states by means of EEG have been developed by Quasar and B-Alert. In addition, several 
Table 3.4: Examples of hardware that performs online signal analysis

\begin{tabular}{ll} 
Product and producer & Reference and/or URL \\
\hline LifeShirt & Coca et al. (2009) \\
& http://vivonoetics.com/products/sensors/ \\
& lifeshirt \\
& http://www.zephyranywhere.com/products/ \\
& bioharness-3 \\
BioHarness 3 & http://www.affectiva.com/ \\
& http://www.quasarusa.com/technology_ \\
Affectiva & applications.htm \\
Quasar & http://www.advancedbrainmonitoring.com/eeg- \\
& based-metrics \\
B-Alert & http://jins-meme.com/ \\
& http://ww.optalert.com/ \\
JIN CO., JINS-MEME & http://www.lumeway.com/ \\
Optalert & Guerreiro et al. (2013), http://www.bitalino.com/ \\
LumeWay & https://www.cooking-hacks.com/ehealth- \\
Bitalino-board & sensors-complete-kit-biometric-medical- \\
e-Health Sensor Platform & arduino-raspberry-pi
\end{tabular}

solutions are available for tracking fatigue on the basis of eye tracking. Examples include JINS-MEME, Optalert, and LumeWay.

Alongside application-specific products, there are platforms that allow a do-it-yourself approach to fusion of multiple biosensors. At the time of writing, the most noteworthy examples are the Bitalino-board (Guerreiro et al., 2013) and the e-Health Sensor Platform. Both feature numerous sensors (for EMG, EDA, BP, temperature, etc.) that can be connected to low-level microcontroller devices (e.g., an Arduino) or more high-level devices with compatible input/output connections (e.g., a Raspberry Pi). Both sets are highly configurable, with the tradeoff that some expertise is required for building and operating systems on these platforms.

\section{Conclusion}

The primary goal with online processing in HCI is to allow index determination to be used as a control signal for other components. Fusing information from multiple biosignals makes it possible to utilise complementary information and hence increase the robustness of the system. 
Achieving data fusion in real time is a challenging and computationally intensive task. However, that task can be addressed by utilising stream processing systems, such as those we have reviewed here. 
4

Overview and application

The 12 sections above on the state of the art have brought us to the point of understanding the nature of and application possibilities for a broad range of psychophysiological signals and topics. For continuing from this point, advice on practical application is given in sections 4.1 and 4.2. Before that, we present a reference to psychophysiological indices in HCI, in Table 4.1, for readers to be thoroughly prepared to plan their psychophysiological work with the aid of later sections.

Chapter 3 considered many examples of how one or more physiological signals can be used to index a psychological variable, a cognitive state, or an affect. Often, indices are more reliable and/or interpretable when additional variables are used to model the context, such as user behaviour in a computer interface. Table 4.1 is a synthesis covering several studies that have classified each index, with the relevant section describing the work done (where applicable). This general reference should assist the reader in performing a targeted literature review when working with one or more indices. 


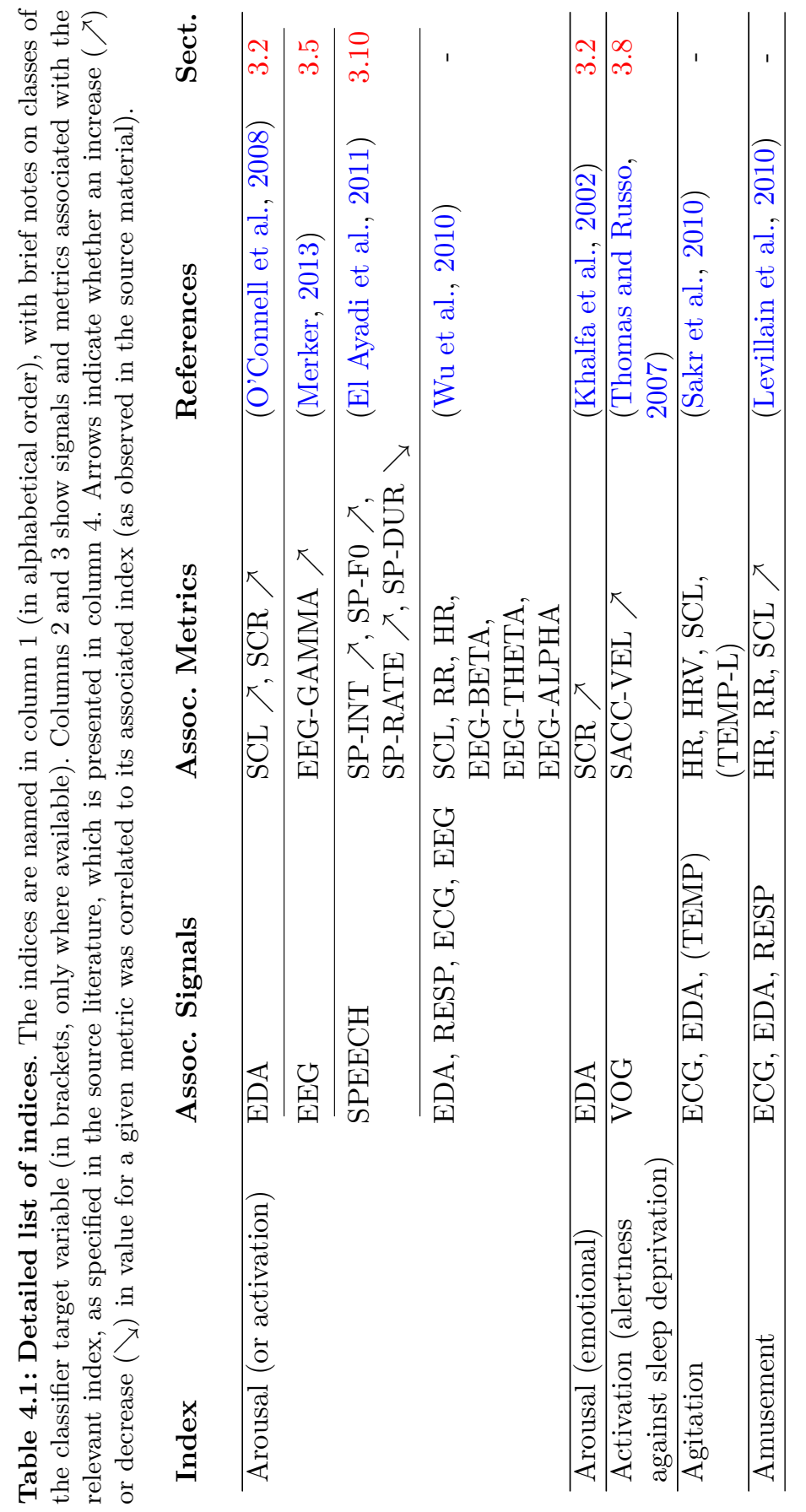




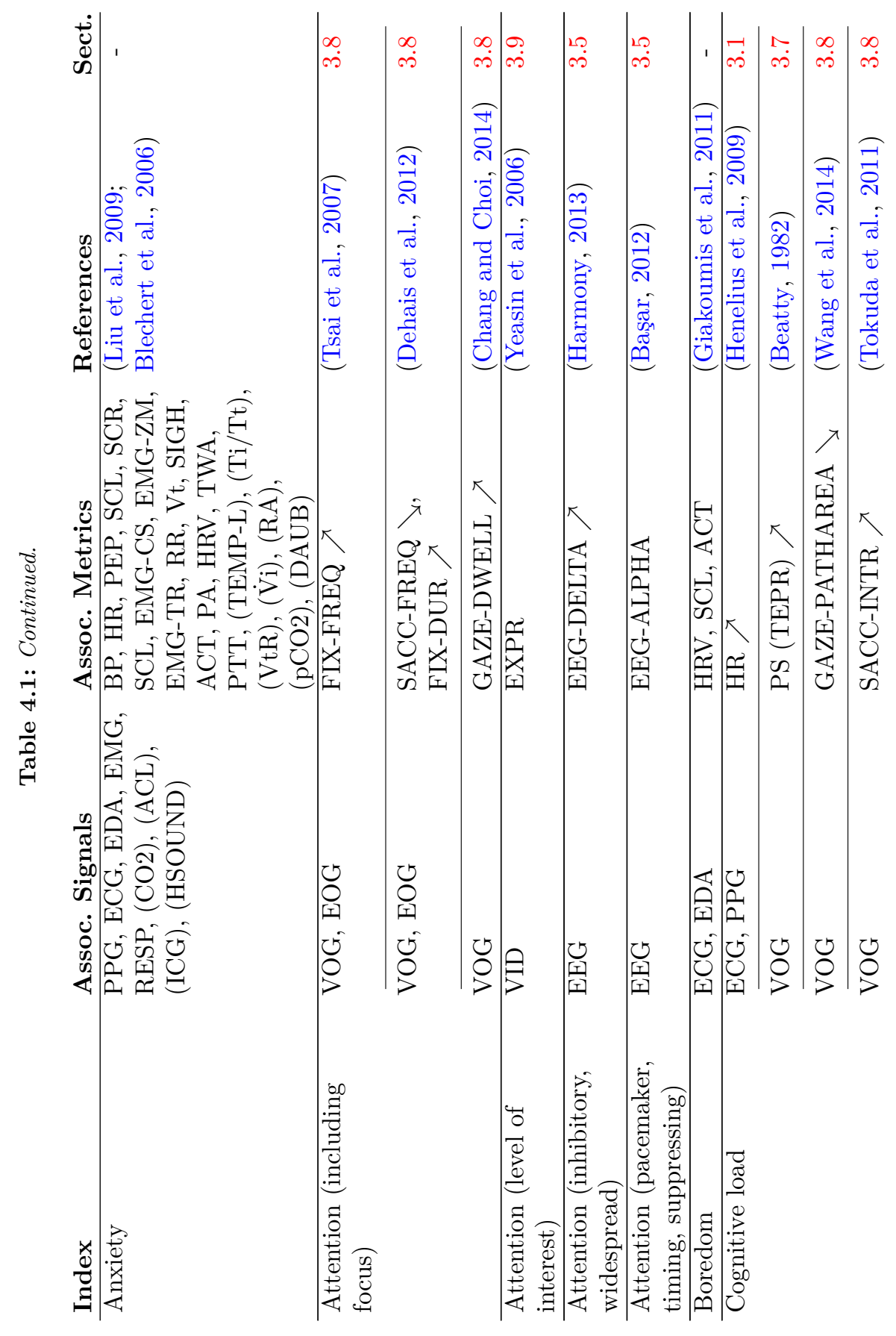




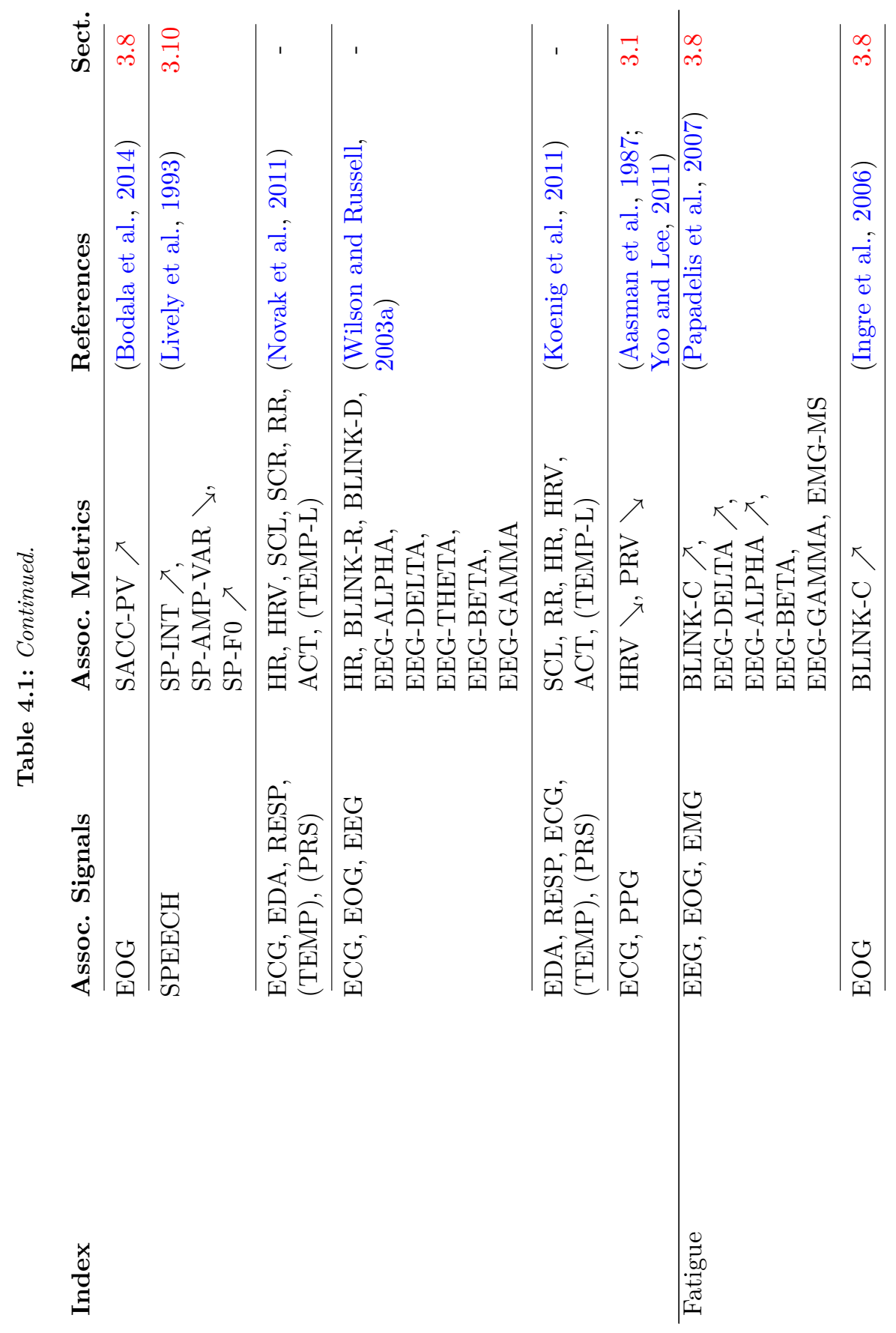




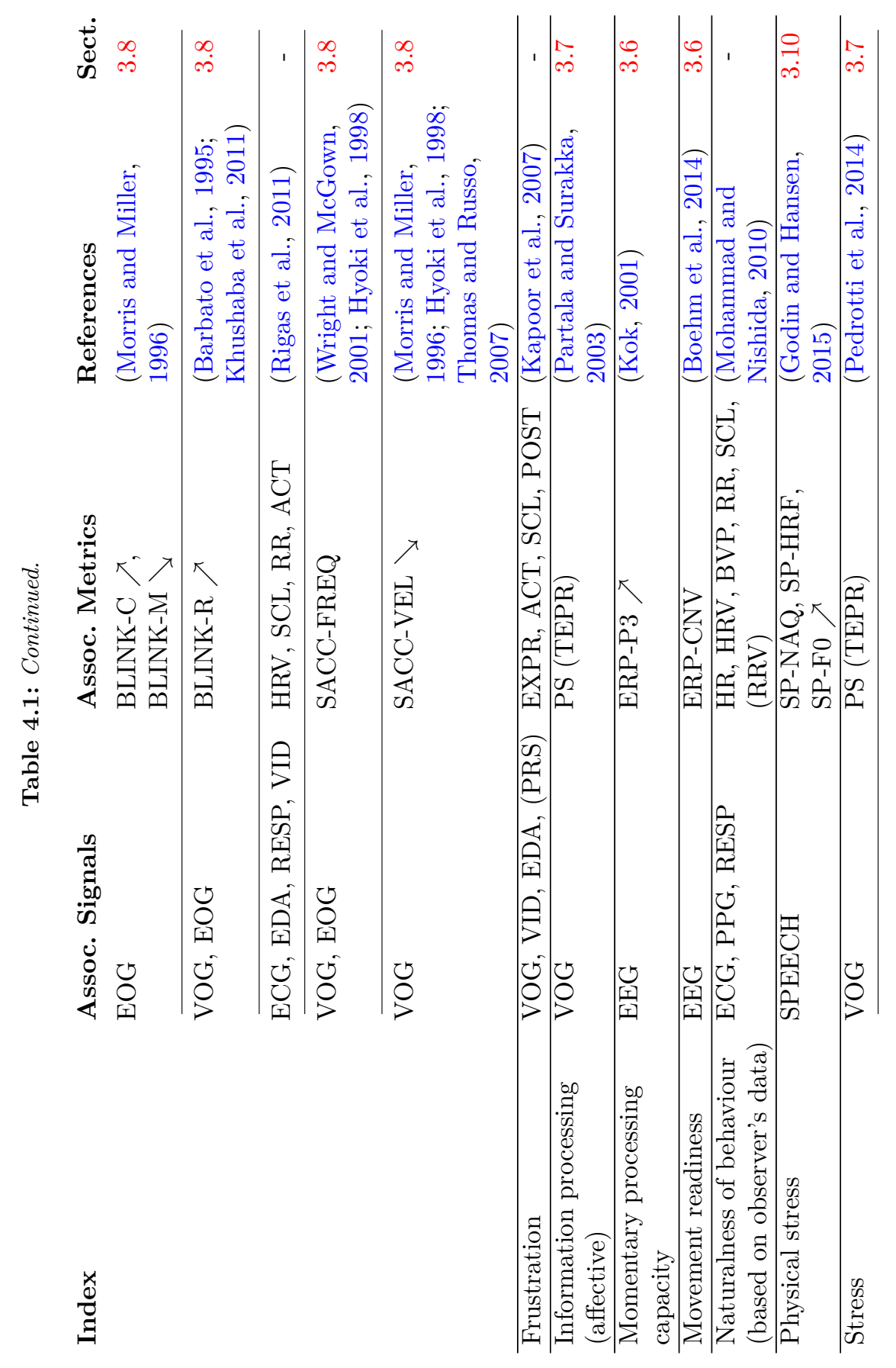




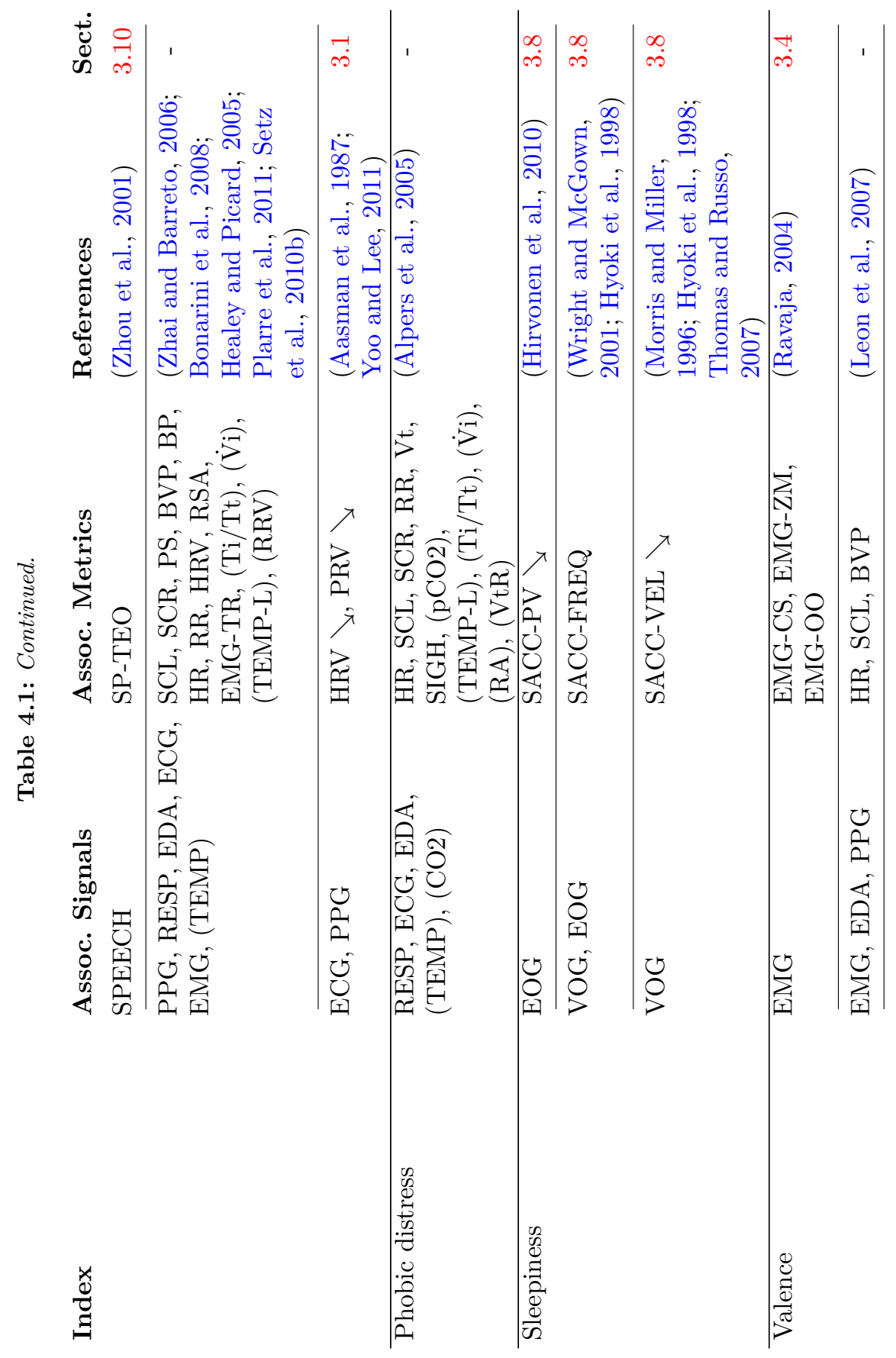




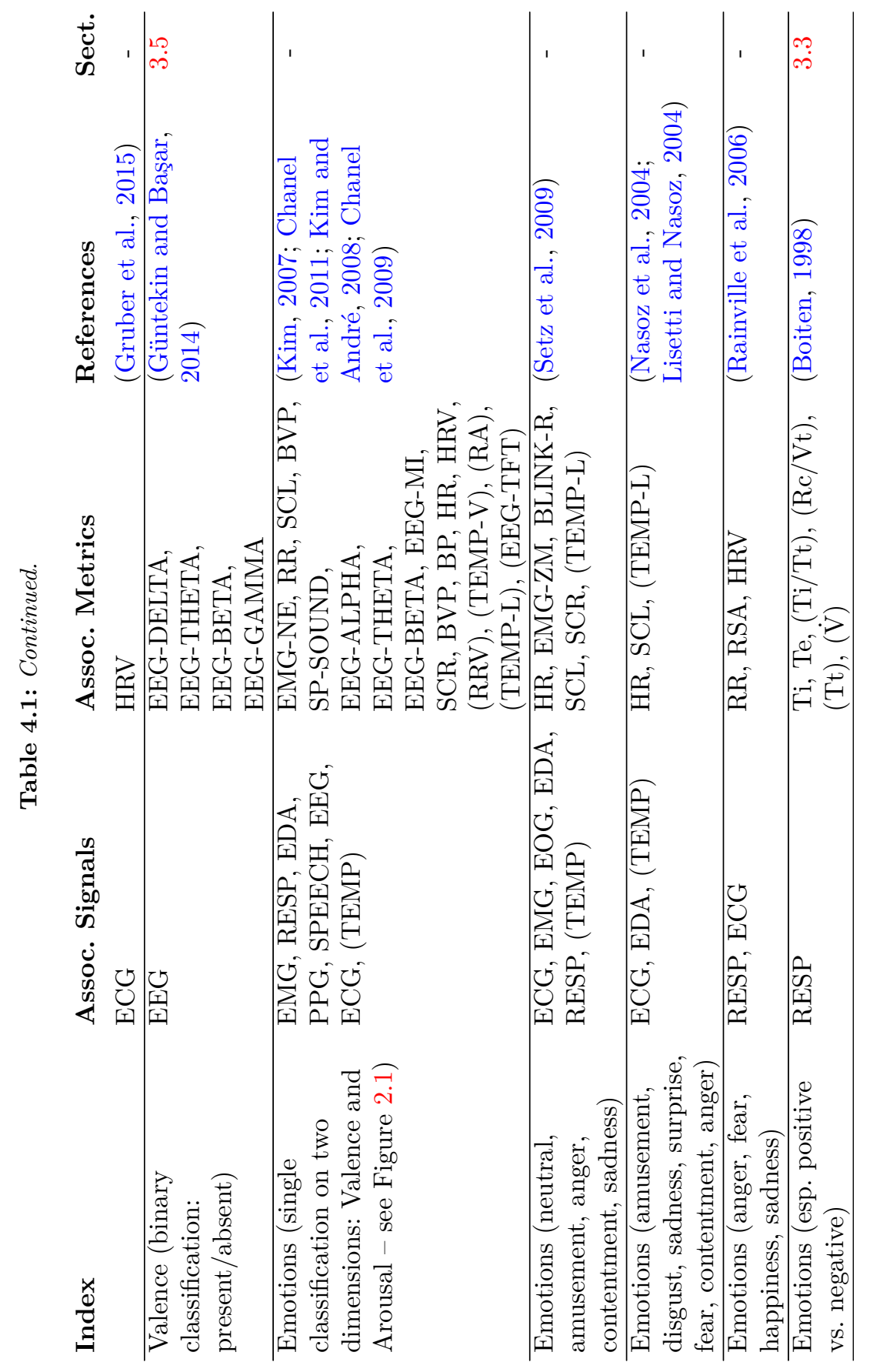









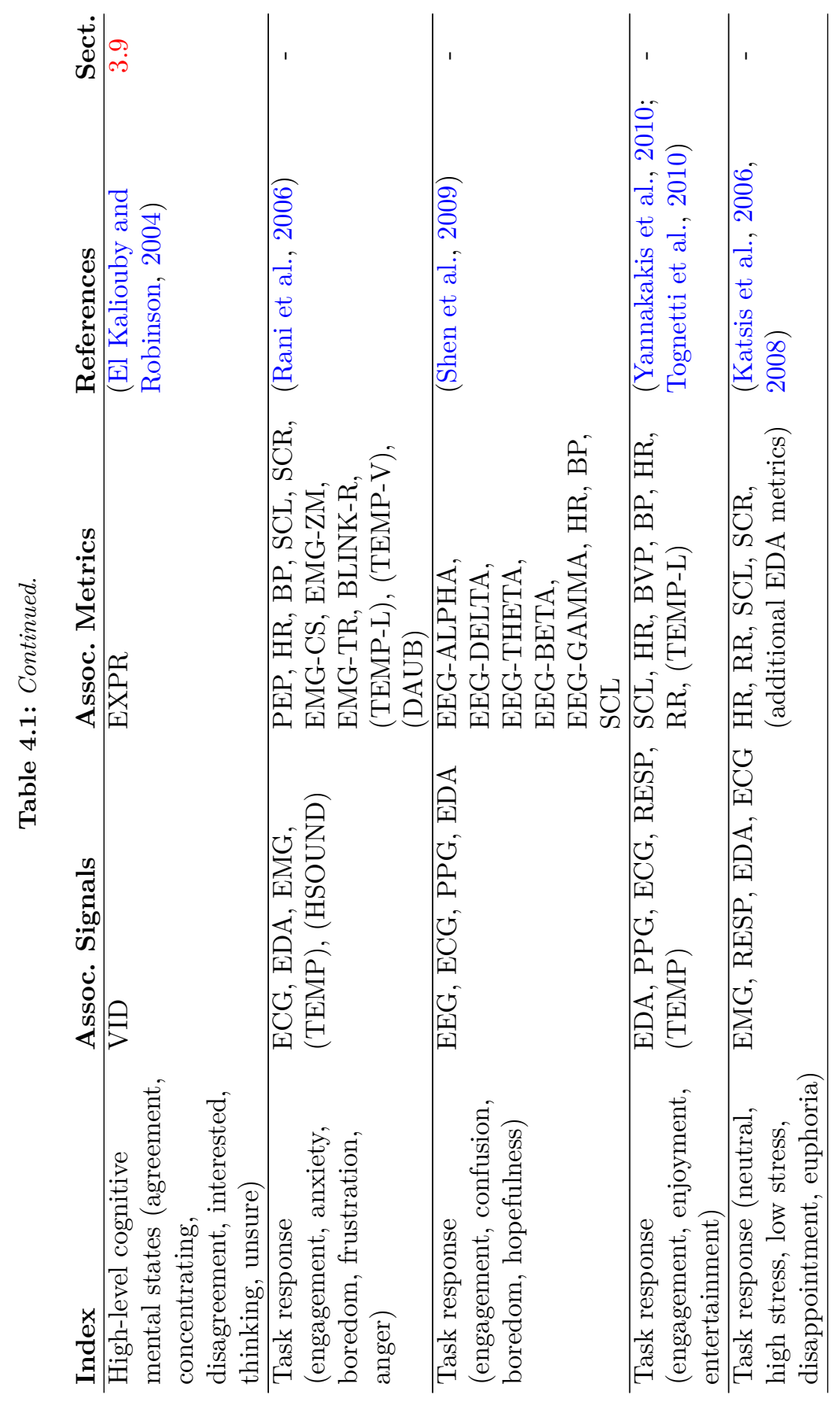




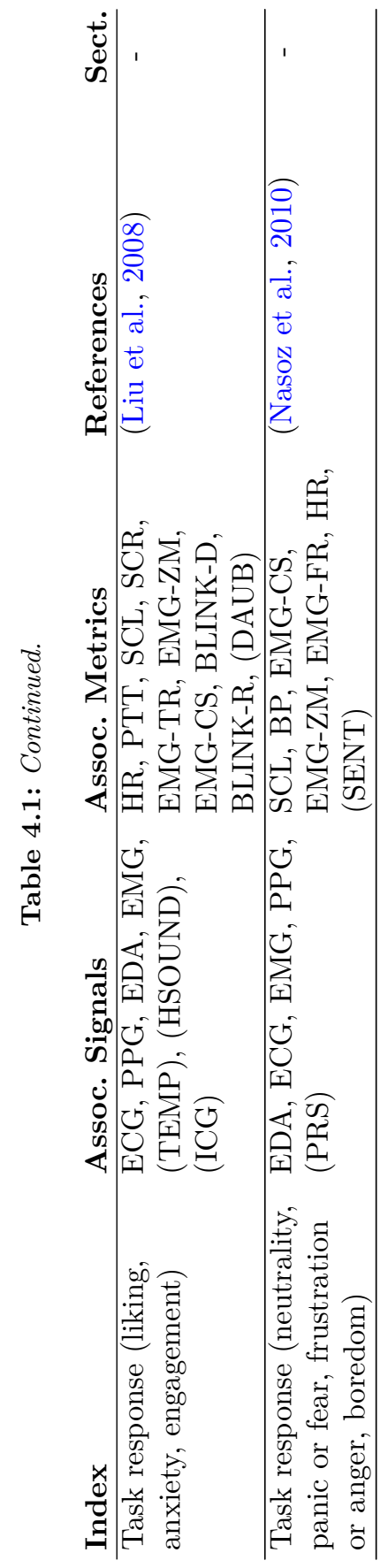




\subsection{Application areas}

A practitioner's purpose in using psychophysiology tends to fall into one of the four categories outlined below. In practice to date, outside specialised clinical uses, the most biosignal applications have been in the realm of classification, slightly fewer in that of prediction.

1. Among other applications, biosignals have been used for classification of a subject's psychological state; the task being performed, from among a set of predefined tasks; or trait-like features such as a depressive tendency (Weinberg et al., 2016).

2. The second most common area of application is prediction of behaviours, by learning a model of behaviour-associated physiology. Data can come from either a group of other individuals (in a between-subjects scenario) or a 'user model' of prior recordings (in a within-subjects design). Another prediction option is imminent concept drift/shift (Black and Hickey, 1999).

3. Biofeedback can be used in setting up, for example, an explicit control loop, psychological reinforcement directed toward desired states, navigation of information spaces, or clustering information presentation that is based on self-similarity of evoked physiology.

4. Entrainment may be applied to influence physiology toward desired states without biofeedback - for example, through rhythmic audio stimuli.

Classification The canonical use case is classification of some psychological state in line with biosignals. It is most often performed in a basic research setting. While this work would seem straightforward, there is a large amount of hidden complexity, in elements ranging from signal processing (see the discussion on feature extraction in section 3.2) to establishing psychological 'ground truth' by choosing some model of cognitive/affective state. For example, using the circumplex model of emotion, as described in section 3.2, is only one of many options (see chapter 5, below). 
It is clear from the foregoing discussion that, the maturity of the field notwithstanding, there is still a wealth of open questions. For just one example, we can consider EDA. Whilst it provides a robust, responsive, and feature-rich measure of autonomic arousal, many studies of EDA have used a model with only a single dimension, of low to high arousal, recorded from a single hand. One possible future improvement would be to link any feature of EDA to specific cognitive states. It is known that the CNS origins of EDA are multi-level and complex, with both ipsilateral and contralateral influences (Dawson et al., 2000), and that sympathetic arousal is not always symmetric across the body (Picard et al., 2015). If a novel EDA metric were established that considers the difference between right- and left-hand EDA features, hemispheric asymmetry in the brain might be determined to be responsible for the signal differential. Accordingly, cognitive indications could be compassed. Although highly speculative, this example illustrates well the untapped potential of an area of study that is more than 100 years old, and similar issues exist for most other signals.

Moving beyond basic research to explore applications is the true cutting edge. For example, cardiovascular metrics are already fairly well established as indicators of, for example, mental workload (see section 3.1). However, such classifiers tend to require offline analysis, especially on account of the problem of accounting for variation stemming from the context; accordingly, using ECG in an online manner as a control signal in physiology-responsive systems remains problematic.

Prediction Predicting future behaviour, whether between or within subjects, is a large research area with ramifications for work in such fields as operator safety, online recommendations, and games. Using biosignals for this purpose is rendered difficult by their inherent noise. At present, brain-computer interface technology is being developed primarily to aid incapacitated individuals. The system must predict the user's intentions on the basis of an EEG signal for appropriate control of a system such as a wheelchair; however, in most real-world conditions, this is a very difficult task, as EEG signals are noisy and multidimensional. Another possible use of predicting changes in a user's state is 
in helping to address 'concept drift', wherein change over time invalidates existing classifications. For example, classification of autonomic arousal can be sensitive to ambient temperature, as the user's thermoregulatory sweating alters the tonic conductance level. A system with predictive capacity can adapt to this change by updating its classifier.

Biofeedback Biofeedback is an established clinical application but also is seeing increasing popularity in consumer products. For example, respiration is partly conscious and partly unconscious; it thereby exemplifies in one signal the concepts of implicit and explicit biofeedback - i.e., feedback coming from signals outside or within conscious control. Breathing techniques have been used over much of recorded history as an aid to self-control of body and mind. Hence, studying the explicit/implicit use of respiration can aid in illustrating how the balance between explicit and implicit feedback is handled in signals that are less easy to control.

Entrainment Entrainment is the least mature of the four areas and hence the least application-ready. Research in this area points to the possibility of cognitive enhancement via physiological entrainment (Reedijk et al., 2015). Individual-to-individual differences in biological characteristics relevant in respect of cognition are one modulating factor that must be accounted for. This is a thorny issue, because usually which particular individual difference is relevant is far from unambiguous.

Summary Though these areas of application are conceptually fundamental, the practice in each of them is going to be radically altered by novel technology in sensors and signal processing, driven by miniaturisation, remote sensing, and constantly increasing computation power and algorithmic sophistication. For example, remote methods have recently been demonstrated as able to measure heart rate in the absence of physical contact, by such means as video (see section 3.9, above) or radar (Ren et al., 2015; Sakamoto et al., 2015). Remote sensors can, in turn, be connected directly to high-performance computers, a 
combination that creates possibilities for real-time analyses of the sort previously limited to offline application.

\subsection{Practical guidelines}

As chapter 3 makes clear, numerous signals and devices are available, and the development of novel measurement and analysis methods is advancing rapidly. While on the surface it appears as if almost everything is possible, with a large proportion of the current solutions there are specific limitations to applicability due to technical, physiological, and model-dependent demands.

To arrive at valid, reproducible results, one should possess a sound theoretical understanding as to where and how a given psychophysiological signal originates, how the physiological signal behaves under variable conditions, and the way in which the index in question is derived from the signal. In the literature on applied integration of psychophysiology and HCI, there is a tendency to rely on a specific commercial device manufacturer's methods in calculating an index for factors such as stress, without any consideration of methodology. Before making a selection, the practitioner should understand the signal origin and the underlying psychophysiological model/mechanisms. The constraints and limitations these impose on the relevant use case must be borne in mind.

In this section of the primer, we guide the reader through a practical process of signal source selection for psychophysiological indices. From chapter 3 , the reader will be aware of the large number of options for implementing the application in question. A simple process is required for handling this complexity. The process applied should comprise at least the following steps, as illustrated in Figure 4.1.

1. Select the desired indices for physiological phenomena

Table 4.1 provides a starting point for mapping out the possible options.

For example, if you are interested in measuring stress and physical strain in operation of a large touchscreen interface for stock 


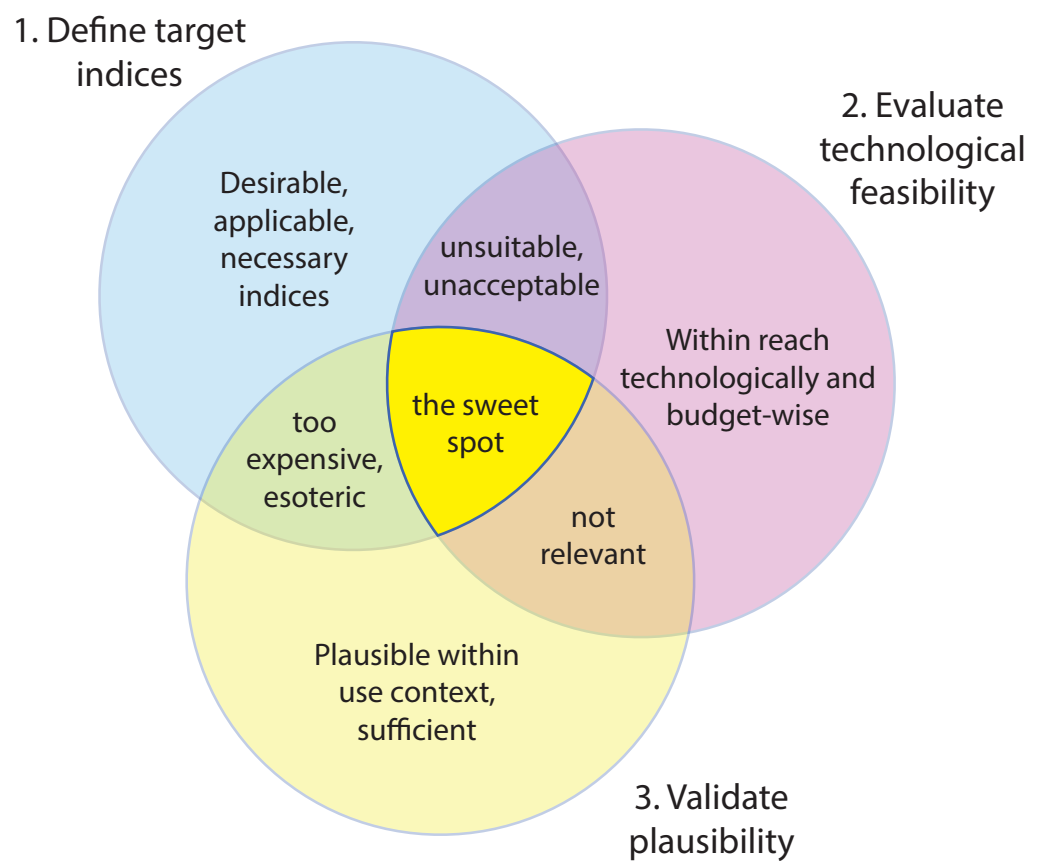

Figure 4.1: Choosing candidate signals.

exchange data, various indices may be suitable. Questionnaire data show that the operators experience time pressure and worry about making the right decisions. Additionally, in working with this large touchscreen interface, they have to hold their hands up, facing the screen, and then make large movement gestures in order to operate the interface.

2. Map out psychophysiological metrics within reach, for use in your system, and consider their parameters

Which physiological phenomena have been shown to indicate the target internal state? What is the temporal resolution of the signal, what physiological lags are present, and how well do these dovetail with the requirements of the system being developed? What is the 'resolution' of the signal - is it suitable for indicating a continuous change or providing a binary classification 
between two states? Which of the possible choices serves as a credible technical solution for the application? Again, Tables 2.2 and 4.1 can assist you in the selection of candidate metrics.

In the touchscreen example, you might select heart rate metrics, EDA, and speech analysis as the stress signal candidates and choose surface-EMG measurements as a candidate for measuring activity with the touch-based interface and the resulting physiological strain on the upper torso muscles. You are likely to find that all of these candidates (ECG, EDA, speech-recording microphones, and sEMG) can be addressed by existing compact wearable devices, although each of them comes from a separate manufacturer and, hence, using all of them would mean having the user wear a large set of measurement devices.

3. Validate the plausibility of the selected measurement methods in the use scenario

The next step is to consider the circumstances of use: Does the subject move extensively or instead stay put at a desk? Is the environment noisy or perhaps exposed to direct sunlight, with potential to affect the measurements? What kind of equipment is the user able (or willing) to wear? What kind of instrumentation might affect the user's ability to operate the equipment used in his or her work? Are there environmental disturbances such as sources of electrical interference, vibration, or extreme temperatures? Finally, how long does the equipment take to set up, and is it going to need periodic (re-)calibration?

Continuing with the example above, you may find from analysis of the operators' daily behaviour at the screen that the subject is constantly moving, implying that stress metrics based on heart rate variability would likely be confounded by changes in heart function that arise from physical activity. On the other hand, this scenario speaks in favour of applying ECG, sEMG, and/or three-dimensional actigraphy for physical strain measurements. With this continual movement not being great enough to make the operator sweat, EDA might still prove a viable signal source 
for analysing stress. The operator in the scenario may also speak regularly with colleagues in the same room and over the phone, wearing a microphone for phone calls. This gives you the opportunity to record speech utterances in high quality as a natural part of the work without having to fabricate subtasks designed to generate speech for research purposes.

After working through steps 1-3, you should have a compact list of candidate signals for indicating psychophysiological phenomena. For a more in-depth application that involves implementing a software client that takes physiological signals as inputs, the following issues should be considered too:

- How will you implement the interface of the measurement device(s) for accessing data (online)? Does the device manufacturer offer access to the raw data stream or instead a pre-calculated, proprietary index? Does the device support an open-access interface or provide a well-documented application programming interface (API), used for interfacing new code with a system's existing features)? See section 3.12 for details on this issue.

- Will you be able to create a multimodal set-up? This should increase redundancy, allow you to correlate information across signals in order to improve the robustness and sensitivity of your set-up, and possibly aid in disambiguating overlapping analysis results. Section 3.11 can guide you further in this respect.

- What is the temporal nature of the input - with what frequency do changes in the signal represent meaningful changes in the underlying state of the subject? For example, a physiological signal indicating drowsiness (exhibiting slow change) could be an input that causes the UI to be more stimulating or is used by a machine recommender system to deliver fewer recommendations, whereas a signal indicating interest (exhibiting rapid change) could prompt highlighting individual knowledge units as more relevant. See Table 2.2 for estimates of each signal's temporal frame. 
- You should consider the significance of the input: How fundamental are changes in the signal to the state of the user? How can you validate against a ground truth? How sensitive is the signal to a user's state changes, and how ambiguous are those changes? In what ways does inherent measurement noise affect validity? What is the resolution of a relevant change in the signal level, and how can that be mapped to functionality on the application side?

- You should investigate the reliability and robustness of the signal and possible countermeasures to address noise and other external effects. What are the possible sources of noise (in the relevant use scenario), and how can they be countered? Should the strap be tightened, or, in contexts such as laboratory research, should you use a conducting gel for obtaining better contact for the measurement?

- There may well be inter-individual variability in signal quality. What kinds of differences do individuals exhibit with respect to the physiological phenomenon that is being measured? For instance, resting heart rate might vary by $20 \%$ between subjects. How do differences in physiology between individuals, such as body shape or amount of hair, influence the signal? 


\section{5}

\section{Concluding remarks}

\section{Challenges}

Physiology in HCI is emerging as an important area for study, as is attested by journal special issues, books, and conference sessions that explore applications in everyday situations beyond sports and clinical settings. Diverse technological, methodological, and theoretical challenges therefore exist in this regard. For assurance of deployability and acceptance by the user, there must be improvements in sensor robustness, sensors' calibration, miniaturisation, and integration into ergonomically designed and unobtrusive products. Robustness and reliability in identifying and recognising physiological states remains an unresolved problem. Moreover, many psychophysiological metrics rely on sources that change over time, thereby creating difficulties in user calibration.

Research in physiology is dispersed across numerous fields and is often difficult to build upon or compare with previous work. Addressing this issue is going to require efforts devoted to standardisation of methods and procedures, in order to provide established results that can inform development of solutions. Often, the studies reported upon 
use ad hoc machine learning methods of various types, which are difficult to replicate. In particular, there is a dearth of standard tools, equipment, and specifications that could serve building of systems and applications in HCI, because, when choosing a device, developers are confronted with a vast range of devices that have various degrees of validation and many proprietary APIs.

The advantage brought by rapidly improving technology (see section 4.1, above) also poses challenges. One drawback of employing novel methods is that they might generate noisier signals (this is especially true of remote sensing, as exemplified by radar HR) or more complex data (in particular, with novel hardware, as in skin imaging). Therefore, metric extraction algorithms, in turn, need to increase in complexity if they are to generate meaningful indices.

A key challenge is that physiological responses cannot precisely and uniquely identify a source, and a given signal may be correlated with multiple psychophysiological states or phenomena. Research should move beyond investigating how physiology is linked to certain levels of any one state (low or high arousal, for instance) to examine how physiology is linked to multiple states.

Besides the more established indices listed in Table 4.1, there are numerous psychological states and phenomena that are starting to be investigated in earnest, so that applications that bring added value to users in everyday life can be developed. Examples of novel indices include relevance in information retrieval (Eugster et al., 2014; Barral et al., 2015), flow in games (Nacke and Lindley, 2008), mindfulness related to HRV (Burg et al., 2012), derivatives of social presence in a mediated virtual environment (Spapé et al., 2013), and mediated touch through haptic feedback (Spapé et al., 2015). The emerging interest in many, quite different psychophysiological phenomena and indices raises the issue of investigating which of these are independent or instead can be explained in terms of existing indices.

One of the challenges beyond those of signal analysis is the problem of establishing consensus on the underlying theories that govern or link the psychological phenomena to physiology. Emotions are a good example. There is still debate on their definition, exemplified by the 
argument over whether facial expressions are an accurate reflection of internal emotion (Fridlund, 1991; Hess et al., 1995) (see also the discussion in section 3.4, above). The competing theories have resulted in creation of several, very different models, such as the PAD circumplex of emotion (Posner et al., 2005) (see Figure 2.1, above), the PANAS model (Watson et al., 1988; Mehrabian, 1997), and the basic Ekmanian emotions (Ekman and Davidson, 1994).

Another example that can be cited is arousal, which is often addressed with a uni-dimensional, bipolar model. This is clearly a gross simplification. Stress is one commonly studied index related to arousal, and it alone comes in multiple flavours even when one considers only the difference between positive eustress and negative distress. Furthermore, as mentioned above in section 3.2, arousal can be described not just in terms of affect but also in connection with effort and preparatory activation. Similar complexity is characteristic of any other aspect of psychophysiology. This supports the assertion that the average practitioner, who is more interested in applications than in basic research, stands to benefit from selecting and thoroughly understanding a single validated model and approach for the problem at hand.

Recent advances in machine learning allow one to develop systems linking signals and higher-level states through black boxes, particularly through deep learning approaches. While such approaches do provide a solution in certain cases, the true challenge is to use machine learning in support of theory development, so that causal models can be identified and, thereby, more transferable knowledge can be gleaned.

\section{Conclusion}

The application of psychophysiology to HCI is complicated by the extreme breadth and depth of topics and also by the great complexity of parameter choices and design decisions for algorithms and hardware. We have addressed this complexity by a) focusing on practical aspects and $b$ ) introducing the topics in an order roughly corresponding to the depth of the topics and breadth of their range (from the standpoint of application). Throughout the work, we have aimed for a tight focus on 
the function of a primer. No effort has been made to delve into issues such as privacy, compliance, or ethics - in part because such issues are covered elsewhere (Fairclough, 2009a; Ajunwa et al., 2016).

Narrative-style reviews are inexhaustive and non-systematic by nature. Therefore, they are susceptible to bias. The method by which we compiled Tables 2.1, 2.2, and 4.1 is systematic and replicable (see chapter 2). Nonetheless, we have deliberately avoided meta-analysis, because a) the number of such analyses needed is very high $(N=$ at least the number of indices in Table 4.1) and b) every one of these analyses requires a detailed report, which should be accessible as a standalone reference text and, accordingly, is a subject for future work. Such work could take the form of a systematic review of the measurement properties of a psychophysiological index; for example, the properties of mental workload. See Vet et al. (2011) for a discussion of this approach. Finally, quantification of results also necessarily becomes dated, while this primer is intended to serve as a relatively stable reference text.

The medical domain shows great success at answering clearly defined research questions through systematic review - a task with which psychophysiology for health applications or HCI has not yet engaged. So long as there remains a lack of systematically derived guidelines with broad coverage, there is need for a 'quick and dirty' guide to the field such as this, to serve as an introduction and reference point for non-specialists. The paper addresses said need in a manner that is modular and very broad in scope, so that the reader can obtain a clear picture of the entire field yet also focus directly on implementing useful applications. 


\section{Acknowledgments}

The work was supported in part by the Finnish Funding Agency for

Innovation (project Re:Know, No. 5159/31/2014). The authors wish to thank our editors and peer reviewers, and proofreader Anna Shefl.

BC co-ordinated contributions; designed the paper structure; acted as internal editor; wrote chapters 1 and 2 and sections 3.2 and 4.1; contributed to sections $3.3,3.4,3.5,3.6$; and peer-reviewed sections 3.5 and 3.9 .

MF contributed to the internal editing; wrote section 3.11; compiled Tables 2.1, 2.2, and 4.1; and peer-reviewed section 3.12.

KL contributed to the internal editing; wrote sections $3.8,3.10$, and 4.2; and peer-reviewed section 3.7.

JT contributed to the internal editing; wrote section 3.12 ; contributed to sections $3.2,3.4$; and peer-reviewed section 3.11 .

$\mathrm{AH}$ wrote section 3.1; contributed to section 3.12; and peer-reviewed section 3.3 .

LA wrote section 3.5; contributed to section 3.6; and peer-reviewed section 3.2 .

OB wrote section 3.7 and peer-reviewed section 3.8.

IK wrote section 3.3 and peer-reviewed section 3.1 .

TV wrote section 3.9 and peer-reviewed section 3.10 .

MH wrote section 3.6.

NR wrote section 3.4 and peer-reviewed section 3.6.

GJ acted as internal editor; wrote sections 1.1 and 4.3 ; and peerreviewed section 3.4 . 


\section{References}

Jans Aasman, Gijsbertus Mulder, and Lambertus J. M. Mulder. Operator effort and the measurement of heart-rate variability. Human Factors: The Journal of the Human Factors and Ergonomics Society, 29(2):161-170, 1987.

Daniel J. Abadi, Don Carney, Ugur Çetintemel, Mitch Cherniack, Christian Convey, Sangdon Lee, Michael Stonebraker, Nesime Tatbul, and Stan Zdonik. Aurora: A new model and architecture for data stream management. The VLDB Journal-The International Journal on Very Large Data Bases, 12(2):120-139, 2003.

Daniel J. Abadi, Yanif Ahmad, Magdalena Balazinska, Ugur Cetintemel, Mitch Cherniack, Jeong-Hyon Hwang, Wolfgang Lindner, Anurag Maskey, Alex Rasin, Esther Ryvkina, et al. The design of the Borealis stream processing engine. In CIDR, volume 5, pages 277-289, 2005.

Karl Aberer, Manfred Hauswirth, and Ali Salehi. Infrastructure for data processing in large-scale interconnected sensor networks. In Mobile Data Management, 2007 International Conference on, pages 198-205. IEEE, 2007.

Minkyu Ahn, Mijin Lee, Jinyoung Choi, and Sung Chan Jun. A Review of Brain-Computer Interface Games and an Opinion Survey from Researchers, Developers and Users. Sensors, 14(8):14601-14633, Aug. 2014.

Ifeoma Ajunwa, Kate Crawford, and Jason Schultz. Limitless Worker Surveillance. California Law Review, 105(3):in press, Mar. 2016.

D. M. Alexander, C. Trengove, P. Johnston, T. Cooper, J. P. August, and E. Gordon. Separating individual skin conductance responses in a short interstimulus-interval paradigm. Journal of Neuroscience Methods, 146(1): 116-23, July 2005. ISSN 0165-0270. 
Paavo Alku. Glottal inverse filtering analysis of human voice production-a review of estimation and parameterization methods of the glottal excitation and their applications. Sadhana, 36(5):623-650, 2011.

Jennifer Allanson and Stephen H. Fairclough. A research agenda for physiological computing. Interacting with Computers, 16(5):857-878, 2004.

John Allen. Photoplethysmography and its application in clinical physiological measurement. Physiological Measurement, 28(3):R1, 2007a.

John Allen. Photoplethysmography and its application in clinical physiological measurement. Physiological Measurement, 28(3):R1, 2007b.

Brendan Allison, Bernhard Graimann, and Axel Gräser. Why use a BCI if you are healthy. In Anton Nijholt and Desney Tan, editors, BRAINPLAY 07 Brain-Computer Interfaces and Games Workshop at ACE (Advances in Computer Entertainment), pages 7-11, Salzburg, Austria, 2007. ACE.

G. W. Alpers, F. H. Wilhelm, and W. T. Roth. Psychophysiological assessment during exposure in driving phobic patients. Journal of Abnormal Psychology, 114(1):126-139, 2005.

Shun Arai, Kazuhisa Ohira, Niwat Thepvilojanapong, Nobuji Tetsutani, Yoshito Tobe, Mayumi Oyama-Higa, and Yoshikatsu Ohta. A design of software adaptive to estimated user's mental state using pulse wave analysis. In Networked Sensing Systems (INSS), 2012 Ninth International Conference on, pages 1-4. IEEE, 2012.

Aymen A. Awad, Mohamed-Ashraf M. Ghobashy, Wagih Ouda, Robert G. Stout, David G. Silverman, and Kirk H. Shelley. Different responses of ear and finger pulse oximeter wave form to cold pressor test. Anesthesia $\&$ Analgesia, 92(6):1483-1486, 2001.

Richard Backs and Wolfram Boucsein. Engineering Psychophysiology Issues and Applications. Taylor \& Francis, Hoboken, 1st edition, 2000. ISBN 9781410605436.

Richard W. Backs, H. Ted Navidzadeh, and Xidong Xu. Cardiorespiratory indices of mental workload during simulated air traffic control. In Proceedings of the Human Factors and Ergonomics Society Annual Meeting, volume 44, pages 89-92. SAGE Publications, 2000.

Jeremy N. Bailenson, Emmanuel D. Pontikakis, Iris B. Mauss, James J. Gross, Maria E. Jabon, Cendri A. C. Hutcherson, Clifford Nass, and Oliver John. Real-time classification of evoked emotions using facial feature tracking and physiological responses. International Journal of Human-Computer Studies, 66(5):303-317, 2008. 
S. Baillet, J. C. Mosher, and R. M. Leahy. Electromagnetic brain mapping. IEEE Signal Processing Magazine, 18(6):14-30, Nov. 2001. ISSN 1053-5888.

Magdalena Balazinska. Fault-tolerance and load management in a distributed stream processing system. PhD thesis, MIT Computer Science and Artificial Intelligence Laboratory, 2005.

Elisabetta Baldi and Corrado Bucherelli. The inverted ' $u$-shaped' dose-effect relationships in learning and memory: Modulation of arousal and consolidation. Nonlinearity in Biology, Toxicology, Medicine, 3(1):9-21, 2005. ISSN 1540-1421.

Mahdi Bamdad, Homayoon Zarshenas, and Mohammad A. Auais. Application of BCI systems in neurorehabilitation: A scoping review. Disability and Rehabilitation: Assistive Technology, 10(5):355-364, Sep. 2015. ISSN 17483107.

Giuseppe Barbato, Gianluca Ficca, Michele Beatrice, Margherita Casiello, Giovanni Muscettola, and Franco Rinaldi. Effects of sleep deprivation on spontaneous eye blink rate and alpha eeg power. Biological Psychiatry, 38 (5):340-341, 1995.

Oswald Barral, Manuel J. A. Eugster, Tuukka Ruotsalo, Michiel M. Spapé, Ilkka Kosunen, Niklas Ravaja, Samuel Kaski, and Giulio Jacucci. Exploring peripheral physiology as a predictor of perceived relevance in information retrieval. In Proceedings of the 20th International Conference on Intelligent User Interfaces, pages 389-399. ACM, 2015.

Erol Başar. A review of alpha activity in integrative brain function: Fundamental physiology, sensory coding, cognition and pathology. International Journal of Psychophysiology, 86(1):1-24, Oct. 2012. ISSN 0167-8760.

Erol Başar, Canan Başar-Eroglu, Sirel Karakaş, and Martin Schürmann. Gamma, alpha, delta, and theta oscillations govern cognitive processes. International Journal of Psychophysiology, 39(2-3):241-248, Jan. 2001. ISSN 0167-8760.

Jessica D. Bayliss and Dana H. Ballard. Single trial P3 epoch recognition in a virtual environment. Neurocomputing, 32-33:637-642, Jun. 2000. ISSN 09252312.

Jackson Beatty. Task-evoked pupillary responses, processing load, and the structure of processing resources. Psychological Bulletin, 91(2):276, 1982.

Jackson Beatty and Brennis Lucero-Wagoner. The pupillary system. Handbook of Psychophysiology, 2:142-162, 2000. 
Mathias Benedek and Christian Kaernbach. A continuous measure of phasic electrodermal activity. Journal of Neuroscience Methods, 190(1):80-91, June 2010a. ISSN 1872-678X.

Mathias Benedek and Christian Kaernbach. Decomposition of skin conductance data by means of nonnegative deconvolution. Psychophysiology, 47 (4):647-658, 2010b.

Hans Berger. Über das Elektrenkephalogramm des Menschen. Archiv für Psychiatrie und Nervenkrankheiten, 87(1):527-570, Dec. 1929. ISSN 00039373, 1433-8491.

Natascia Bernacchia, Lorenzo Scalise, Luigi Casacanditella, Ilaria Ercoli, Paolo Marchionni, and Enrico Primo Tomasini. Non contact measurement of heart and respiration rates based on kinect ${ }^{\mathrm{TM}}$. In Medical Measurements and Applications (MeMeA), 2014 IEEE International Symposium on, pages 1-5. IEEE, 2014.

Gary G. Berntson. Heart rate variability: Origins, methods and and interpretive caveats. Psychophysiology, 34:623-648, 1997.

Alberto Betella, Riccardo Zucca, Ryszard Cetnarski, Alberto Greco, Antonio Lanatà, Daniele Mazzei, Alessandro Tognetti, Xerxes D. Arsiwalla, Pedro Omedas, Danilo De Rossi, and Paul F. M. J. Verschure. Inference of human affective states from psychophysiological measurements extracted under ecologically valid conditions. Frontiers in Neuroscience, 8:286, Jan. 2014. ISSN 1662-4548.

George E. Billman. The lf/hf ratio does not accurately measure cardiac sympatho-vagal balance. Heart Rate Variability: Clinical Applications and Interaction between HRV and Heart Rate, page 54, 2007.

Paola Binda, Maria Pereverzeva, and Scott O. Murray. Pupil size reflects the focus of feature-based attention. Journal of Neurophysiology, 112(12): 3046-3052, 2014. ISSN 0022-3077.

M. Black and R. J. Hickey. Maintaining the performance of a learned classifier under concept drift. Intelligent Data Analysis, 3(6):453-474, 1999.

Jens Blechert, Marta Lajtman, Tanja Michael, Jürgen Margraf, and Frank H. Wilhelm. Identifying anxiety states using broad sampling and advanced processing of peripheral physiological information. Biomedical Sciences Instrumentation, 42:136-141, 2006.

Terry D. Blumenthal, Bruce N. Cuthbert, Diane L. Filion, Steven Hackley, Ottmar V. Lipp, and Anton van Boxtel. Committee report: Guidelines for human startle eyeblink electromyographic studies. Psychophysiology, 42(1): 1-15, Jan. 2005. ISSN 0048-5772. 
I. P. Bodala, Yu Ke, H. Mir, N. V. Thakor, and H. Al-Nashash. Cognitive workload estimation due to vague visual stimuli using saccadic eye movements. In Engineering in Medicine and Biology Society (EMBC), 2014 36th Annual International Conference of the IEEE, pages 2993-2996, Aug. 2014.

Udo Boehm, Leendert van Maanen, Birte Forstmann, and Hedderik van Rijn. Trial-by-trial fluctuations in CNV amplitude reflect anticipatory adjustment of response caution. NeuroImage, 96:95-105, Aug. 2014. ISSN 10959572.

Frans A. Boiten. The effects of emotional behaviour on components of the respiratory cycle. Biological Psychology, 49(1-2):29-51, 1998. ISSN 03010511.

P. D. Bolls, A. Lang, and R. F. Potter. The Effects of Message Valence and Listener Arousal on Attention, Memory, and Facial Muscular Responses to Radio Advertisements. Communication Research, 28(5):627-651, Oct. 2001. ISSN 0093-6502.

Andrea Bonarini, Luca Mainardi, Matteo Matteucci, Simone Tognetti, and Roberto Colombo. Stress recognition in a robotic rehabilitation task. In Robotic Helpers: User Interaction, Interfaces and Companions in Assistive and Therapy Robotics, a Workshop at ACM/IEEE HRI, pages 41-48, 2008.

W. Boucsein. Electrodermal Activity. Springer Science \& Business Media, 2012.

Wolfram Boucsein, Don C. Fowles, Sverre Grimnes, Gershon Ben-Shakhar, Walton T. Roth, Michael E. Dawson, and Diane L. Filion. Publication recommendations for electrodermal measurements. Psychophysiology, 49 (8):1017-1034, Aug. 2012. ISSN 1540-5958.

Frédéric Bousefsaf, Choubeila Maaoui, and Alain Pruski. Remote detection of mental workload changes using cardiac parameters assessed with a low-cost webcam. Computers in Biology and Medicine, 53:154-163, 2014.

Margaret M Bradley. Emotion and motivation. In John T. Cacioppo, Louis G. Tassinary, and Gary G. Berntson, editors, Handbook of Psychophysiology, pages 602-642. Cambridge University Press, New York, NY, 2nd edition, 2000.

Nicolas Brodu, Fabien Lotte, and Anatole Lécuyer. Exploring two novel features for EEG-based brain-computer interfaces: Multifractal cumulants and predictive complexity. Neurocomputing, 79:87-94, Mar. 2012. ISSN 09252312. 
A. M. Brouwer, T. O. Zander, J. B. van Erp, J. E. Korteling, and A. W. Bronkhorst. Using neurophysiological signals that reflect cognitive or affective state: Six recommendations to avoid common pitfalls. Frontiers in Neuroscience, 9:136, 2015.

Anne-Marie Brouwer, Maarten A. Hogervorst, Jan B.F. van Erp, Tobias Heffelaar, Patrick H. Zimmerman, and Robert Oostenveld. Estimating workload using EEG spectral power and ERPs in the n-back task. Journal of Neural Engineering, 9(4):045008, 2012.

Jan M. Burg, Oliver T. Wolf, and Johannes Michalak. Mindfulness as self-regulated attention. Swiss Journal of Psychology, 71(3):135-139, 2012.

J. L. Hernández Cáceres, H. Foyaca Sibat, R. Hong, L. Garcia, M. Sautié, and V. Namugowa. Towards the estimation of the fractal dimension of heart rate variability data. Electron J. Biomed, 2(1), 2004.

John T. Cacioppo, Louis G. Tassinary, and Gary G. Berntson. Handbook of Psychophysiology. Cambridge University Press, 2000. ISBN 052162634X 9780521626347.

Rafael A. Calvo and Sidney D'Mello. Affect detection: An interdisciplinary review of models, methods, and their applications. IEEE Trans. Affective Comput., 1:18-37, 2010.

Rafael A. Calvo, Iain Brown, and Steve Scheding. Effect of experimental factors on the recognition of affective mental states through physiological measures. In LNCS AI 2009: Advances in Artificial Intelligence, volume 5866, pages 62-70. Springer, 2009.

Ryan T. Canolty and Robert T. Knight. The functional role of cross-frequency coupling. Trends in Cognitive Sciences, 14(11):506-515, Nov. 2010. ISSN 1364-6613.

N. Caramia, F. Lotte, and S. Ramat. Optimizing spatial filter pairs for EEG classification based on phase-synchronization. In 2014 IEEE International Conference on Acoustics, Speech and Signal Processing (ICASSP), pages 2049-2053, May 2014.

G. Chanel, C. Rebetez, M. Bétrancourt, and T. Pun. Emotion assessment from physiological signals for adaptation of game difficulty. IEEE Trans. Syst., Man, Cybern. A, 41(6):1052-1063, 2011.

Guillaume Chanel, Joep J. M. Kierkels, Mohammad Soleymani, and Thierry Pun. Short-term emotion assessment in a recall paradigm. International Journal of Human-Computer Studies, 67(8):607-627, 2009. 
Keng-hao Chang, Drew Fisher, John Canny, and Björn Hartmann. How's my mood and stress?: An efficient speech analysis library for unobtrusive monitoring on mobile phones. In Proceedings of the 6th International Conference on Body Area Networks, pages 71-77. ICST (Institute for Computer Sciences, Social-Informatics and Telecommunications Engineering), 2011.

Yongmin Chang and Sungmook Choi. Effects of seductive details evidenced by gaze duration. Neurobiology of learning and memory, 109:131-138, 2014.

Jui-Cheng Chen, Dorothea Hämmerer, Kevin D'Ostilio, Elias P. Casula, Louise Marshall, Chon-Haw Tsai, John C. Rothwell, and Mark J. Edwards. Bi-directional modulation of somatosensory mismatch negativity with transcranial direct current stimulation: An event related potential study. The Journal of Physiology, 592(Pt 4):745-757, Feb. 2014. ISSN 0022-3751.

Min Chen, Sergio Gonzalez, Athanasios Vasilakos, Huasong Cao, and Victor C. Leung. Body area networks: A survey. Mobile Networks and Applications, 16(2):171-193, 2011.

Mitch Cherniack, Hari Balakrishnan, Magdalena Balazinska, Donald Carney, Ugur Cetintemel, Ying Xing, and Stanley B. Zdonik. Scalable distributed stream processing. In CIDR, volume 3, pages 257-268, 2003.

Israel C. Christie and Bruce H. Friedman. Autonomic specificity of discrete emotion and dimensions of affective space: A multivariate approach. International Journal of Psychophysiology, 51(2):143-153, 2004.

Burcu Cinaz, Bert Arnrich, Roberto La Marca, and Gerhard Tröster. Monitoring of mental workload levels during an everyday life office-work scenario. Personal and Ubiquitous Computing, 17(2):229-239, 2013.

Gari D Clifford, Francisco Azuaje, and Patrick McSharry. Advanced Methods and Tools for ECG Data Analysis. Artech House, Inc., 2006.

Manfred Clynes. Sentography: Dynamic forms of communication of emotion and qualities. Computers in Biology and Medicine, 3(2):119-130, 1973.

Aitor Coca, Raymond J. Roberge, W. Jon Williams, Douglas P. Landsittel, Jeffrey B. Powell, and Andrew Palmiero. Physiological monitoring in firefighter ensembles: Wearable plethysmographic sensor vest versus standard equipment. Journal of Occupational and Environmental Hygiene, 7(2):109$114,2009$.

B. H. Cohen, R. J. Davidson, J. A. Senulis, C. D. Saron, and D. R. Weisman. Muscle tension patterns during auditory attention. Biological Psychology, 33(2-3):133-156, July 1992. ISSN 0301-0511.

Laura Lee Colgin. Mechanisms and Functions of Theta Rhythms. Annual Review of Neuroscience, 36(1):295-312, 2013. 
Cristina Conati and Heather Maclaren. Empirically building and evaluating a probabilistic model of user affect. User Model User-Adap Inter, 19(3): 267-303, 2009.

Isabelle Constant, Dominique Laude, Isabelle Murat, and Jean-Luc Elghozi. Pulse rate variability is not a surrogate for heart rate variability. Clinical Science, 97:391-397, 1999.

Ben Cowley and Niklas Ravaja. Learning in Balance: Using Oscillatory EEG Biomarkers of Attention, Motivation and Vigilance to Interpret GameBased Learning. Cogent Education, 1(1):1-23, 2014. ISSN 2331-186X.

Benjamin Cowley. Experimental Investigation of Saliency Processing in ADHD. 45th European Brain and Behaviour Society (EBBS) Meeting, 2013.

Benjamin Cowley. Psychophysiology and high-performance cognition - a brief review of the literature. PeerJ Preprints, 2015. ISSN 2167-9843.

Michael E Dawson, Anne M Schell, and Diane L Filion. The electrodermal system. In John T. Cacioppo, Louis G. Tassinary, and Gary G. Berntson, editors, Handbook of Psychophysiology. Cambridge University Press, Cambridge UK, 2nd edition, 2000.

Roelf Willem de Boer. Beat-to-beat blood pressure fluctuations and heart rate variability in man: Physiological relationships, analysis, techniques, and a simple model. PhD thesis, University of Amsterdam, 1985.

Tom A. de Graaf, Joachim Gross, Gavin Paterson, Tessa Rusch, Alexander T. Sack, and Gregor Thut. Alpha-Band Rhythms in Visual Task Performance: Phase-Locking by Rhythmic Sensory Stimulation. PLoS ONE, 8(3):e60035, Mar. 2013.

Maarten De Vos, Katharina Gandras, and Stefan Debener. Towards a truly mobile auditory brain-computer interface: Exploring the P300 to take away. International Journal of Psychophysiology: Official Journal of the International Organization of Psychophysiology, 91(1):46-53, Jan. 2014. ISSN 1872-7697.

Frédéric Dehais, Mickaël Causse, François Vachon, and Sébastien Tremblay. Cognitive conflict in human-automation interactions: A psychophysiological study. Applied Ergonomics, 43(3):588-595, 2012. ISSN 0003-6870.

Arnaud Delorme and Scott Makeig. EEGLAB: An open source toolbox for analysis of single-trial EEG dynamics including independent component analysis. Journal of Neuroscience Methods, 134(1):9-21, Mar. 2004. ISSN 0165-0270. 
Ahmet Cengizhan Dirican and Mehmet Göktürk. Psychophysiological measures of human cognitive states applied in human computer interaction. Procedia Computer Science, 3:1361-1367, 2011. ISSN 18770509.

Sidney D'Mello and Jacqueline Kory. Consistent but modest: A meta-analysis on unimodal and multimodal affect detection accuracies from 30 studies. In Consistent but modest: A meta-analysis on unimodal and multimodal affect detection accuracies from 30 studies, pages 31-38. ACM, 2012.

Sidney K. D'mello and Jacqueline Kory. A review and meta-analysis of multimodal affect detection systems. ACM Computing Surveys (CSUR), 47(3): 43, 2015 .

Andrew Duchowski. Eye tracking methodology: Theory and practice, volume 373. Springer Science \& Business Media, 2007.

Laura Dugué, Philippe Marque, and Rufin VanRullen. The Phase of Ongoing Oscillations Mediates the Causal Relation between Brain Excitation and Visual Perception. The Journal of Neuroscience, 31(33):11889-11893, Aug. 2011. ISSN 0270-6474, 1529-2401.

Thomas Eggert. Eye movement recordings: methods. Developments in Ophthalmology, 40:15-34, 2007. ISSN 0250-3751.

P. Ekman. Strong evidence for universals in facial expressions: A reply to Russell's mistaken critique. Psychological Bulletin, 115(2):268-287, Mar. 1994. ISSN 0033-2909.

Paul Ekman. Darwin, deception, and facial expression. Annals of the New York Academy of Sciences, 1000(1):205-221, 2003.

Paul Ekman and Wallace V. Friesen. Measuring facial movement. Environmental Psychology and Nonverbal Behavior, 1(1):56-75, 1976.

Paul Ed. Ekman and Richard J. Davidson. The Nature of Emotion: Fundamental Questions. Oxford University Press, 1994.

Moataz El Ayadi, Mohamed S. Kamel, and Fakhri Karray. Survey on speech emotion recognition: Features, classification schemes, and databases. Pattern Recognition, 44(3):572-587, 2011.

R. El Kaliouby and P. Robinson. Mind reading machines: Automated inference of cognitive mental states from video. In Systems, Man and Cybernetics, 2004 IEEE International Conference on, volume 1, pages 682-688, 2004.

Tolgay Ergenoglu, Tamer Demiralp, Zubeyir Bayraktaroglu, Mehmet Ergen, Huseyin Beydagi, and Yagiz Uresin. Alpha rhythm of the EEG modulates visual detection performance in humans. Cognitive Brain Research, 20(3): 376-383, Aug. 2004. ISSN 0926-6410. 
Justin R. Estepp and James C. Christensen. Physiological cognitive state assessment: Applications for designing effective human-machine systems. In Engineering in Medicine and Biology Society, EMBC, 2011 Annual International Conference of the IEEE, pages 6538-6541. IEEE, 2011.

Manuel J. A. Eugster, Tuukka Ruotsalo, Michiel M. Spapé, Ilkka Kosunen, Oswald Barral, Niklas Ravaja, Giulio Jacucci, and Samuel Kaski. Predicting term-relevance from brain signals. In Proceedings of the 37th International ACM SIGIR Conference on Research $\mathcal{E}$ Development in Information Retrieval, pages 425-434. ACM, 2014.

Florian Eyben, Martin Wöllmer, and Björn Schuller. Opensmile: The munich versatile and fast open-source audio feature extractor. In Proceedings of the International Conference on Multimedia, pages 1459-1462. ACM, 2010.

Stephen H. Fairclough. Fundamentals of physiological computing. Interact. Comput., 21(1-2):133-145, Jan. 2009a. ISSN 0953-5438.

Stephen H. Fairclough. Fundamentals of physiological computing. Interacting with Computers, 21(1):133-145, 2009b.

Stephen H. Fairclough and Kiel Gilleade. Advances in Physiological Computing. Springer, 2014.

Martino Fantato, Ben Cowley, and Niklas Ravaja. Arousing learning: A psychophysiological classifier for real-time emotion recognition in technology enhanced learning environments. In Paul Cunningham and Miriam Cunningham, editors, eChallenges e-2013, pages 1-8, Dublin, 2013. IIMC International Information Management Corporation.

B. Fowler. P300 as a measure of workload during a simulated aircraft landing task. Human Factors, 36(4):670-83, Dec. 1994. ISSN 0018-7208.

Alan J. Fridlund. Sociality of solitary smiling: Potentiation by an implicit audience. Journal of Personality and Social Psychology, 60(2):229-240, 1991.

Francois Xavier Gamelin, SERG Berthoin, Laurent Bosquet, et al. Validity of the polar s810 heart rate monitor to measure rr intervals at rest. Medicine and Science in Sports and Exercise, 38(5):887, 2006.

Liang Gao, Jinyang Liang, Chiye Li, and Lihong V Wang. Single-shot compressed ultrafast photography at one hundred billion frames per second. Nature, 516:74-77, 2014.

Anne Garde, Bjarne Laursen, Anker Jørgensen, and Bente Jensen. Effects of mental and physical demands on heart rate variability during computer work. European Journal of Applied Physiology, 87(4-5):456-461, 2002. 
Asif A. Ghazanfar and Drew Rendall. Evolution of human vocal production. Current Biology, 18(11):R457-R460, 2008.

Dimitris Giakoumis, Dimitrios Tzovaras, Konstantinos Moustakas, and George Hassapis. Automatic recognition of boredom in video games using novel biosignal moment-based features. IEEE Trans. Affective Comput., 2 (3):119-133, 2011.

E. Gil, M. Orini, R. Bailón, J. M. Vergara, L. Mainardi, and P. Laguna. Photoplethysmography pulse rate variability as a surrogate measurement of heart rate variability during non-stationary conditions. Physiological Measurement, 31(9):1271, 2010.

Keith W. Godin and John H. L. Hansen. Physical task stress and speaker variability in voice quality. EURASIP Journal on Audio, Speech, and Music Processing, 2015(1):1-13, 2015.

S. B. Gokturk, H. Yalcin, and C. Bamji. A time-of-flight depth sensor system description, issues and solutions. In Computer Vision and Pattern Recognition Workshop, 2004. CVPRW'04. Conference on, pages 35-35, June 2004.

Lukasz Golab and M. Tamer Özsu. Issues in data stream management. ACM Sigmod Record, 32(2):5-14, 2003.

Ary L. Goldberger, Luis A. N. Amaral, Leon Glass, Jeffrey M. Hausdorff, Plamen Ch. Ivanov, Roger G. Mark, Joseph E. Mietus, George B. Moody, Chung-Kang Peng, and H. Eugene Stanley. Physiobank, physiotoolkit, and physionet components of a new research resource for complex physiologic signals. Circulation, 101(23):e215-e220, 2000.

June Gruber, Douglas S. Mennin, Adam Fields, Amanda Purcell, and Greg Murray. Heart rate variability as a potential indicator of positive valence system disturbance: A proof of concept investigation. International Journal of Psychophysiology, 98(2):240-248, 2015.

Thomas Gruber and Matthias M. Müller. Oscillatory brain activity in the human EEG during indirect and direct memory tasks. Brain Research, 1097(1):194-204, June 2006. ISSN 0006-8993.

José Guerreiro, Raúl Martins, Hugo Silva, André Lourenço, and Ana L. N. Fred. Bitalino-a multimodal platform for physiological computing. In ICINCO (1), pages 500-506, 2013.

Hatice Gunes and Maja Pantic. Automatic, dimensional and continuous emotion recognition. International Journal of Synthetic Emotions (IJSE), 1(1): $68-99,2010$. 
Hatice Gunes and Björn Schuller. Categorical and dimensional affect analysis in continuous input: Current trends and future directions. Image and Vision Computing, 31(2):120-136, 2013.

Bahar Güntekin and Erol Başar. Gender differences influence brain's beta oscillatory responses in recognition of facial expressions. Neuroscience Letters, 424(2):94-99, Sep. 2007. ISSN 0304-3940.

Bahar Güntekin and Erol Başar. Event-related beta oscillations are affected by emotional eliciting stimuli. Neuroscience Letters, 483(3):173-178, Oct. 2010. ISSN 0304-3940.

Bahar Güntekin and Erol Başar. A review of brain oscillations in perception of faces and emotional pictures. Neuropsychologia, 58:33-51, May 2014. ISSN 0028-3932.

Thalia Harmony. The functional significance of delta oscillations in cognitive processing. Frontiers in Integrative Neuroscience, 7:83, 2013.

James D. Harnsberger, Harry Hollien, Camilo A. Martin, and Kevin A. Hollien. Stress and deception in speech: Evaluating layered voice analysis*. Journal of Forensic Sciences, 54(3):642-650, 2009.

Andrew Harver and Tyler S Lorig. Respiration. In J. T. Cacioppo, G. G. Bernston, and L. G. Tassinary, editors, Handbook of Psychophysiology, volume 2, chapter 10, pages 265-293. Cambridge University Press, New York, 2000.

Xiaochuan He, Rafik A. Goubran, and Xiaoping P. Liu. Evaluation of the correlation between blood pressure and pulse transit time. In Medical Measurements and Applications Proceedings (MeMeA), 2013 IEEE International Symposium on, pages 17-20. IEEE, 2013.

J. A. Healey and R. W. Picard. Detecting stress during real-world driving tasks using physiological sensors. IEEE Trans. Intell. Transport. Syst., 6 (2):156-166, 2005.

M. Heiden, E. Lyskov, M. Djupsjöbacka, F. Hellström, and A. G. Crenshaw. Effects of time pressure and precision demands during computer mouse work on muscle oxygenation and position sense. European Journal of Applied Physiology, 94(1-2):97-106, May 2005. ISSN 1439-6319.

Andreas Henelius and Jari Torniainen. MIDAS - Modular Integrated Distributed Analysis System. https://github.com/bwrc/midas/, 2015.

Andreas Henelius, Kati Hirvonen, Anu Holm, Jussi Korpela, and K. Muller. Mental workload classification using heart rate metrics. In Engineering in Medicine and Biology Society, 2009. EMBC 2009. Annual International Conference of the IEEE, pages 1836-1839. IEEE, 2009. 
P. Herman, G. Prasad, T. M. McGinnity, and D. Coyle. Comparative analysis of spectral approaches to feature extraction for EEG-based motor imagery classification. IEEE Transactions on Neural Systems and Rehabilitation Engineering, 16(4):317-326, Aug. 2008. ISSN 1534-4320.

Christoph S. Herrmann, Daniel Strüber, Randolph F. Helfrich, and Andreas K. Engel. EEG oscillations: From correlation to causality. International Journal of Psychophysiology, 2015. ISSN 0167-8760.

Eckhard H. Hess and James M. Polt. Pupil size in relation to mental activity during simple problem-solving. Science, 143(3611):1190-1192, 1964.

Ursula Hess, Rainer Banse, and Arvid Kappas. The intensity of facial expression is determined by underlying affective state and social situation. Journal of Personality and Social Psychology, 69(2):280-288, 1995.

Kati Hirvonen, Sampsa Puttonen, Kristian Gould, Jussi Korpela, Vilhelm F. Koefoed, and Kiti Müller. Improving the saccade peak velocity measurement for detecting fatigue. Journal of Neuroscience Methods, 187(2):199206, 2010 .

B. Hjorth. EEG analysis based on time domain properties. Electroencephalography and Clinical Neurophysiology, 29(3):306-310, 1970. ISSN 0013-4694.

Nis Hjortskov, Dag Rissén, Anne Katrine Blangsted, Nils Fallentin, Ulf Lundberg, and Karen Søgaard. The effect of mental stress on heart rate variability and blood pressure during computer work. European Journal of Applied Physiology, 92(1-2):84-89, 2004.

Maarten A. Hogervorst, Anne-Marie Brouwer, and Jan B. F. van Erp. Combining and comparing eeg, peripheral physiology and eye-related measures for the assessment of mental workload. Frontiers in Neuroscience, 8, 2014.

Ikuo Homma and Yuri Masaoka. Breathing rhythms and emotions. Experimental Physiology, 93(9):1011-1021, 2008. ISSN 1469-445X.

Jin-Hyuk Hong and Anind K. Dey. Affect modeling with field-based physiological responses. Interacting with Computers, page iwu014, 2014.

Clifford S. Hopkins, Roy J. Ratley, Daniel S. Benincasa, and John J. Grieco. Evaluation of voice stress analysis technology. In System Sciences, 2005. HICSS'05. Proceedings of the 38th Annual Hawaii International Conference on, pages 20b-20b. IEEE, 2005.

Jörn M. Horschig, Johanna M. Zumer, and Ali Bahramisharif. Hypothesisdriven methods to augment human cognition by optimizing cortical oscillations. Frontiers in Systems Neuroscience, 8:119, 2014. 
Heikki V. Huikuri, M. J Niemelä, Sirkku Ojala, Asko Rantala, M. J. Ikäheimo, and K. E. Airaksinen. Circadian rhythms of frequency domain measures of heart rate variability in healthy subjects and patients with coronary artery disease. effects of arousal and upright posture. Circulation, 90(1):121-126, 1994.

S. B. Hutton. Cognitive control of saccadic eye movements. Brain and Cognition, 68(3):327-340, 2008. ISSN 0278-2626. A Hundred Years of Eye Movement Research in Psychiatry.

Kazushi Hyoki, Masahiro Shigeta, Norifumi Tsuno, Yu Kawamuro, and Toshihiko Kinoshita. Quantitative electro-oculography and electroencephalography as indices of alertness. Electroencephalography and Clinical Neurophysiology, 106(3):213-219, 1998.

C. Iani, D. Gopher, A. J. Grunwald, and P. Lavie. Peripheral arterial tone as an on-line measure of load in a simulated flight task. Ergonomics, 50(7): 1026-1035, 2007.

Cristina Iani, Daniel Gopher, and Peretz Lavie. Effects of task difficulty and invested mental effort on peripheral vasoconstriction. Psychophysiology, 41 (5):789-798, 2004.

Michael Ingre, Torbjörn Åkerstedt, Björn Peters, Anna Anund, and Göran Kecklund. Subjective sleepiness, simulated driving performance and blink duration: Examining individual differences. Journal of Sleep Research, 15 (1):47-53, 2006.

Nikhil Iyengar, C. K. Peng, Raymond Morin, Ary L. Goldberger, and Lewis A. Lipsitz. Age-related alterations in the fractal scaling of cardiac interbeat interval dynamics. American Journal of Physiology-Regulatory, Integrative and Comparative Physiology, 271(4):R1078-R1084, 1996.

Robert JK Jacob. What you look at is what you get: Eye movement-based interaction techniques. In Proceedings of the SIGCHI Conference on Human factors in Computing Systems, pages 11-18. ACM, 1990.

Giulio Jacucci, Stephen Fairclough, and Erin T. Solovey. Physiological computing. Computer, 48(10):12-16, 2015.

Navendu Jain, Lisa Amini, Henrique Andrade, Richard King, Yoonho Park, Philippe Selo, and Chitra Venkatramani. Design, implementation, and evaluation of the linear road bnchmark on the stream processing core. In Proceedings of the 2006 ACM SIGMOD International Conference on Management of Data, pages 431-442. ACM, 2006. 
Marieke Jepma and Sander Nieuwenhuis. Pupil diameter predicts changes in the exploration-exploitation trade-off: Evidence for the adaptive gain theory. Journal of Cognitive Neuroscience, 23(7):1587-1596, 2011.

S. Jerritta, M. Murugappan, R. Nagarajan, and Khairunizam Wan. Physiological signals based human emotion recognition: A review. In Physiological Signals Based Human Emotion Recognition: A Review, pages 410-415. IEEE, 2011.

P. G. A. M. Jorna. Heart rate and workload variations in actual and simulated flight. Ergonomics, 36(9):1043-1054, 1993.

Yu. Kageyama, Masato Odagaki, and H. Hosaka. Wavelet analysis for quantification of mental stress stage by finger-tip photo-plethysmography. In Engineering in Medicine and Biology Society, 2007. EMBS 200\%. 29th Annual International Conference of the IEEE, pages 1846-1849. IEEE, 2007.

Arturas Kaklauskas, Edmundas Kazimieras Zavadskas, Marko Seniut, Gintautas Dzemyda, V. Stankevic, C. Simkevičius, T. Stankevic, R. Paliskiene, A. Matuliauskaite, and S. Kildiene. Web-based biometric computer mouse advisory system to analyze a user's emotions and work productivity. Engineering Applications of Artificial Intelligence, 24(6):928-945, 2011.

Sin-Hwa Kang, James H. Watt, and Sasi Kanth Ala. Social copresence in anonymous social interactions using a mobile video telephone. In Proceedings of the SIGCHI Conference on Human Factors in Computing Systems, CHI '08, pages 1535-1544, New York, NY, USA, 2008. ACM. ISBN 978-160558-011-1.

Romain E. Kania, Dana M. Hartl, Stéphane Hans, Shinji Maeda, Jacqueline Vaissiere, and Daniel F. Brasnu. Fundamental frequency histograms measured by electroglottography during speech: A pilot study for standardization. Journal of Voice, 20(1):18-24, 2006.

Ashish Kapoor, Winslow Burleson, and Rosalind W. Picard. Automatic prediction of frustration. International Journal of Human-Computer Studies, 65(8):724-736, 2007.

Emily S. Kappenman and Steven J. Luck. The Oxford Handbook of EventRelated Potential Components. Oxford University Press, Oxford, Dec. 2011. ISBN 9780195374148.

C. D. Katsis, G. Ganiatsas, and D. I. Fotiadis. An integrated telemedicine platform for the assessment of affective physiological states. Diagnostic Pathology, 1:16, 2006. 
C. D. Katsis, N. Katertsidis, G. Ganiatsas, and D. I. Fotiadis. Toward emotion recognition in car-racing drivers: A biosignal processing approach. IEEE Trans. Syst., Man, Cybern. A, 38(3):502-512, 2008.

Andreas Keil, Matthias M. Müller, Thomas Gruber, Christian Wienbruch, Margarita Stolarova, and Thomas Elbert. Effects of emotional arousal in the cerebral hemispheres: A study of oscillatory brain activity and eventrelated potentials. Clinical Neurophysiology, 112(11):2057-2068, Nov. 2001. ISSN 1388-2457.

Stéphanie Khalfa, Peretz Isabelle, Blondin Jean-Pierre, and Robert Manon. Event-related skin conductance responses to musical emotions in humans. Neuroscience Letters, 328(2):145-149, Aug. 2002. ISSN 0304-3940.

Sheraz Khan, Alexandre Gramfort, Nandita R. Shetty, Manfred G. Kitzbichler, Santosh Ganesan, Joseph M. Moran, Su Mei Lee, John D. E. Gabrieli, Helen B. Tager-Flusberg, Robert M. Joseph, Martha R. Herbert, Matti S. Hämäläinen, and Tal Kenet. Local and long-range functional connectivity is reduced in concert in autism spectrum disorders. Proceedings of the $\mathrm{Na}$ tional Academy of Sciences, 110(8):3107-3112, Feb. 2013. ISSN 0027-8424, 1091-6490.

Rami N Khushaba, Sarath Kodagoda, Sara Lal, and Gamini Dissanayake. Driver drowsiness classification using fuzzy wavelet-packet-based featureextraction algorithm. Biomedical Engineering, IEEE Transactions on, 58 (1):121-131, 2011.

Bjørg Elisabeth Kilavik, Manuel Zaepffel, Andrea Brovelli, William A. MacKay, and Alexa Riehle. The ups and downs of beta oscillations in sensorimotor cortex. Experimental Neurology, 245:15-26, July 2013. ISSN 0014-4886.

J. Kim and E. André. Emotion recognition based on physiological changes in music listening. IEEE Transactions on Pattern Analysis and Machine Intelligence, 30(12):2067-2083, 2008.

Jonghwa Kim. Bimodal Emotion Recognition using Speech and Physiological Changes. I-Tech Education and Publishing, Vienna, 2007.

K. H. Kim, S. W. Bang, and S. R. Kim. Emotion recognition system using short-term monitoring of physiological signals. Medical \& Biological Engineering \& Computing, 42(3):419-427, 2004.

Clemens Kirschbaum, K.-M. Pirke, and Dirk H. Hellhammer. The 'trier social stress test'-a tool for investigating psychobiological stress responses in a laboratory setting. Neuropsychobiology, 28(1-2):76-81, 1993. 
J. Matias Kivikangas, Guillaume Chanel, Ben Cowley, Inger Ekman, Mikko Salminen, Simo Järvelä, and Niklas Ravaja. A review on the use of psychophysiological methods in game research. Journal of Gaming and Virtual Worlds, 3(3):181-199, 2011.

Jaakko G. M. Klockars, Arja Hiller, Seppo Ranta, Pia Talja, Mark J. van Gils, and Tomi Taivainen. Spectral Entropy as a Measure of Hypnosis in Children. The Journal of the American Society of Anesthesiologists, 104 (4):708-717, Apr. 2006. ISSN 0003-3022.

Gennady G. Knyazev. EEG delta oscillations as a correlate of basic homeostatic and motivational processes. Neuroscience $\& 3$ Biobehavioral Reviews, 36(1):677-695, Jan. 2012. ISSN 0149-7634.

A. Koenig, D. Novak, X. Omlin, M. Pulfer, E. Perreault, L. Zimmerli, M. Mihelj, and R. Riener. Real-time closed-loop control of cognitive load in neurological patients during robot-assisted gait training. IEEE Transactions on Neural Systems and Rehabilitation Engineering: A Publication of the IEEE Engineering in Medicine and Biology Society, 19(4):453-464, 2011.

A. Kok. On the utility of P3 amplitude as a measure of processing capacity. Psychophysiology, 38(3):557-577, May 2001. ISSN 0048-5772.

V. Kolodyazhniy, S. D. Kreibig, J. J. Gross, W. T. Roth, and F. H. Wilhelm. An affective computing approach to physiological emotion specificity: Toward subject-independent and stimulus-independent classification of filminduced emotions. Psychophysiology, 48(7):908-922, 2011.

C. Kothe. Lab streaming layer (lsl), 2013.

Christian Andreas Kothe and Scott Makeig. BCILAB: A platform for braincomputer interface development. Journal of Neural Engineering, 10(5): 056014, 2013.

Arthur Kramer and John Spinks. Capacity views of human information processing. In J. Richard Jennings and Michael G. H. Coles, editors, Handbook of Cognitive Psychophysiology: Central and Autonomic Nervous System Approaches, pages 179-249. John Wiley \& Sons, Oxford, UK, 1991.

Arthur F. Kramer, Christopher D. Wickens, and Emanuel Donchin. An Analysis of the Processing Requirements of a Complex Perceptual-Motor Task. Human Factors: The Journal of the Human Factors and Ergonomics Society, 25(6):597-621, Dec. 1983.

S. D. Kreibig, F. H. Wilhelm, W. T. Roth, and J. J. Gross. Cardiovascular, electrodermal, and respiratory response patterns to fear- and sadnessinducing films. Psychophysiology, 44(5):787-806, 2007. 
Sylvia D. Kreibig. Autonomic nervous system activity in emotion: A review. Biological Psychology, 84(3):394-421, 2010.

Dean J. Krusienski, Dennis J. McFarland, and Jonathan R. Wolpaw. Value of amplitude, phase, and coherence features for a sensorimotor rhythmbased brain-computer interface. Brain Research Bulletin, 87(1):130-134, Jan. 2012. ISSN 0361-9230.

Brigitte M. Kudielka, Dirk H. Hellhammer, Clemens Kirschbaum, E. HarmonJones, and P. Winkielman. Ten years of research with the trier social stress test-revisited. Social Neuroscience: Integrating Biological and Psychological Explanations of Social Behavior, pages 56-83, 2007.

Kai Kuikkaniemi, Toni Laitinen, Marko Turpeinen, Timo Saari, Ilkka Kosunen, and Niklas Ravaja. The influence of implicit and explicit biofeedback in first-person shooter games. In Proceedings of the SIGCHI Conference on Human Factors in Computing Systems, CHI'10, pages 859-868, New York, NY, USA, 2010. ACM. ISBN 978-1-60558-929-9.

Martin Kusserow, Oliver Amft, and Gerhard Tröster. Monitoring stress arousal in the wild. IEEE Pervasive Computing, 12(2):28-37, 2013.

Helmut Karl Lackner, Nandu Goswami, Ilona Papousek, Andreas Roessler, Erik Konrad Grasser, Jean-Pierre Montani, Daniela Jezova, and Helmut Hinghofer-Szalkay. Time course of cardiovascular responses induced by mental and orthostatic challenges. International Journal of Psychophysiology, 75(1):48-53, 2010.

P. J. Lang, M. K. Greenwald, M. M. Bradley, and A. O. Hamm. Looking at pictures: Affective, facial, visceral, and behavioral reactions. Psychophysiology, 30(3):261-273, 1993.

Jeff T. Larsen, Catherine J. Norris, and John T. Cacioppo. Effects of positive and negative affect on electromyographic activity over zygomaticus major and corrugator supercilii. Psychophysiology, 40(5):776-85, Sep. 2003.

G. J. Lavigne, S. Khoury, S. Abe, T. Yamaguchi, and K. Raphael. Bruxism physiology and pathology: An overview for clinicians*. Journal of Oral Rehabilitation, 35(7):476-494, 2008.

C. K. Lee, H. S. Shin, S. D. Min, Y. H. Yun, and M. H. Lee. A study on comparison PPG variability with heart rate variability in the sitting position during paced respiration. In World Congress on Medical Physics and Biomedical Engineering, September 7-12, 2009, Munich, Germany, pages 1703-1705. Springer, 2010. 
Jaehoon Lee, Min Hong, and Sungyong Ryu. Sleep monitoring system using kinect sensor. International Journal of Distributed Sensor Networks, 2015, 2015.

Enrique Leon, Graham Clarke, Victor Callaghan, and Francisco Sepulveda. A user-independent real-time emotion recognition system for software agents in domestic environments. Engineering Applications of Artificial Intelligence, 20(3):337-345, 2007.

Florent Levillain, Joseph Onderi Orero, Maria Rifqi, and Bernadettele Bouchon-Meunier. Characterizing player's experience from physiological signals using fuzzy decision trees. In Computational Intelligence and Games (CIG), 2010 IEEE Symposium on, pages 75-82. IEEE, 2010.

René Lien, Melanie Neijts, Gonneke Willemsen, and Eco J. C. de Geus. Ambulatory measurement of the ECG t-wave amplitude. Psychophysiology, 52 (2):225-237, 2015.

Eunho Lim, Hyo-Ki Lee, Hyoun-Seok Myoung, and Kyoung-Joung Lee. Development of a noncontact heart rate monitoring system for sedentary behavior based on an accelerometer attached to a chair. Physiological Measurement, 36(3):N61, 2015.

James C. Lin, Joseph Kiernicki, Martin Kiernicki, and Paul B Wollschlaeger. Microwave apexcardiography. Microwave Theory and Techniques, IEEE Transactions on, 27(6):618-620, 1979.

Wan-Hua Lin, Dan Wu, Chunyue Li, Heye Zhang, and Yuan-Ting Zhang. Comparison of heart rate variability from PPG with that from ECG. In The International Conference on Health Informatics, pages 213-215. Springer, 2014.

C. Lisetti, F. Nasoz, C. LeRouge, O. Ozyer, and K. Alvarez. Developing multimodal intelligent affective interfaces for tele-home health care. International Journal of Human-Computer Studies, 59(1-2):245-255, 2003.

Christine L. Lisetti and Fatma Nasoz. Using noninvasive wearable computers to recognize human emotions from physiological signals. EURASIP Journal on Applied Signal Processing, 2004:1672-1687, 2004.

J. E. Lisman and M. A. Idiart. Storage of $7+/-2$ short-term memories in oscillatory subcycles. Science (New York, N.Y.), 267(5203):1512-1515, Mar. 1995. ISSN 0036-8075.

C. Liu, K. Conn, N. Sarkar, and W. Stone. Online affect detection and robot behavior adaptation for intervention of children with autism. IEEE Trans. Robot., 24(4):883-896, 2008. 
Changchun Liu, Pramila Agrawal, Nilanjan Sarkar, and Shuo Chen. Dynamic difficulty adjustment in computer games through real-time anxiety-based affective feedback. International Journal of Human-Computer Interaction, 25(6):506-529, 2009.

Y. Liu, J. M. Ritchie, T. Lim, Z. Kosmadoudi, A. Sivanathan, and R. C. W. Sung. A fuzzy psycho-physiological approach to enable the understanding of an engineer's affect status during CAD activities. Computer-Aided Design, 2013.

Scott E. Lively, David B. Pisoni, W. Van Summers, and Robert H. Bernacki. Effects of cognitive workload on speech production: Acoustic analyses and perceptual consequences. The Journal of the Acoustical Society of America, 93(5):2962-2973, 1993.

Irene Lopatovska and Ioannis Arapakis. Theories, methods and current research on emotions in library and information science, information retrieval and human-computer interaction. Information Processing $\& 3$ Management, 47(4):575-592, 2011.

Fernando Lopes da Silva. The rhythmic slow activity (theta) of the limbic cortex: an oscillation in search of a function. In Erol Başar and Theodore H. Bullock, editors, Induced Rhythms in the Brain, Brain Dynamics, pages 83102. Birkhäuser Boston, 1992. ISBN 978-1-4757-1283-4 978-1-4757-1281-0.

Fernando Lopes da Silva. EEG and MEG: Relevance to neuroscience. Neuron, 80(5):1112-1128, Dec. 2013. ISSN 0896-6273.

F. Lotte. A new feature and associated optimal spatial filter for EEG signal classification: Waveform Length. In 2012 21st International Conference on Pattern Recognition (ICPR), pages 1302-1305, Nov. 2012.

Fabien Lotte. A tutorial on EEG signal-processing techniques for mental-state recognition in Brain-Computer Interfaces. In Eduardo Reck Miranda and Julien Castet, editors, Guide to Brain-Computer Music Interfacing, pages 133-161. Springer London, 2014. ISBN 978-1-4471-6583-5 978-1-4471-65842.

G. Lu, F. Yang, J. A. Taylor, and J. F. Stein. A comparison of photoplethysmography and ECG recording to analyse heart rate variability in healthy subjects. Journal of Medical Engineering 83 Technology, 33(8):634-641, 2009.

Steven J. Luck. An Introduction to the Event-Related Potential Technique. MIT Press, Cambridge MA, 2nd edition, 2014. ISBN 9780262525855. 
Kristian Lukander, Sharman Jagadeesan, Huageng Chi, and Kiti Müller. Omg!: A new robust, wearable and affordable open source mobile gaze tracker. In Proceedings of the 15th International Conference on HumanComputer Interaction with Mobile Devices and Services, pages 408-411. ACM, 2013.

M. Lyons, S. Akamatsu, M. Kamachi, and J. Gyoba. Coding facial expressions with Gabor wavelets. In Proceedings Third IEEE International Conference on Automatic Face and Gesture Recognition, pages 200-205, Nara, Japan, 1998. IEEE Comput. Soc. ISBN 0-8186-8344-9.

Lili Ma, Tianmin Xu, and Jiuxiang Lin. Validation of a three-dimensional facial scanning system based on structured light techniques. Computer Methods and Programs in Biomedicine, 94(3):290-298, 2009. ISSN 01692607.

T. Ma and Y. T. Zhang. A correlation study on the variabilities in pulse transit time, blood pressure, and heart rate recorded simultaneously from healthy subjects. In Engineering in Medicine and Biology Society, 2005. IEEEEMBS 2005. 27th Annual International Conference of the, pages 996-999. IEEE, 2005.

Ludo Maat and Maja Pantic. Gaze-X: Adaptive, Affective, Multimodal Interface for Single-User Office Scenarios, pages 251-271. Springer, Berlin, Heidelberg, 2007. ISBN 978-3-540-72348-6.

K. Madden and G. K. Savard. Effects of mental state on heart rate and blood pressure variability in men and women. Clinical Physiology, 15(6):557-569, 1995.

Marek Malik, J. Thomas Bigger, A. John Camm, Robert E. Kleiger, Alberto Malliani, Arthur J. Moss, and Peter J. Schwartz. Heart rate variability standards of measurement, physiological interpretation, and clinical use. European Heart Journal, 17(3):354-381, 1996.

Jaakko Malmivuo and Robert Plonsey. Bioelectromagnetism: Principles and Applications of Bioelectric and Biomagnetic Fields. Oxford University Press, 1995.

Lucas Malta, Chiyomi Miyajima, Norihide Kitaoka, and Kazuya Takeda. Analysis of real-world driver's frustration. Intelligent Transportation Systems, IEEE Transactions on, 12(1):109-118, 2011.

Regan L. Mandryk and M. Stella Atkins. A fuzzy physiological approach for continuously modeling emotion during interaction with play technologies. International Journal of Human-Computer Studies, 65(4):329-347, 2007. 
Sandra P. Marshall. Identifying cognitive state from eye metrics. Aviation, Space, and Environmental Medicine, 78(Supplement 1):B165-B175, 2007.

S. P. Marshall. The index of cognitive ativity: Measuring cognitive workload. In Human Factors and Power Plants, 2002. Proceedings of the 2002 IEEE 7th Conference on, pages 7-5-7-9, 2002.

Brais Martinez and Michel F. Valstar. Advances, challenges, and opportunities in automatic facial expression recognition. In Advances in Face Detection and Facial Image Analysis, pages 63-100. Springer, 2016.

Kyle E. Mathewson, Chandramallika Basak, Edward L. Maclin, Kathy A. Low, Walter R. Boot, Arthur F. Kramer, Monica Fabiani, and Gabriele Gratton. Different slopes for different folks: Alpha and delta EEG power predict subsequent video game learning rate and improvements in cognitive control tasks. Psychophysiology, 49(12):1558-1570, Dec. 2012. ISSN 14698986.

S. E. Mathiassen, J. Winkel, and G. M. Hägg. Normalization of surface EMG amplitude from the upper trapezius muscle in ergonomic studies-a review. Journal of Electromyography and Kinesiology, 5(4):197-226, 1995.

Albert Mehrabian. Comparison of the pad and panas as models for describing emotions and for differentiating anxiety from depression. Journal of Psychopathology and Behavioral Assessment, 19(4):331-357, 1997.

Bjorn Merker. Cortical gamma oscillations: The functional key is activation, not cognition. Neuroscience \& Biobehavioral Reviews, 37(3):401-417, Mar. 2013. ISSN 0149-7634.

Susann Meyberg, Markus Werkle-Bergner, Werner Sommer, and Olaf Dimigen. Microsaccade-related brain potentials signal the focus of visuospatial attention. NeuroImage, 104:79-88, 2015. ISSN 1053-8119.

Shinji Miyake, Shimpei Yamada, Takuro Shoji, Yasuhiko Takae, Nobuyuki Kuge, and Tomohiro Yamamura. Physiological responses to workload change. A test/retest examination. Applied Ergonomics, 40(6):987-996, 2009.

Yasser Mohammad and Toyoaki Nishida. Using physiological signals to detect natural interactive behavior. Appl. Intell., 33(1):79-92, 2010.

Neema Moraveji, Ben Olson, Truc Nguyen, Mahmoud Saadat, Yaser Khalighi, Roy Pea, and Jeffrey Heer. Peripheral paced respiration: Influencing user physiology during information work. In Proceedings of the 24th Annual ACM Symposium on User Interface Software and Technology, UIST '11, pages 423-428, New York, NY, USA, 2011. ACM. ISBN 978-1-4503-07161. 
Masahiro Mori, Karl F. MacDorman, and Norri Kageki. The uncanny valley [from the field]. Robotics \& Automation Magazine, IEEE, 19(2):98-100, 2012.

T. L. Morris and James C. Miller. Electrooculographic and performance indices of fatigue during simulated flight. Biological Psychology, 42(3):343360, 1996.

Christian Mühl, Camille Jeunet, and Fabien Lotte. EEG-based workload estimation across affective contexts. Frontiers in Neuroscience, 8, 2014.

Ben Mulder, Hans Veldman, Freddy van der Veen, Arie van Roon, Heinz Rüddel, Hartmut Schächinger, and Bert Mulder. On the effects of mental task performance on heart rate, blood pressure and its variability measures. Blood Pressure and Heart Rate Variability: Computer Analysis, Modelling and Clinical Applications, 4:153, 1993.

Lambertus Johannes Maria Mulder. Assessment of cardiovascular reactivity by means of spectral analysis. Biological Psychology, 1988.

L. J. Mulder, J. B. Veldman, H. Rüddel, H. W. Robbe, and G. Mulder. On the usefulness of finger blood-pressure measurements for studies on mental workload. Homeostasis in Health and Disease: International Journal Devoted to Integrative Brain Functions and Homeostatic Systems, 33(1-2): 47-60, 1990.

M. Myrtek, E. Deutschmann-Janicke, H. Strohmaier, W. Zimmermann, S. Lawerenz, G. Brügner, and W. Müller. Physical, mental, emotional, and subjective workload components in train drivers. Ergonomics, 37(7): 1195-1203, 1994.

Lennart Nacke and Craig A. Lindley. Flow and immersion in first-person shooters: Measuring the player's gameplay experience. In Proceedings of the 2008 Conference on Future Play: Research, Play, Share, pages 81-88. ACM, 2008.

Yoshio Nakamura, Yoshiharu Yamamoto, and I. Muraoka. Autonomic control of heart rate during physical exercise and fractal dimension of heart rate variability. Journal of Applied Physiology, 74:875-875, 1993.

Fatma Nasoz, Kaye Alvarez, Christine L. Lisetti, and Neal Finkelstein. Emotion recognition from physiological signals using wireless sensors for presence technologies. Cognition, Technology \& Work, 6(1):4-14, 2004.

Fatma Nasoz, Christine L. Lisetti, and Athanasios V. Vasilakos. Affectively intelligent and adaptive car interfaces. Information Sciences, 180(20):3817$3836,2010$. 
S. V. Nesterov, V. P. Nesterov, and A. I. Burdygin. The effect of respiratory frequency on heart rate variability. Doklady Biological Sciences, 400(1): $25-27,2005$.

Leonardo Neumeyer, Bruce Robbins, Anish Nair, and Anand Kesari. S4: Distributed stream computing platform. In Data Mining Workshops (ICDMW), 2010 IEEE International Conference on, pages 170-177. IEEE, 2010.

Peter Nickel and Friedhelm Nachreiner. Sensitivity and diagnosticity of the 0.1-hz component of heart rate variability as an indicator of mental workload. Human Factors: The Journal of the Human Factors and Ergonomics Society, 45(4):575-590, 2003.

Hiroshi Nittono, Aya Hamada, and Tadao Hori. Brain Potentials after Clicking a Mouse: A New Psychophysiological Approach to Human-Computer Interaction. Human Factors: The Journal of the Human Factors and Ergonomics Society, 45(4):591-599, Jan. 2003. ISSN 1547-8181.

MaryAnn P. Noonan, Nika Adamian, Alexandra Pike, Frida Printzlau, Ben M. Crittenden, and Mark G. Stokes. Distinct Mechanisms for Distractor Suppression and Target Facilitation. The Journal of Neuroscience, 36(6):17971807, Feb. 2016. ISSN 0270-6474, 1529-2401.

D. Novak, M. Mihelj, J. Ziherl, A. Olenšek, and M. Munih. Psychophysiological measurements in a biocooperative feedback loop for upper extremity rehabilitation. IEEE Transactions on Neural Systems and Rehabilitation Engineering: A Publication of the IEEE Engineering in Medicine and Biology Society, 19(4):400-410, 2011.

Domen Novak, Matjaž Mihelj, and Marko Munih. A survey of methods for data fusion and system adaptation using autonomic nervous system responses in physiological computing. Interacting with Computers, 24(3): 154-172, 2012.

Redmond G. O'Connell, Mark A. Bellgrove, Paul M. Dockree, Adam Lau, Michael Fitzgerald, and Ian H. Robertson. Self-Alert Training: Volitional modulation of autonomic arousal improves sustained attention. Neuropsychologia, 46(5):1379-1390, Apr. 2008. ISSN 0028-3932.

Redmond G. O'Connell, Paul M. Dockree, and Simon P. Kelly. A supramodal accumulation-to-bound signal that determines perceptual decisions in humans. Nature Neuroscience, 15(12):1729-1735, Dec. 2012. ISSN 1546-1726. 
Flavio T. P. Oliveira, Anne Aula, and Daniel M. Russell. Discriminating the relevance of web search results with measures of pupil size. In Proceedings of the SIGCHI Conference on Human Factors in Computing Systems, CHI '09, pages 2209-2212, New York, NY, USA, 2009. ACM. ISBN 978-1-60558246-7.

Oskar Palinko and Andrew L. Kun. Exploring the influence of light and cognitive load on pupil diameter in driving simulator studies. In Proceedings of the Sixth International Driving Symposium on Human Factors in Driver Assessment, Training and Vehicle Design, pages 329-336. Public Policy Center, University of Iowa Iowa City, 2011.

Oskar Palinko and Andrew L. Kun. Exploring the effects of visual cognitive load and illumination on pupil diameter in driving simulators. In Proceedings of the Symposium on Eye Tracking Research and Applications, ETRA '12, pages 413-416, New York, NY, USA, 2012. ACM. ISBN 978-1-4503$1221-9$.

J. Matias Palva, Satu Palva, and Kai Kaila. Phase Synchrony among Neuronal Oscillations in the Human Cortex. The Journal of Neuroscience, 25(15): 3962-3972, Apr. 2005. ISSN 0270-6474, 1529-2401.

A. Pantelopoulos and N. G. Bourbakis. A Survey on Wearable Sensor-Based Systems for Health Monitoring and Prognosis. IEEE Transactions on Systems, Man, and Cybernetics, Part C (Applications and Reviews), 40(1): 1-12, Jan. 2010. ISSN 1094-6977.

Christos Papadelis, Zhe Chen, Chrysoula Kourtidou-Papadeli, Panagiotis D. Bamidis, Ioanna Chouvarda, Evangelos Bekiaris, and Nikos Maglaveras. Monitoring sleepiness with on-board electrophysiological recordings for preventing sleep-deprived traffic accidents. Clinical Neurophysiology, 118(9): 1906-1922, 2007.

Gianfranco Parati, J. Philip Saul, Marco Di Rienzo, and Giuseppe Mancia. Spectral analysis of blood pressure and heart rate variability in evaluating cardiovascular regulation a critical appraisal. Hypertension, 25(6):12761286, 1995.

Byungho Park. Psychophysiology as a Tool for HCI Research: Promises and Pitfalls. In Julie A. Jacko, editor, Human-Computer Interaction. New Trends: 13th International Conference, HCI International, July 19-24, volume 5610 of Lecture Notes in Computer Science, pages 141-148, San Diego, CA, USA, 2009. Springer, Berlin, Heidelberg. ISBN 978-3-642-02573-0. 
Timo Partala and Veikko Surakka. Pupil size variation as an indication of affective processing. International Journal of Human-Computer Studies, 59 (1-2):185-198, 2003. ISSN 1071-5819. Applications of Affective Computing in Human-Computer Interaction.

Ioannis Pavlidis, Jonathan Dowdall, Nanfei Sun, Colin Puri, Jin Fei, and Marc Garbey. Interacting with human physiology. Computer Vision and Image Understanding, 108(1):150-170, 2007.

J. Peñáz. Photoelectric measurement of blood pressure, volume and flow in the finger. In Digest of the 10th International Conference on Medical and Biological Engineering, volume 104. International Federation for Medical and Biological Engineering, Publishers New York, 1973.

Marco Pedrotti, Mohammad Ali Mirzaei, Adrien Tedesco, Jean-Rémy Chardonnet, Frédéric Mérienne, Simone Benedetto, and Thierry Baccino. Automatic stress classification with pupil diameter analysis. International Journal of Human-Computer Interaction, 30(3):220-236, 2014.

Christian Peter and Antje Herbon. Emotion representation and physiology assignments in digital systems. Interacting with Computers, 18(2):139-170, 2006.

G. Pfurtscheller and F. H. Lopes da Silva. Event-related EEG/MEG synchronization and desynchronization: Basic principles. Clinical Neurophysiology, 110(11):1842-1857, Nov. 1999. ISSN 1388-2457.

R. W. Picard, S. Fedor, and Y. Ayzenberg. Multiple Arousal Theory and Daily-Life Electrodermal Activity Asymmetry. Emotion Review, 8(1):6275, Mar. 2015. ISSN 1754-0739.

Rosalind W. Picard, Elias Vyzas, and Jennifer Healey. Toward machine emotional intelligence: Analysis of affective physiological state. Pattern Analysis and Machine Intelligence, IEEE Transactions on, 23(10):1175-1191, 2001.

T. W. Picton. The P300 wave of the human event-related potential. Journal of Clinical Neurophysiology: Official Publication of the American Electroencephalographic Society, 9(4):456-479, 1992. ISSN 0736-0258.

Kurt Plarre, Andrew Raij, Syed Monowar Hossain, Amin Ahsan Ali, Motohiro Nakajima, Mustafa al'Absi, Emre Ertin, Thomas Kamarck, Santosh Kumar, and Marcia Scott. Continuous inference of psychological stress from sensory measurements collected in the natural environment. In Information Processing in Sensor Networks (IPSN), 2011 10th International Conference on, pages 97-108. IEEE, 2011. 
Ming-Zher Poh, Daniel J. McDuff, and Rosalind W. Picard. Non-contact, automated cardiac pulse measurements using video imaging and blind source separation. Optics Express, 18(10):10762-10774, 2010.

H. Poikonen, V. Alluri, E. Brattico, O. Lartillot, M. Tervaniemi, and M. Huotilainen. Event-related brain responses while listening to entire pieces of music. Neuroscience, 312:58-73, Nov. 2016. ISSN 1873-7544.

Jonathan Posner, James A. Russell, and Bradley S. Peterson. The circumplex model of affect: An integrative approach to affective neuroscience, cognitive development, and psychopathology. Development and Psychopathology, 17 (03):715-734, 2005.

P. Rainville, A. Bechara, N. Naqvi, and A. R. Damasio. Basic emotions are associated with distinct patterns of cardiorespiratory activity. Int. J. Psychophysiol, 61(1):5-18, 2006.

Pramila Rani, Changchun Liu, Nilanjan Sarkar, and Eric Vanman. An empirical study of machine learning techniques for affect recognition in humanrobot interaction. Pattern Anal. Applic., 9(1):58-69, 2006.

N. Ravaja, G. Bente, J. Kätsyri, M. Salminen, and T. Takala. Virtual character facial expressions influence human brain and facial EMG activity in a decision-making game. In IEEE Transactions on Affective Computing (submitted), 2015.

Niklas Ravaja. Contributions of Psychophysiology to Media Research: Review and Recommendations. Media Psychology, 6(2):193-235, 2004.

Niklas Ravaja, Timo Saari, Kari Kallinen, and Jari Laarni. The Role of Mood in the Processing of Media Messages From a Small Screen: Effects on Subjective and Physiological Responses. Media Psychology, 8(3):239265, Aug. 2006a. ISSN 1521-3269.

Niklas Ravaja, Timo Saari, Marko Turpeinen, Jari Laarni, Mikko Salminen, and Matias Kivikangas. Spatial Presence and Emotions during Video Game Playing: Does It Matter with Whom You Play? Presence: Teleoperators and Virtual Environments, 15(4):381-392, 2006b.

W. J. Ray and H. W. Cole. EEG alpha activity reflects attentional demands, and beta activity reflects emotional and cognitive processes. Science, 228 (4700):750-752, May 1985. ISSN 0036-8075, 1095-9203.

Keith Rayner. Eye movements and attention in reading, scene perception, and visual search. The Quarterly Journal of Experimental Psychology, 62 (8):1457-1506, 2009. 
Susan A Reedijk, Anne Bolders, Lorenza S Colzato, and Bernhard Hommel. Eliminating the attentional blink through binaural beats: A case for tailored cognitive enhancement. Frontiers in Psychiatry, 6:82, Jan. 2015. ISSN 1664-0640.

Lingyun Ren, Yun Seo Koo, Yazhou Wang, and Aly E. Fathy. Noncontact heartbeat detection using UWB impulse doppler radar. In 2015 IEEE Topical Conference on Biomedical Wireless Technologies, Networks, and Sensing Systems (BioWireleSS), pages 1-3. IEEE, Jan. 2015. ISBN 978-14799-5511-4.

Yann Renard, Fabien Lotte, Guillaume Gibert, Marco Congedo, Emmanuel Maby, Vincent Delannoy, Olivier Bertrand, and Anatole Lécuyer. OpenViBE: An open-source software platform to design, test, and use braincomputer interfaces in real and virtual environments. Presence: Teleoperators and Virtual Environments, 19(1):35-53, 2010.

G. Rigas, C. D. Katsis, G. Ganiatsas, and D. I. Fotiadis. A User Independent, Biosignal Based, Emotion Recognition Method, pages 314-318. Springer, Berlin, Heidelberg, 2007. ISBN 978-3-540-73078-1.

George Rigas, Yorgos Goletsis, Panagiota Bougia, and Dimitrios I. Fotiadis. Towards driver's state recognition on real driving conditions. International Journal of Vehicular Technology, 2011:1-14, 2011.

Christopher Ring, Victoria E Burns, and Douglas Carroll. Shifting hemodynamics of blood pressure control during prolonged mental stress. Psychophysiology, 39(5):585-590, 2002.

H. W. Robbe, L. J. Mulder, H. Rüddel, Wolf A. Langewitz, J. B. Veldman, and Gusbertus Mulder. Assessment of baroreceptor reflex sensitivity by means of spectral analysis. Hypertension, 10(5):538-543, 1987.

A. H. Roscoe. Heart rate as a psychophysiological measure for in-flight workload assessment. Ergonomics, 1993.

Alan H. Roscoe. Assessing pilot workload. why measure heart rate, hrv and respiration? Biological Psychology, 34(2):259-287, 1992.

Constantin A. Rothkopf, Dana H. Ballard, and Mary M. Hayhoe. Task and context determine where you look. Journal of Vision, 7(14):16, 2015.

James A. Russell. A circumplex model of affect. Journal of Personality and Social Psychology, 39(6):1161-1178, 1980.

George H. Sage and Bonnie Bennett. The Effects of Induced Arousal on Learning and Performance of a Pursuit Motor Skill. Research Quarterly. American Association for Health, Physical Education and Recreation, 44 (2):140-149, 1973. ISSN 1067-1188. 
Takuya Sakamoto, Ryohei Imasaka, Hirofumi Taki, Toru Sato, Mototaka Yoshioka, Kenichi Inoue, Takeshi Fukuda, and Hiroyuki Sakai. Accurate heartbeat monitoring using ultra-wideband radar. IEICE Electronics Express, 12(3):20141197-20141197, Feb. 2015. ISSN 1349-2543.

George E. Sakr, Imad H. Elhajj, and Huda Abou-Saad Huijer. Support vector machines to define and detect agitation transition. Affective Computing, IEEE Transactions on, 1(2):98-108, 2010.

Mikko Salminen, Niklas Ravaja, Kari Kallinen, and Timo Saari. Mediated Cues of Group Emotion during Knowledge-Work Tasks: Effects on Subjective and Physiological Responses. Interact. Comput., 25(1):60-73, Jan. 2013.

Ashok Samal and Prasana A. Iyengar. Automatic recognition and analysis of human faces and facial expressions: A survey. Pattern Recognition, 25(1): 65-77, 1992.

B. Mca. Savers, H. A. Beagley, and W. R. Henshall. The mechanism of auditory evoked EEG responses. Nature, 247(5441):481-483, Feb. 1974. ISSN 0028-0836.

Gerwin Schalk, Dennis J. McFarland, Thilo Hinterberger, Niels Birbaumer, and Jonathan R. Wolpaw. BCI2000: A general-purpose brain-computer interface (BCI) system. Biomedical Engineering, IEEE Transactions on, 51(6):1034-1043, 2004.

Klaus R. Scherer. Vocal communication of emotion: A review of research paradigms. Speech Communication, 40(1):227-256, 2003.

Moritz Schiff, R. Guichard de Choisity, and Jean-Baptiste Baillière. La pupille considérée comme esthésiomètre. J.-B. Baillière et fils, 1875.

B. Schuller, S. Steidl, and Batliner A. Repository of the interspeech computational paralinguistics challenge (compare) series. http://www. compare. openaudio.eu/, 2015. Accessed:2016-01-15.

Björn Schuller, Ronald Müller, Florian Eyben, Jürgen Gast, Benedikt Hörnler, Martin Wöllmer, Gerhard Rigoll, Anja Höthker, and Hitoshi Konosu. Being bored? recognising natural interest by extensive audiovisual integration for real-life application. Image and Vision Computing, 27(12):1760-1774, 2009.

C. Scully, Jinseok Lee, J. Meyer, A. M. Gorbach, D. Granquist-Fraser, Y. Mendelson, and K. H. Chon. Physiological parameter monitoring from optical recordings with a mobile phone. Biomedical Engineering, IEEE Transactions on, 59(2):303-306, Feb. 2012. ISSN 0018-9294. 
Nandakumar Selvaraj, Ashok Jaryal, Jayashree Santhosh, Kishore K. Deepak, and Sneh Anand. Assessment of heart rate variability derived from fingertip photoplethysmography as compared to electrocardiography. Journal of Medical Engineering \&3 Technology, 32(6):479-484, 2008.

C. Setz, B. Arnrich, J. Schumm, R. La Marca, G. Troster, and U. Ehlert. Discriminating stress from cognitive load using a wearable EDA device. IEEE Transactions on Information Technology in Biomedicine, 14(2):410417, Mar. 2010a. ISSN 1089-7771.

C. Setz, B. Arnrich, J. Schumm, R. La Marca, G. Tröster, and U. Ehlert. Discriminating stress from cognitive load using a wearable EDA device. IEEE transactions on information technology in biomedicine: A publication of the IEEE Engineering in Medicine and Biology Society, 14(2):410-417, 2010b.

Cornelia Setz, Johannes Schumm, Claudia Lorenz, Bert Arnrich, and Gerhard Tröster. Combining worthless sensor data. In Measuring Mobile Emotions Workshop at MobileHCI, 2009.

Caifeng Shan, Shaogang Gong, and Peter W. McOwan. Facial expression recognition based on Local Binary Patterns: A comprehensive study. Image and Vision Computing, 27(6):803-816, 2009. ISSN 0262-8856.

Nandita Sharma and Tom Gedeon. Modeling observer stress for typical real environments. Expert Systems with Applications, 41(5):2231-2238, 2014.

L. Shen, M. Wang, and R. Shen. Affective e-learning: Using "emotional" data to improve learning in pervasive learning environment. Journal of Educational Technology \&3 Society, 12(2):176-189, 2009.

Hugo Plácido Da Silva, Stephen Fairclough, Andreas Holzinger, Robert Jacob, and Desney Tan. Introduction to the special issue on physiological computing for human-computer interaction. ACM Transactions on ComputerHuman Interaction (TOCHI), 21(6):29, 2015.

R. F. Simons, B. H. Detenber, T. M. Roedema, and J. E. Reiss. Emotion processing in three systems: The medium and the message. Psychophysiology, 36(5):619-27, Sep. 1999. ISSN 0048-5772.

E. J. Sirevaag, A. F. Kramer, C. D. Wickens, M. Reisweber, D. L. Strayer, and J. F. Grenell. Assessment of pilot performance and mental workload in rotary wing aircraft. Ergonomics, 36(9):1121-40, Sep. 1993. ISSN 00140139 . 
Michiel M. Spapé, J. Matias Kivikangas, Simo Järvelä, Ilkka Kosunen, Giulio Jacucci, and Niklas Ravaja. Keep your opponents close: Social context affects eeg and femg linkage in a turn-based computer game. PloS ONE, 8 (11):e78795, 2013.

Michiel M Spapé, Eve E. Hoggan, Giulio Jacucci, and Niklas Ravaja. The meaning of the virtual Midas touch: An ERP study in economic decision making. Psychophysiology, 52(3):378-387, 2015.

Quentin Stafford-Fraser. The trojan room coffee pot, 1995. [Online; accessed 19-July-2016].

Robert Stephens. A survey of stream processing. Acta Informatica, 34(7): 491-541, 1997.

Michael Stonebraker, Uğur Cetintemel, and Stan Zdonik. The 8 requirements of real-time stream processing. ACM SIGMOD Record, 34(4):42-47, 2005.

L. M. Straker, C. M. Pollock, and J. E. Mangharam. The effect of shoulder posture on performance, discomfort and muscle fatigue whilst working on a visual display unit. International Journal of Industrial Ergonomics, 20 (1):1-10, 1997.

Arjan Stuiver and Ben Mulder. Cardiovascular state changes in simulated work environments. Frontiers in Neuroscience, 8, 2014.

Yu Sun, Sijung Hu, Vicente Azorin-Peris, Roy Kalawsky, and Stephen Greenwald. Noncontact imaging photoplethysmography to effectively access pulse rate variability. Journal of Biomedical Optics, 18(6):061205-061205, 2012a.

Yu Sun, Charlotte Papin, Vicente Azorin-Peris, Roy Kalawsky, Stephen Greenwald, and Sijung Hu. Use of ambient light in remote photoplethysmographic systems: Comparison between a high-performance camera and a low-cost webcam. Journal of Biomedical Optics, 17(3):037005-1-03700510, 2012b.

Yu Sun, Charlotte Papin, Vicente Azorin-Peris, Roy Kalawsky, Stephen Greenwald, and Sijung Hu. Use of ambient light in remote photoplethysmographic systems: Comparison between a high-performance camera and a low-cost webcam. Journal of Biomedical Optics, 17(3):0370051-03700510, 2012c.

Jan G. Svec and Svante Granqvist. Guidelines for selecting microphones for human voice production research. American Journal of Speech-Language Pathology, 19(4):356-368, 2010. 
Joachim Taelman, Steven Vandeput, Elke Vlemincx, Arthur Spaepen, and Sabine Van Huffel. Instantaneous changes in heart rate regulation due to mental load in simulated office work. European Journal of Applied Physiology, 111(7):1497-1505, 2011.

Tatsuto Takeuchi, Théodore Puntous, Anup Tuladhar, Sanae Yoshimoto, and Aya Shirama. Estimation of mental effort in learning visual search by measuring pupil response. PloS ONE, 6(7):1-5, 2011.

L. Tarassenko, M. Villarroel, A. Guazzi, J. Jorge, D. A. Clifton, and C. Pugh. Non-contact video-based vital sign monitoring using ambient light and auto-regressive models. Physiological Measurement, 35(5):807, 2014.

Mika Tarvainen. Estimation methods for nonstationary biosignals. $\mathrm{PhD}$ thesis, Kuopion yliopisto, 2004.

Louis G Tassinary and John T. Cacioppo. The skeletomotor system: Surface electromyography. In John T. Cacioppo, Louis G. Tassinary, and Gary G. Berntson, editors, Handbook of Psychophysiology, pages 163-199. Cambridge University Press, New York, NY, 2nd edition, 2000.

Maria L. Thomas and Michael B. Russo. Neurocognitive monitors: Toward the prevention of cognitive performance decrements and catastrophic failures in the operational environment. Aviation, Space, and Environmental Medicine, 78(Supplement 1):B144-B152, 2007.

J. Tiihonen, M. Kajola, and R. Hari. Magnetic mu rhythm in man. Neuroscience, 32(3):793-800, 1989. ISSN 0306-4522.

Olli Tikkanen, Piia Haakana, Arto J Pesola, Keijo Häkkinen, Timo Rantalainen, Marko Havu, Teemu Pullinen, and Taija Finni. Muscle activity and inactivity periods during normal daily life. PloS ONE, 8(1):e52228, Jan. 2013. ISSN 1932-6203.

Simone Tognetti, Maurizio Garbarino, Andrea Tommaso Bonanno, Matteo Matteucci, and Andrea Bonarini. Enjoyment recognition from physiological data in a car racing game. In Proceedings of the 3rd International Workshop on Affective Interaction in Natural Environments, pages 3-8. ACM, 2010.

Fumiharu Togo and Masaya Takahashi. Heart rate variability in occupational health-a systematic review. Industrial Health, 47(6):589-602, 2009.

Satoru Tokuda, Goro Obinata, Evan Palmer, and Alex Chaparro. Estimation of mental workload using saccadic eye movements in a free-viewing task. In Engineering in Medicine and Biology Society, EMBC, 2011 Annual International Conference of the IEEE, pages 4523-4529. IEEE, 2011. 
Jari Torniainen, Benjamin Cowley, Andreas Henelius, Kristian Lukander, and Satu Pakarinen. Feasibility of an electrodermal activity ring prototype as a research tool. In 37th Annual International Conference of the IEEE Engineering in Medicine and Biology Society, Milano, Italy, 2015.

Leonard J. Trejo, Arthur F. Kramer, and Josh A. Arnold. Event-related potentials as indices of display-monitoring performance. Biological Psychology, 40(1-2):33-71, May 1995. ISSN 03010511.

Yi-Fang Tsai, Erik Viirre, Christopher Strychacz, Bradley Chase, and TzyyPing Jung. Task performance and eye activity: Predicting behavior relating to cognitive workload. Aviation, Space, and Environmental Medicine, 78 (Supplement 1):B176-B185, 2007.

M. Ullsperger and D. Y. von Cramon. Subprocesses of performance monitoring: A dissociation of error processing and response competition revealed by event-related fMRI and ERPs. NeuroImage, 14(6):1387-401, Dec. 2001. ISSN 1053-8119.

Wouter van den Hoogen, Karolien Poels, Wijnand IJsselsteijn, and Yvonne de Kort. Between Challenge and Defeat: Repeated Player-Death and Game Enjoyment. Media Psychology, 15(4):443-459, Oct. 2012. ISSN 1521-3269.

Marjolein D. van der Zwaag, Joris H. Janssen, and Joyce H. D. M. Westerink. Directing Physiology and Mood through Music: Validation of an Affective Music Player. IEEE Transactions on Affective Computing, 4(1):57-68, Jan. 2013. ISSN 1949-3045.

Rik van Dinteren, Martijn Arns, Marijtje L. A. Jongsma, and Roy P. C. Kessels. P300 Development across the Lifespan: A Systematic Review and Meta-Analysis. PLoS ONE, 9(2), Feb. 2014. ISSN 1932-6203.

Marieke van Dooren, J. J. G. Gert-Jan de Vries, and Joris H. Janssen. Emotional sweating across the body: Comparing 16 different skin conductance measurement locations. Physiology \& Behavior, 106(2):298-304, May 2012. ISSN 1873-507X.

Arie M. Van Roon, Lambertus J. M. Mulder, Monika Althaus, and Gijsbertus Mulder. Introducing a baroreflex model for studying cardiovascular effects of mental workload. Psychophysiology, 41(6):961-981, 2004.

Francisco Varela, Jean-Philippe Lachaux, Eugenio Rodriguez, and Jacques Martinerie. The brainweb Phase synchronization and large-scale integration. Nature Reviews Neuroscience, 2(4):229-239, Apr. 2001. ISSN 1471003X. 
Olav Vassend and Stein Knardahl. Personality, affective response, and facial blood flow during brief cognitive tasks. International Journal of Psychophysiology, 55(3):265-278, 2005.

J. A. Veltman and A. W. K. Gaillard. Physiological workload reactions to increasing levels of task difficulty. Ergonomics, 41(5):656-669, 1998.

Dimitrios Ververidis and Constantine Kotropoulos. Emotional speech recognition: Resources, features, and methods. Speech Communication, 48(9): 1162-1181, 2006.

Henrica C. W. De Vet, Caroline B. Terwee, Lidwine B. Mokkink, and Dirk L. Knol. Systematic Reviews of Measurement Properties. In Measurement in Medicine: A Practical Guide, chapter 9, pages 275-315. Cambridge University Press, Cambridge, New York, 1st edition, 2011.

J. J. Vidal. Toward Direct Brain-Computer Communication. Annual Review of Biophysics and Bioengineering, 2(1):157-180, 1973.

Carmen Vidaurre, Nicole Krämer, Benjamin Blankertz, and Alois Schlögl. Time domain parameters as a feature for EEG-based Brain-Computer Interfaces. Neural Networks, 22(9):1313-1319, Nov. 2009. ISSN 0893-6080.

Alex Vincent, Fergus I. M. Craik, and John J. Furedy. Relations among memory performance, mental workload and cardiovascular responses. International Journal of Psychophysiology, 23(3):181-198, 1996.

Johannes Wagner, Jonghwa Kim, and Elisabeth André. From physiological signals to emotions: Implementing and comparing selected methods for feature extraction and classification. In Multimedia and Expo, 2005. ICME 2005. IEEE International Conference on, pages 940-943. IEEE, 2005.

Johannes Wagner, Elisabeth Andre, Florian Lingenfelser, and Jonghwa Kim. Exploring fusion methods for multimodal emotion recognition with missing data. Affective Computing, IEEE Transactions on, 2(4):206-218, 2011a.

Johannes Wagner, Florian Lingenfelser, and Elisabeth André. The social signal interpretation framework (ssi) for real time signal processing and recognition. In INTERSPEECH, pages 3245-3248, 2011b.

Qiang Wang and O. Sourina. Real-time mental arithmetic task recognition from EEG signals. IEEE Transactions on Neural Systems and Rehabilitation Engineering, 21(2):225-232, Mar. 2013. ISSN 1534-4320.

Ying Wang, Bryan Reimer, Jonathan Dobres, and Bruce Mehler. The sensitivity of different methodologies for characterizing drivers' gaze concentration under increased cognitive demand. Transportation Research Part F: Traffic Psychology and Behaviour, 26:227-237, 2014. 
Edmund Wascher, Holger Heppner, and Sven Hoffmann. Towards the measurement of event-related EEG activity in real-life working environments. International Journal of Psychophysiology: Official journal of the International Organization of Psychophysiology, 91(1):3-9, Jan. 2014. ISSN 18727697.

N. Watanabe, N. Hirai, T. Maehara, K. Kawai, H. Shimizu, F. Miwakeichi, and S. Uchida. The relationship between the visually evoked P300 eventrelated potential and gamma band oscillation in the human medial and basal temporal lobes. Neuroscience Research, 44(4):421-427, Dec. 2002. ISSN 01680102.

David Watson, Lee A. Clark, and Auke Tellegen. Development and validation of brief measures of positive and negative affect: The panas scales. Journal of Personality and Social Psychology, 54(6):1063, 1988.

Anna Weinberg, Huiting Liu, and Stewart A. Shankman. Blunted neural response to errors as a trait marker of melancholic depression. Biological Psychology, 113:100-107, 2016. ISSN 03010511.

K. H. Wesseling. Finapres, continuous noninvasive finger arterial pressure based on the method of Penaz. In Blood Pressure Measurements, pages 161-172. Springer, 1990.

Cornelis J. E. Wientjes. Respiration in psychophysiology: Methods and applications. Biological Psychology, 34(2):179-203, 1992.

Glenn F. Wilson. An analysis of mental workload in pilots during flight using multiple psychophysiological measures. The International Journal of Aviation Psychology, 12(1):3-18, 2002.

Glenn F. Wilson and Chris A. Russell. Operator functional state classification using multiple psychophysiological features in an air traffic control task. Human Factors: The Journal of the Human Factors and Ergonomics Society, 45(3):381-389, 2003a.

Glenn F. Wilson and Chris A. Russell. Operator functional state classification using multiple psychophysiological features in an air traffic control task. Human Factors: The Journal of the Human Factors and Ergonomics Society, 45(3):381-389, 2003b.

Glenn F. Wilson and Christopher A. Russell. Real-time assessment of mental workload using psychophysiological measures and artificial neural networks. Human Factors: The Journal of the Human Factors and Ergonomics Society, 45(4):635-644, 2003c. 
Glenn F. Wilson and Christopher A. Russell. Performance enhancement in an uninhabited air vehicle task using psychophysiologically determined adaptive aiding. Human Factors: The Journal of the Human Factors and Ergonomics Society, 49(6):1005-1018, 2007.

Nicola Wright and Amanda McGown. Vigilance on the civil flight deck: Incidence of sleepiness and sleep during long-haul flights and associated changes in physiological parameters. Ergonomics, 44(1):82-106, 2001.

Dongrui Wu, Christopher G. Courtney, Brent J. Lance, Shrikanth S. Narayanan, Michael E. Dawson, Kelvin S. Oie, and Thomas D. Parsons. Optimal arousal identification and classification for affective computing using physiological signals: Virtual reality stroop task. Affective Computing, IEEE Transactions on, 1(2):109-118, 2010.

Georgios N. Yannakakis, Héctor P. Martínez, and Arnav Jhala. Towards affective camera control in games. User Model User-Adap Inter, 20(4):313340, 2010.

K. Yashima, T. Sasaki, Y. Kageyama, M. Odagaki, and H. Hosaka. Application of wavelet analysis to the plethysmogram for the evaluation of mental stress. In Engineering in Medicine and Biology Society, 2005. IEEE-EMBS 2005. 27th Annual International Conference of the, pages 2781-2784. IEEE, 2006.

Fumihiko Yasuma and Jun ichiro Hayano. Respiratory sinus arrhythmia: Why does the heartbeat synchronize with respiratory rhythm? Chest, 125 (2):683-690, 2004. ISSN 0012-3692.

M. Yeasin, B. Bullot, and R. Sharma. Recognition of facial expressions and measurement of levels of interest from video. Multimedia, IEEE Transactions on, 8(3):500-508, June 2006. ISSN 1520-9210.

Kil-sang Yoo and Won-Hyung Lee. Mental stress assessment based on pulse photoplethysmography. In Consumer Electronics (ISCE), 2011 IEEE 15th International Symposium on, pages 323-326. IEEE, 2011.

Ruud Zaalberg, Antony Manstead, and Agneta Fischer. Relations between emotions, display rules, social motives, and facial behaviour. Cognition 8 Emotion, 18(2):183-207, Feb. 2004. ISSN 0269-9931.

M. Zecca, Silvestro Micera, M. C. Carrozza, and P. Dario. Control of Multifunctional Prosthetic Hands by Processing the Electromyographic Signal. Critical Reviews in Biomedical Engineering, 30(4-6):459-485, 2002. ISSN 0278-940X. 
Z. Zeng, M. Pantic, G. I. Roisman, and T. S. Huang. A survey of affect recognition methods: Audio, visual, and spontaneous expressions. IEEE Transactions on Pattern Analysis and Machine Intelligence, 31(1):39-58, 2009.

J. Zhai and A. Barreto. Stress detection in computer users through noninvasive monitoring of physiological signals. Biomedical Sciences Instrumentation, 42:495-500, 2006.

Xu Zhang, Xiang Chen, Wen-hui Wang, Ji-hai Yang, Vuokko Lantz, and Kongqiao Wang. Hand Gesture Recognition and Virtual Game Control Based on 3D Accelerometer and EMG Sensors. In Proceedings of the 14th International Conference on Intelligent User Interfaces, IUI '09, pages 401-406, New York, NY, USA, 2009. ACM. ISBN 978-1-60558-168-2.

Wenyi Zhao, Rama Chellappa, P. Jonathon Phillips, and Azriel Rosenfeld. Face recognition: A literature survey. ACM Computing Surveys (CSUR), 35(4):399-458, 2003.

Guojun Zhou, John H. L. Hansen, and James F. Kaiser. Nonlinear feature based classification of speech under stress. Speech and Audio Processing, IEEE Transactions on, 9(3):201-216, 2001. 